\title{
Renormalization of mixing angles
}

\section{Ansgar Denner, ${ }^{a}$ Stefan Dittmaier ${ }^{b}$ and Jean-Nicolas Lang ${ }^{c}$}

${ }^{a}$ Universität Würzburg, Institut für Theoretische Physik und Astrophysik, 97074 Würzburg, Germany

${ }^{b}$ Albert-Ludwigs-Universität Freiburg, Physikalisches Institut, 79104 Freiburg, Germany

${ }^{c}$ Universität Zürich, Physik-Institut, CH-8057 Zürich, Switzerland

E-mail: ansgar.denner@physik.uni-wuerzburg.de, stefan.dittmaier@physik.uni-freiburg.de, jlang@physik.uzh.ch

ABSTRACT: We discuss the renormalization of mixing angles for theories with extended scalar sectors. Motivated by shortcomings of existing schemes for mixing angles, we review existing renormalization schemes and introduce new ones based on on-shell conditions or symmetry requirements such as rigid or background-field gauge invariance. Considering in particular the renormalization of the mixing angles in the Two-Higgs-Doublet Model and the Higgs-Singlet Extension of the Standard Model, we compare electroweak corrections within these models for a selection of renormalization schemes. As specific examples, we present next-to-leading-order results on the four-fermion decays of heavy and light CPeven Higgs bosons, $\mathrm{H}_{1} / \mathrm{H}_{2} \rightarrow \mathrm{WW} / \mathrm{ZZ} \rightarrow 4 f$, and on electroweak Higgs-boson production processes, i.e. Higgs-strahlung and vector-boson fusion. We find that our new proposals for on-shell and symmetry-based renormalization conditions are well-behaved for the considered benchmark scenarios in both models.

KEYwords: NLO Computations, Phenomenological Models

ARXiv EPRINT: 1808.03466 


\section{Contents}

1 Introduction 1

2 Preliminaries 3

2.1 Renormalization transformation for mixing fields 3

2.2 Higgs-singlet extension of the Standard Model 5

$\begin{array}{lll}2.3 & \text { Two-Higgs-doublet model } & 6\end{array}$

3 Renormalization schemes for mixing matrices $\quad 7$

3.1 Renormalization of mixing angles in $\overline{\mathrm{MS}}$ schemes 8

3.2 Physical (on-shell) renormalization conditions for mixing angles 9

3.2.1 Higgs-singlet extension of the Standard Model 11

$\begin{array}{lll}3.2 .2 & \text { Two-Higgs-doublet model } & 13\end{array}$

$\begin{array}{ll}3.3 & \text { Renormalization of mixing angles based on symmetries } \\ & 16\end{array}$

3.3.1 Rigid symmetry and wave-function renormalization for physical states 16

3.3.2 Rigid symmetry and wave-function renormalization for unphysical $\begin{array}{ll}\text { states } & 19\end{array}$

\begin{tabular}{ll} 
3.3.3 & Background-field gauge invariance \\
\hline
\end{tabular}

$\begin{array}{ll}3.4 & \text { Summary of renormalization schemes } \\ 3.5 & \text { Paramer }\end{array}$

3.5 Parameter conversion between renormalization schemes 25

3.5.1 Matching procedure and running couplings 25

$\begin{array}{ll}\text { 3.5.2 Higher-order ambiguities in the conversion } & 27\end{array}$

4 Phenomenological results $\quad 28$

4.1 Higgs-boson decays $\mathrm{H}_{1,2} \rightarrow \mathrm{WW} / \mathrm{ZZ} \rightarrow 4$ fermions 28

4.1.1 Higgs-singlet extension of the Standard Model 28

$\begin{array}{lll}\text { 4.1.2 Two-Higgs-doublet model } & 31\end{array}$

4.2 Higgs-boson production processes at the LHC 35

$\begin{array}{lll}\text { 4.2.1 Cut setup and parameters } & 35\end{array}$

4.2.2 Higgs-strahlung in the HSESM 36

4.2.3 Higgs-strahlung in the THDM 38

4.2.4 Higgs production via vector-boson fusion in the THDM 41

5 Conclusions $\quad 42$

A Translation of conventions for the HSESM 44

B Translation of self-energies to the background-field method 44

B.1 Higgs sector of the HSESM $\quad 45$

$\begin{array}{lll}\text { B.2 Higgs sector of THDM } & 45\end{array}$ 
C Tadpole contributions to scalar mixing and self-energies

C.1 Tadpole counterterms in the FJTS 47

C.2 Tadpole counterterms in the PRTS 48

$\begin{array}{ll}\text { D Vertex corrections for on-shell schemes } & 48\end{array}$

E Background-field Ward identities $\quad \mathbf{5 0}$

E.1 A Standard Model example 51

$\begin{array}{lll}\text { E.2 A THDM example } & 54\end{array}$

F Parameter conversion tables $\quad \mathbf{5 5}$

F.1 HSESM scenarios of table $3 \quad 55$

F.2 THDM scenarios of table $6 \quad 56$

\section{Introduction}

After the discovery of a Higgs boson at the Large Hadron Collider (LHC) [1, 2], the investigation of the Higgs sector is still of prime importance for particle physics. Theories with extended Higgs sectors typically contain additional scalar multiplets leading to physical scalar states that mix. Simple examples of such extensions are the Two-HiggsDoublet Model (THDM) [3, 4] and the Higgs-Singlet Extension of the Standard Model (HSESM) [5-7]. For a precise study of such theories, next-to-leading-order (NLO) QCD and electroweak (EW) corrections have to be taken into account. This requires a renormalization of these models and thus the renormalization of mixing angles or, more generally, of mixing matrices.

The need for renormalization of mixing matrices already appears in the Standard Model (SM) where the quark-mixing matrix has to be renormalized. While this is phenomenologically unimportant owing to the smallness of the down-type quark masses, the problem has nevertheless found quite some interest in the literature, and the corresponding theoretical developments have also influenced the work on the renormalization of mixing matrices in scalar sectors, which is the subject of this paper. A first renormalization condition for the quark-mixing matrix based on on-shell field-renormalization constants of the quark fields was proposed in refs. $[8,9]$. This prescription is simple, symmetric in the fields that mix, and smoothly connected to the limit of degenerate quark masses. Later it was discovered [10] that the straightforward use of the renormalization condition of refs. [8, 9] gives rise to gauge-parameter-dependent counterterms for the quark-mixing matrix and thus to a gauge-parameter-dependent parametrization of $S$-matrix elements in terms of renormalized parameters. In the sequel, various proposals were made for a gauge-parameter-independent renormalization of the quark-mixing matrix [10-15]. Typically, these are cumbersome to apply, their generalization beyond one-loop order remains unclear, and/or they potentially lead to singularities in the $S$-matrix elements for degenerate quark masses. The last 
problem occurs, in particular, in the modified minimal subtraction $(\overline{\mathrm{MS}})$ scheme. A gaugeindependent, symmetric, physical renormalization condition was proposed in ref. [16]. It was also suggested to define the quark-mixing matrix counterterm from the quark-field renormalization constants calculated in the 't Hooft-Feynman gauge [17]. Generalizing this idea, it was argued in ref. [12] that any renormalization scheme for the quark-mixing matrix may be viewed as a gauge-invariant scheme by definition, in the sense that $S$-matrix elements remain invariant if the gauge used in the calculation of the loop corrections and all other renormalization constants is changed, while keeping the defining gauge for the renormalization constants of the quark-mixing matrix fixed.

The need for suitable renormalization schemes for mixing angles becomes more important in extensions of the SM. Specific examples are models with additional Higgs bosons, additional vector bosons, or additional fermions. In particular, for the renormalization of mixing angles in the scalar sector a variety of schemes were used in the literature. Specifically, the renormalization of the mixing angle $\beta$ in the Minimal Supersymmetric Standard Model (MSSM) was discussed in refs. [18-22].

The renormalization of the mixing angles $\alpha$ and $\beta$ in the THDM was considered in refs. [23-29]. Kanemura and collaborators [23, 25, 30] used the vanishing of the renormalized non-diagonal "on-shell" scalar 2-point functions to fix the mixing-angle renormalization. ${ }^{1}$ Despite the choice of "on-shell" momenta these conditions do not derive from $S$-matrix elements and are gauge dependent. In ref. [24], the mixing angle $\alpha$ was fixed by the condition that the mixing self-energy of the CP-even Higgs bosons vanishes "on-shell", while $\beta$ was renormalized requiring that the ratio of vacuum expectation values (vevs), $v_{2} / v_{1}$, is expressed in terms of the "true" vacua following the treatment of refs. $[18,19]$ in the MSSM. The authors of ref. [26] employ the renormalization conditions of ref. [23] within the FJ Tadpole Scheme by Fleischer and Jegerlehner [31] and define gauge-independent counterterms based on the "pinch-technique" prescription [32]. ${ }^{2}$

In the series of papers $[27,28]$ the gauge dependence of the $\overline{\mathrm{MS}}$ definition of mixing angles with respect to different tadpole counterterm schemes was investigated, and predictions were compared against before-mentioned schemes based on mixing energies. Finally, in ref. [29] new (gauge-independent) $\overline{\mathrm{MS}}$ schemes were introduced, replacing a mixing-angle definition by the $\overline{\mathrm{MS}}$ renormalization of a coupling parameter of the Higgs potential.

For the HSESM the mixing-angle renormalization was discussed in refs. [28, 35-37]. In ref. [35], the renormalization scheme of ref. [23] was transferred to the HSESM. The authors of ref. [36] discuss different renormalization schemes based on conditions on the scalar mixing energy or $\overline{\mathrm{MS}}$ renormalization. While in ref. [28] an $\overline{\mathrm{MS}}$ scheme was compared with schemes based on on-shell self-energies, in ref. [37] different $\overline{\mathrm{MS}}$ schemes were studied.

\footnotetext{
${ }^{1}$ We put "on-shell" in quotation marks here, since we want to reserve this word to conditions that are based on $S$-matrix elements rather than simply taking momenta on their mass shell in more general quantities such as Green functions, self-energies, etc..

${ }^{2}$ Following the arguments of refs. [33, 34] we consider the "pinch technique" just as one of many physically equivalent choices to fix the gauge arbitrariness in off-shell quantities (related to the 't Hooft-Feynman gauge of the quantum fields in the background-field method) rather than singling out "its gauge-invariant part" in any sense.
} 
The purpose of this paper is a discussion of renormalization prescriptions and schemes for mixing angles in general scalar sectors of gauge theories and a comparison of different schemes in concrete phenomenological applications in the THDM and the HSESM. We critically review existing renormalization prescriptions and introduce new ones that exhibit several desirable properties [20]. In particular, we introduce genuine on-shell renormalization conditions for mixing angles based on combinations of suitable $S$-matrix elements and put renormalization schemes based on symmetry requirements such as rigid invariance or background-field gauge invariance on a general footing.

This paper is organized as follows. In section 2 we specify some useful definitions and conventions. In section 3 we review and introduce renormalization schemes for mixing angles in scalar sectors. We begin with a discussion of existing $\overline{\mathrm{MS}}$ renormalization schemes, followed by sections where we construct new on-shell renormalization schemes and renormalization schemes based on symmetries. In section 4 we provide a numerical discussion of renormalization schemes in applications to Higgs decays into 4 fermions as well as Higgs production at the LHC via Higgs-strahlung or weak vector-boson fusion. After the conclusion in section 5, we give translation rules of our conventions to other formulations in the literature in appendix A. Further appendices provide explicit analytical results for quantities used in the various renormalization schemes, including scalar self-energies in the background-field method, the tadpole contributions to the scalar self-energies, vertex corrections for on-shell schemes, and a discussion of background-field Ward identities in different tadpole counterterm schemes.

\section{Preliminaries}

\subsection{Renormalization transformation for mixing fields}

As specific examples for the renormalization of mixing angles we consider the mixing of scalar fields in the THDM and the HSESM. Both theories involve two physical CP-even scalar bosons. Let the corresponding fields in the symmetric basis be $\eta_{1}$ and $\eta_{2}$ and the fields in the physical mass-eigenstate basis $H_{1}$ and $H_{2}$. The fields are related via a rotation

$$
\boldsymbol{\eta}=\left(\begin{array}{l}
\eta_{1} \\
\eta_{2}
\end{array}\right)=R(\alpha)\left(\begin{array}{l}
H_{1} \\
H_{2}
\end{array}\right)=R(\alpha) \mathbf{H}, \quad R(\alpha)=\left(\begin{array}{cc}
c_{\alpha} & -s_{\alpha} \\
s_{\alpha} & c_{\alpha}
\end{array}\right),
$$

where we use the shorthand notations $c_{\alpha}=\cos \alpha$ and $s_{\alpha}=\sin \alpha$. In the general case of more than two mixing fields $H_{i}$, the matrix $R(\boldsymbol{\alpha})$ depends on a set of mixing angles $\boldsymbol{\alpha}=\left\{\alpha_{i}\right\}$.

Performing the renormalization in the physical basis in the complete on-shell scheme $[9$, 38], the renormalization transformations for the mixing angle and the field renormalization constants of the scalar fields read

$$
\begin{aligned}
\alpha_{\mathrm{B}} & =\alpha+\delta \alpha, \\
\mathbf{H}_{\mathrm{B}} & =\left(Z^{H}\right)^{1 / 2} \mathbf{H}
\end{aligned}
$$


with

$$
\left(Z^{H}\right)^{1 / 2}=\left(\begin{array}{cc}
\left(Z^{H}\right)_{11}^{1 / 2} & \left(Z^{H}\right)_{12}^{1 / 2} \\
\left(Z^{H}\right)_{21}^{1 / 2} & \left(Z^{H}\right)_{22}^{1 / 2}
\end{array}\right)=\mathbf{1}+\frac{1}{2} \delta Z^{H}=\left(\begin{array}{cc}
1+\frac{1}{2} \delta Z_{11}^{H} & \frac{1}{2} \delta Z_{12}^{H} \\
\frac{1}{2} \delta Z_{21}^{H} & 1+\frac{1}{2} \delta Z_{22}^{H}
\end{array}\right),
$$

where bare quantities carry an index $\mathrm{B}$, and $\delta \alpha$ and $\delta Z_{i j}^{H}$ represent the renormalization constants for the mixing angle $\alpha$ and the scalar fields corresponding to mass eigenstates. In the complete on-shell scheme of refs. [9, 38], the non-diagonal field-renormalization constants are fixed as

$$
\delta Z_{i j}^{H}=\frac{2}{M_{\mathrm{H}_{i}}^{2}-M_{\mathrm{H}_{j}}^{2}} \Sigma_{i j}\left(M_{\mathrm{H}_{j}}^{2}\right), \quad i \neq j
$$

where $M_{\mathrm{H}_{i}}$ and $M_{\mathrm{H}_{j}}$ denote the masses of the corresponding scalar bosons $\mathrm{H}_{i}$ and $\mathrm{H}_{j}$, and $\Sigma_{i j}$ their mixing energy. In this paper we consistently use the convention that the self and mixing energies include explicit and implicit tadpole contributions, i.e. they are defined as the higher-order contributions to the inverse propagators which at one-loop order can be depicted as

$$
\Sigma_{i j}=i \longrightarrow \text { (1) } j+i \longrightarrow j+i \longrightarrow \text { (1) } j \text {. }
$$

The first contribution is the bare loop one-particle-irreducible (1PI) energy and the second term the corresponding 2-point tadpole counterterm. The third and forth terms are the explicit tadpole loop and tadpole counterterm contributions, respectively. Note that no counterterms other than the ones from the tadpoles (such as mass or field renormalization constants) are included in $\Sigma_{i j}$. In the renormalization of the SM Higgs sector described in refs. [9, 34], the vev $v$ is renormalized in such a way that the Higgs field has vanishing vev, and consequently, all explicit tadpole contributions vanish, i.e. the third and forth term in (2.6) add up to zero. However, implicit tadpole counterterms may remain in the second term, but their explicit form depends on the considered fields and on the tadpole counterterm scheme in use. If we use self-energies without any tadpole contributions, we will indicate this with an extra index 1PI for one-particle irreducible. If not stated otherwise, the formulas are given in the Fleischer and Jegerlehner tadpole counterterm scheme as used in ref. [27] for which the construction of the implicit tadpole contributions to 2-point functions is given in appendix C. This convention for the tadpole contributions in $\Sigma_{i j}$ is in agreement with the one of refs. [31, 39].

In the presence of mixing, the well-known problem of degenerate states in time-independent perturbation theory of quantum mechanics also appears in quantum field theory and becomes first apparent in the one-loop renormalization. The condition (2.5) shifts corrections from the mixing of states that are induced by external (non-diagonal) self-energy insertions to vertex counterterms. For degenerate masses, $M_{\mathrm{H}_{i}} \rightarrow M_{\mathrm{H}_{j}}$, the constants $\delta Z_{i j}^{H}$ become singular, and thus also the $S$-matrix elements with external $H_{i}, H_{j}$ fields unless this singularity is cancelled by some other contribution. As the loop diagrams are regular in this limit, the cancellation should come from another counterterm. This is where the 
mixing angles come into play, and one expects that an appropriate renormalization of the mixing angles can make the $S$-matrix well-behaved as we will sketch in the following. ${ }^{3}$

Combining (2.1) for bare fields with (2.4) and using

$$
R\left(\boldsymbol{\alpha}_{\mathrm{B}}\right)=R(\boldsymbol{\alpha})+\delta R(\boldsymbol{\alpha}, \delta \boldsymbol{\alpha})
$$

yields to one-loop accuracy

$$
\boldsymbol{\eta}_{\mathrm{B}}=R(\boldsymbol{\alpha})\left(1+R^{\mathrm{T}}(\boldsymbol{\alpha}) \delta R(\boldsymbol{\alpha}, \delta \boldsymbol{\alpha})+\frac{1}{2} \delta Z^{H}\right) \mathbf{H} .
$$

Thus, if the mixing matrix $R$ in the Lagrangian results only from the rotation between symmetric and mass eigenstates as in (2.1), the counterterm to the mixing matrix appears only in the combination

$$
R^{\mathrm{T}}(\boldsymbol{\alpha}) \delta R(\boldsymbol{\alpha}, \delta \boldsymbol{\alpha})+\frac{1}{2} \delta Z^{H}
$$

in $S$-matrix elements. For the specific case of a single mixing angle this gives rise to the two combinations

$$
-\delta \alpha+\frac{1}{2} \delta Z_{12}^{H}, \quad \delta \alpha+\frac{1}{2} \delta Z_{21}^{H}
$$

Choosing the mixing-angle counterterm appropriately allows one to cancel the singularity for degenerate masses in $S$-matrix elements arising from the denominators in (2.5).

If the mixing angle $\alpha$ is promoted to a physical parameter upon eliminating some bare parameter of the Lagrangian in its favour, the counterterm $\delta \alpha$ appears also independently of $\delta Z_{12 / 21}^{H}$ in $S$-matrix elements. This is the case in the THDM and the HSESM if a quartic coupling parameter $\lambda_{i}$ of the Higgs potential is eliminated in favour of the mixing angle $\alpha$ in the sector of CP-even Higgs bosons. However, it turns out that in this case the mixingangle counterterm appears in addition to (2.10) only in the combination $\left(M_{\mathrm{H}_{1}}^{2}-M_{\mathrm{H}_{2}}^{2}\right) \delta \alpha$. This can easily be verified by considering the coupling parameter $\lambda_{i}$ as a function of Higgs masses and mixing angles (see, for instance, eq. (3.10) in ref. [27] or eq. (2.20) in ref. [29]). This statement can be generalized to mixing angles in more general theories. Note, however, that the situation is different for mixing angles defined via vevs, such as $\beta$ in the THDM or the MSSM.

\subsection{Higgs-singlet extension of the Standard Model}

In the HSESM we associate $\eta_{2}$ in eq. (2.1) with the scalar component of the Higgs doublet

$$
\Phi=\left(\begin{array}{c}
\phi^{+} \\
\frac{1}{\sqrt{2}}\left(v_{2}+\eta_{2}+\mathrm{i} \chi\right)
\end{array}\right),
$$

where $v_{2}$ is the corresponding vev and $\phi^{+}, \chi$ the would-be Goldstone-boson fields. The field $\eta_{1}$ is the field excitation of the (canonically normalized) Higgs singlet field

$$
\sigma=v_{1}+\eta_{1}
$$

\footnotetext{
${ }^{3}$ In analogy to time-independent perturbation theory of quantum mechanics, the freedom of renormalization of the mixing angles corresponds to the freedom in choosing a basis for the (quasi) degenerate subsystem.
} 
which acquires the vev $v_{1}$. The Higgs potential of the considered variant of the HSESM is given by $[5-7,40]$

$$
V_{\mathrm{HSESM}}=-\mu_{2}^{2} \Phi^{\dagger} \Phi-\frac{1}{2} \mu_{1}^{2} \sigma^{2}+\frac{\lambda_{2}}{4}\left(\Phi^{\dagger} \Phi\right)^{2}+\frac{\lambda_{1}}{16} \sigma^{4}+\frac{\lambda_{3}}{2} \Phi^{\dagger} \Phi \sigma^{2},
$$

which possesses a $\mathbb{Z}_{2}$ symmetry under $\sigma \rightarrow-\sigma$. Translation rules of these conventions to the ones used in refs. $\left[28,37\right.$ are given in appendix A. The masses $M_{\mathrm{W}}$ and $M_{\mathrm{Z}}$ of the weak gauge bosons are given by

$$
M_{\mathrm{W}}=\frac{1}{2} g_{2} v_{2}, \quad M_{\mathrm{Z}}=\frac{1}{2} \sqrt{g_{2}^{2}+g_{1}^{2}} v_{2},
$$

where $g_{2}$ and $g_{1}$ are the $\mathrm{SU}(2)$ and $\mathrm{U}(1)$ gauge couplings, respectively.

\section{$2.3 \quad$ Two-Higgs-doublet model}

In the THDM, $\eta_{1}$ and $\eta_{2}$ are the neutral CP-even scalar components of the two Higgsdoublet fields

$$
\Phi_{i}=\left(\begin{array}{c}
\phi_{i}^{+} \\
\frac{1}{\sqrt{2}}\left(v_{i}+\eta_{i}+\mathrm{i} \chi_{i}\right)
\end{array}\right), \quad i=1,2,
$$

and we associate the mass eigenstate $\mathrm{H}_{1}=\mathrm{H}_{\mathrm{h}}=\mathrm{H}$ with the heavy scalar and $\mathrm{H}_{2}=\mathrm{H}_{\mathrm{l}}=\mathrm{h}$ with the light one. The self-interaction of the two Higgs doublets is induced by the Higgs potential $[3,4]^{4}$

$$
\begin{aligned}
V_{\mathrm{THDM}}= & m_{1}^{2} \Phi_{1}^{\dagger} \Phi_{1}+m_{2}^{2} \Phi_{2}^{\dagger} \Phi_{2}-m_{12}^{2}\left(\Phi_{1}^{\dagger} \Phi_{2}+\Phi_{2}^{\dagger} \Phi_{1}\right) \\
& +\frac{\lambda_{1}}{2}\left(\Phi_{1}^{\dagger} \Phi_{1}\right)^{2}+\frac{\lambda_{2}}{2}\left(\Phi_{2}^{\dagger} \Phi_{2}\right)^{2}+\lambda_{3}\left(\Phi_{1}^{\dagger} \Phi_{1}\right)\left(\Phi_{2}^{\dagger} \Phi_{2}\right)+\lambda_{4}\left(\Phi_{1}^{\dagger} \Phi_{2}\right)\left(\Phi_{2}^{\dagger} \Phi_{1}\right) \\
& +\frac{\lambda_{5}}{2}\left[\left(\Phi_{1}^{\dagger} \Phi_{2}\right)^{2}+\left(\Phi_{2}^{\dagger} \Phi_{1}\right)^{2}\right]
\end{aligned}
$$

which has a $\mathbb{Z}_{2}$ symmetry w.r.t. $\Phi_{1} \rightarrow-\Phi_{1}$ and $\Phi_{2} \rightarrow \Phi_{2}$ that is only softly broken by the $m_{12}^{2}$ term.

The two scalar doublets in the THDM contain, in addition to the scalar fields $\eta_{i}$, also pseudoscalar $\chi_{i}$ and charged scalar $\phi_{i}^{+}$fields. These are transformed to the physical basis as follows,

$$
\left(\begin{array}{c}
\phi_{1}^{ \pm} \\
\phi_{2}^{ \pm}
\end{array}\right)=R(\beta)\left(\begin{array}{c}
G^{ \pm} \\
H^{ \pm}
\end{array}\right), \quad\left(\begin{array}{l}
\chi_{1} \\
\chi_{2}
\end{array}\right)=R(\beta)\left(\begin{array}{c}
G_{0} \\
A_{0}
\end{array}\right) \quad \text { with } \quad R(\beta)=\left(\begin{array}{cc}
c_{\beta} & -s_{\beta} \\
s_{\beta} & c_{\beta}
\end{array}\right) .
$$

Here $G^{ \pm}$and $G_{0}$ are the charged and neutral would-be Goldstone-boson fields, and $H^{ \pm}$ and $A_{0}$ the physical charged and pseudoscalar Higgs fields, respectively. The mixing angle $\beta$ is related to the vevs $v_{i}$ of the two scalar doublets via $t_{\beta} \equiv \tan \beta=v_{2} / v_{1}$, and we use

\footnotetext{
${ }^{4}$ For more details on this model, its parametrization and renormalization consider also refs. [28, 29], which follow the conventions of the original references.
} 
the abbreviations $c_{\beta}=\cos \beta$ and $s_{\beta}=\sin \beta$. The masses $M_{\mathrm{W}}$ and $M_{\mathrm{Z}}$ of the weak gauge bosons are given by

$$
M_{\mathrm{W}}=\frac{1}{2} g_{2} v, \quad M_{\mathrm{Z}}=\frac{1}{2} \sqrt{g_{2}^{2}+g_{1}^{2}} v, \quad v=\sqrt{v_{1}^{2}+v_{2}^{2}} .
$$

The renormalization transformations for the mixing angle $\beta$ and the pseudoscalar fields in the complete on-shell scheme can be written as

$$
\begin{aligned}
\beta_{\mathrm{B}} & =\beta+\delta \beta \\
\left(\begin{array}{c}
G_{0 \mathrm{~B}} \\
A_{0 \mathrm{~B}}
\end{array}\right) & =\left(\begin{array}{cc}
1+\frac{1}{2} \delta Z_{G_{0} G_{0}} & \frac{1}{2} \delta Z_{G_{0} A_{0}} \\
\frac{1}{2} \delta Z_{A_{0} G_{0}} & 1+\frac{1}{2} \delta Z_{A_{0} A_{0}}
\end{array}\right)\left(\begin{array}{c}
G_{0} \\
A_{0}
\end{array}\right),
\end{aligned}
$$

where $\delta \beta$ and $\delta Z_{\ldots}$ represent the renormalization constants for the mixing angle $\beta$ and the physical pseudoscalar $A_{0}$ and the would-be-Goldstone field $G_{0}$. Similar equations can be written for the charged scalar fields.

\section{Renormalization schemes for mixing matrices}

In this section we review existing renormalization schemes for mixing angles and propose and discuss new ones. In ref. [20] desirable properties for the renormalization of mixing matrices were formulated:

- The mixing-angle renormalization should be gauge independent, i.e. renormalized $S$ matrix elements should be gauge-independent functions of the renormalized mixing angles.

- The mixing-angle renormalization should be symmetric with respect to the mixing degrees of freedom. Moreover, the renormalized mixing angle should be independent of a specific physical process.

- The mixing-angle renormalization should not spoil the numerical stability of the perturbative expansion; in particular, the running of parameters and radiative corrections to physical observables should be accessible via perturbation theory.

We add a further condition, which could be viewed as a refinement of the third condition of ref. [20]:

- In the limit of degenerate masses of the mixing particles or in the limit of extreme mixing angles, no singularities should be introduced in physical observables, i.e. $S$ matrix elements should behave smoothly in these limits. Furthermore, there should be no "dead corners" in the parameter space of the model where a renormalized input parameter nominally goes to infinity. ${ }^{5}$

\footnotetext{
${ }^{5}$ See, e.g., the discussion of the $\overline{\mathrm{MS}}\left(\lambda_{3}\right)$ and $\mathrm{FJ}\left(\lambda_{3}\right)$ schemes of the THDM in refs. [29, 41], where the parametrization of the mixing angle $\alpha$ by the coupling $\lambda_{3}$ develops a singularity for $\cos (2 \alpha) \rightarrow 0$.
} 
Focusing on these requirements, as far as possible, we propose new renormalization schemes, specifically for, but not limited to the THDM and the HSESM, and compare NLO predictions for some processes obtained with some old and the new schemes.

We write the relations between renormalization constants and self-energies without taking real parts leading to renormalization constants with imaginary parts. This is appropriate for the complex-mass scheme [42, 43]. In the usual on-shell scheme, the real part of the self-energies should be taken in all renormalization conditions.

\subsection{Renormalization of mixing angles in $\overline{\mathrm{MS}}$ schemes}

A straightforward, universal renormalization scheme, which does not distinguish a specific mass scale in the case of the renormalization of a mixing angle, is provided by $\overline{\mathrm{MS}}$ renormalization, where the renormalization constants contain only ultraviolet(UV)-divergent parts along with some universal finite constants, i.e. the combination

$$
\Delta=\frac{2}{4-D}-\gamma_{\mathrm{E}}+\ln (4 \pi)
$$

in dimensional regularization, where $D$ is the space-time dimension and $\gamma_{\mathrm{E}}$ the EulerMascheroni constant. $\overline{\mathrm{MS}}$ renormalization can be straightforwardly applied to arbitrary mixing matrices.

The $\overline{\mathrm{MS}}$ renormalization of mixing angles is, by construction, symmetric in the fields that mix and does not depend on a specific observable. Since mixing angles are defined in the physical basis, their $\overline{\mathrm{MS}}$ renormalization depends on the precise treatment of tadpoles $[27,31]$. If tadpoles are treated in a conventional way, i.e. if renormalized tadpoles are set to zero in the course of parameter renormalization and tadpole counterterms partially absorbed into bare masses (see, e.g., the renormalization of the SM in refs. [9, 39]), the counterterms for the mixing angles become gauge dependent. The tadpole scheme based on ref. [9] where the bare masses are defined as the coefficients of the terms quadratic in the physical fields in the Lagrangian is referred to as PRTS (Parameter-Renormalized Tadpole Scheme) in the following. ${ }^{6}$ We denote the $\overline{\mathrm{MS}}$ scheme applied to mixing angles based on the PRTS as $\overline{\mathrm{MS}}$ (PRTS) in the following. If tadpoles are treated in the FJ Tadpole Scheme (FJTS) [26, 27, 31], i.e. removed by a suitable field redefinition, the resulting $\overline{\mathrm{MS}}$ renormalization scheme applied to mixing angles, denoted in the following as $\overline{\mathrm{MS}}$ (FJTS), is by construction gauge independent. The $\overline{\mathrm{MS}}$ (PRTS) and $\overline{\mathrm{MS}}$ (FJTS) schemes have been worked out for the THDM and the HSESM in different variants in refs. [26-29] and [28, 37], respectively. The "Tadpole scheme" for the renormalization of $\tan \beta$ in the Minimal Supersymmetric Standard Model studied in ref. [20] is equivalent to the MS(FJTS) scheme for the mixing angle $\beta$. It was found that this scheme leads to an unacceptably large scheme uncertainty in the renormalization of $\tan \beta$. In ref. [28] the $\overline{\mathrm{MS}}$ (FJTS) scheme has been applied to the renormalization of mixing angles $\alpha$ and $\beta$ in the THDM and the HSESM in an NLO study of heavy and light Higgs production in Higgs-strahlung, $\mathrm{pp} \rightarrow \mathrm{H}_{1,2} \mu^{-} \mu^{+}+X$, and vector-boson fusion, $\mathrm{pp} \rightarrow \mathrm{H}_{1,2 \mathrm{jj}}+X$. The results of the $\overline{\mathrm{MS}}$ (FJTS) scheme turned out

\footnotetext{
${ }^{6}$ This tadpole counterterm scheme differs from others by the fact that no implicit tadpole counterterms appear in 2-point functions with physical external fields.
} 
to be unstable and suffering from large scale uncertainties in many scenarios, while results in schemes based on on-shell self- and mixing energies remained well-behaved. NLO results obtained with the $\overline{\mathrm{MS}}$ (PRTS) and $\overline{\mathrm{MS}}$ (FJTS) schemes were compared in quite some detail for the light Higgs-boson decays $\mathrm{H}_{2} \rightarrow \mathrm{WW} / \mathrm{ZZ} \rightarrow 4$ fermions in the THDM $[29,41]$ and the HSESM [37]. While the results of the two schemes are perturbatively stable in the HSESM, with nice plateaus showing up in the variation of the renormalization scale at NLO, the results on $\mathrm{H}_{2} \rightarrow 4 f$ in the $\overline{\mathrm{MS}}$ (FJTS) scheme lead to serious perturbative instabilities in THDM scenarios that are away from the alignment limit, where $|\cos (\beta-\alpha)|$ is not small.

All $\overline{\mathrm{MS}}$ renormalization schemes for mixing angles give rise to large corrections in the limit of degenerate masses. This enhancement results from terms of the form (2.10) in $S$ matrix elements. In $\overline{\mathrm{MS}}$ schemes, $\delta \alpha$ cancels only the UV-divergent parts, but the remaining UV-finite terms in (2.10) resulting from the field (or wave-function) renormalization become singular for $M_{\mathrm{H}_{i}} \rightarrow M_{\mathrm{H}_{j}}$. The size of these terms, which are also present in the $\overline{\mathrm{MS}}$ (PRTS) scheme, is enhanced by additional tadpole contributions in the $\overline{\mathrm{MS}}$ (FJTS) scheme. While for $\tan \beta$ similar enhancements due to additional tadpole contributions take place, the limit $M_{\mathrm{H}_{i}} \rightarrow M_{\mathrm{H}_{j}}$ does not introduce singularities connected with the renormalization of $\beta$, so that no corresponding singular contributions to $S$-matrix elements can result. In this sense, the situation for true mixing angles (such as $\alpha$ ) is more involved.

Instead of imposing the $\overline{\mathrm{MS}}$ condition on the mixing angles, $\overline{\mathrm{MS}}$ renormalization can be applied directly to parameters of the Higgs potential. This idea was, e.g., pursued in the " $\lambda_{3}$ " schemes of refs. $[29,41]$ as an alternative to the $\overline{\mathrm{MS}}$ renormalization of the mixing angle $\alpha$ in the THDM; specifically, $\alpha$ was replaced as input parameter by the coupling $\lambda_{3}$, which was $\overline{\mathrm{MS}}$ renormalized. While such a renormalization condition is gauge independent and does not lead to singularities for degenerate masses, problematic regions ("dead corners") in the parameter space show up where the relation between the distinguished coupling and the mixing angle cannot be inverted. In the " $\lambda_{3}$ " schemes of refs. [29, 41], for instance, a singularity in the parametrization of observables by $\lambda_{3}$ occurs in scenarios in which $\cos (2 \alpha) \rightarrow 0$. To circumvent this problem in the THDM, one would have to patch the parameter space by switching from $\lambda_{3}$ to another scalar coupling as renormalized parameter.

In summary, $\overline{\mathrm{MS}}$ renormalization schemes for mixing angles have some desirable properties (simplicity, symmetry, process independence), but suffer, in general, from problems with perturbative stability in certain parameter regions, such as for mass degeneracy of the mixing fields. Moreover, care has to be taken in view of gauge dependence. On the other hand, it should be mentioned that $\overline{\mathrm{MS}}$ renormalization offers a simple way to estimate perturbative stability by varying the renormalization scale in predictions and checking for a stabilization of results in the transition from leading order (LO) to NLO.

\subsection{Physical (on-shell) renormalization conditions for mixing angles}

The renormalization of mixing angles can be directly fixed from observables or $S$-matrix elements that depend on these mixing angles at LO. Such on-shell renormalization conditions 
are evidently gauge independent. ${ }^{7}$ Fixing mixing angles by specific processes, however, has a number of potential disadvantages:

- By construction, on-shell renormalization conditions are process dependent and often destroy the symmetries between the particles that mix.

- On-shell conditions are only directly applicable to $S$-matrix elements that do not involve charged particles; otherwise the counterterms would become infrared (IR) singular. ${ }^{8}$ The problem of IR singularities can, in principle, be avoided by imposing the renormalization condition on a full physical observable, e.g. by demanding that a partial decay width does not receive any correction, but this procedure shifts processspecific real-radiation effects into the renormalization constant.

- Typically, observables and $S$-matrix elements depend not only on a mixing angle, but on other parameters as well. Upon defining the mixing-angle renormalization from such quantities, one thus absorbs corrections to the considered observable or $S$-matrix element into the mixing-angle counterterm that are related to other parameters of the model. This can be a source for unnaturally large corrections. In ref. [26] it was, e.g., demonstrated that on-shell renormalization conditions based on specific observables lead to numerically unstable results in the THDM.

The situation can be improved by considering combinations of physical observables or $S$-matrix elements that depend exclusively on a specific mixing angle and on no other parameters, so that renormalization contributions of other parameters or normalization effects systematically drop out. For the quark-mixing matrix such a renormalization scheme was proposed in ref. [16].

In order to fix the renormalization of the Higgs mixing angle $\alpha$ introduced in (2.1), we consider a set of processes involving the fields $H_{i}$ that have a simple dependence on the mixing angle $\alpha$ in LO. If the dependence on $\alpha$ in the considered observables only results from the transformation (2.1), this is typically the case.

\footnotetext{
${ }^{7}$ Note that we do not consider renormalization conditions as "on shell" that are based on mixing energies, Green functions, or formfactors as well as "matrix elements" involving unphysical degrees of freedom at some "on-shell" configurations of momenta. This includes, in particular, mixing energies of scalar bosons, of would-be Goldstone bosons with Higgs bosons or of would-be Goldstone bosons with gauge bosons. In the literature, schemes of this kind are often called "on shell" as well.

${ }^{8}$ Considering the analytic structure of one-loop 3-point functions, it can be seen that the corresponding IR singularities in decay $S$-matrix elements can only be cancelled by those of the field-renormalization constants of the external charged particles if one of the three external particles is massless and neutral, as for the photon in the electron-positron-photon vertex in QED. In other words, potential IR singularities in the $S$-matrix element used to fix a renormalization constant only cancel in this very specific case. In all other cases, IR singularities would enter the parameter renormalization constants.
} 


\subsubsection{Higgs-singlet extension of the Standard Model}

As a first specific example, we choose the decay of scalar bosons $H_{1}$ and $H_{2}$ into pairs of Z bosons in the HSESM. ${ }^{9}$ The LO vertices read

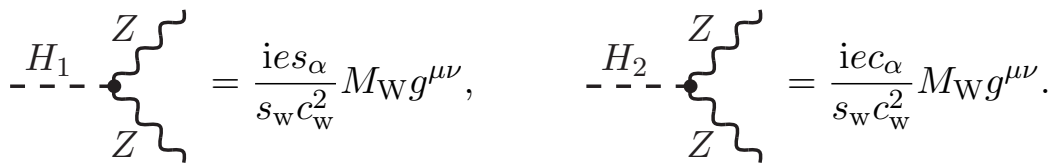

The LO matrix elements for the decays of the two Higgs bosons into a pair of $\mathrm{Z}$ bosons read

$$
\mathcal{M}_{0}^{H_{1} \rightarrow \mathrm{ZZ}}=\frac{e s_{\alpha}}{s_{\mathrm{w}} c_{\mathrm{w}}^{2}} M_{\mathrm{W}}\left(\varepsilon_{1}^{*} \cdot \varepsilon_{2}^{*}\right), \quad \mathcal{M}_{0}^{H_{2} \rightarrow \mathrm{ZZ}}=\frac{e c_{\alpha}}{s_{\mathrm{w}} c_{\mathrm{w}}^{2}} M_{\mathrm{W}}\left(\varepsilon_{1}^{*} \cdot \varepsilon_{2}^{*}\right)
$$

where $\varepsilon_{1,2}$ denote the polarization vectors of the two $\mathrm{Z}$ bosons.

At $\mathrm{LO}$, the ratio of the matrix elements $\mathcal{M}^{H_{i} \rightarrow \mathrm{ZZ}}$ for the decays of the heavy and light scalar into a pair of $\mathrm{Z}$ bosons is given by $s_{\alpha} / c_{\alpha}$. A possible renormalization condition is, thus, to require that this ratio is equal to its $\mathrm{LO}$ value, i.e.

$$
\frac{\mathcal{M}_{0}^{H_{1} \rightarrow \mathrm{ZZ}}}{\mathcal{M}_{0}^{H_{2} \rightarrow \mathrm{ZZ}}}=\frac{s_{\alpha}}{c_{\alpha}} \stackrel{!}{=} \frac{\mathcal{M}^{H_{1} \rightarrow \mathrm{ZZ}}}{\mathcal{M}^{H_{2} \rightarrow \mathrm{ZZ}}}
$$

Using the complete on-shell scheme, the renormalized NLO matrix elements can be written as

$$
\begin{aligned}
\mathcal{M}^{H_{1} \rightarrow \mathrm{ZZ}}= & \mathcal{M}_{0}^{H_{1} \rightarrow \mathrm{ZZ}}\left(1+\delta_{H_{1} Z Z}+\delta Z_{e}+\frac{1}{2} \frac{\delta M_{\mathrm{W}}^{2}}{M_{\mathrm{W}}^{2}}-\frac{\delta c_{\mathrm{w}}^{2}}{c_{\mathrm{w}}^{2}}-\frac{\delta s_{\mathrm{w}}}{s_{\mathrm{w}}}+\frac{\delta s_{\alpha}}{s_{\alpha}}\right. \\
& \left.+\delta Z_{Z Z}+\frac{1}{2} \delta Z_{11}^{H}+\frac{1}{2} \delta Z_{21}^{H} \frac{c_{\alpha}}{s_{\alpha}}\right), \\
\mathcal{M}^{H_{2} \rightarrow \mathrm{ZZ}}= & \mathcal{M}_{0}^{H_{2} \rightarrow \mathrm{ZZ}}\left(1+\delta_{H_{2} Z Z}+\delta Z_{e}+\frac{1}{2} \frac{\delta M_{\mathrm{W}}^{2}}{M_{\mathrm{W}}^{2}}-\frac{\delta c_{\mathrm{w}}^{2}}{c_{\mathrm{w}}^{2}}-\frac{\delta s_{\mathrm{w}}}{s_{\mathrm{w}}}+\frac{\delta c_{\alpha}}{c_{\alpha}}\right. \\
& \left.+\delta Z_{Z Z}+\frac{1}{2} \delta Z_{22}^{H}+\frac{1}{2} \delta Z_{12}^{H} \frac{s_{\alpha}}{c_{\alpha}}\right),
\end{aligned}
$$

where $\delta_{H_{i} Z Z}=\delta_{H_{i} Z Z}\left(M_{\mathrm{H}_{i}}^{2}\right)$ are the unrenormalized relative one-loop corrections to the respective decays, and the counterterms have been written explicitly. In particular, the two scalar fields are renormalized according to (2.4). Inserting (3.5) into (3.4) and expanding to NLO yields for the counterterm of the mixing angle $\alpha$ :

$$
\delta \alpha=c_{\alpha} s_{\alpha}\left(\delta_{H_{2} Z Z}-\delta_{H_{1} Z Z}\right)+\frac{1}{2} c_{\alpha} s_{\alpha}\left(\delta Z_{22}^{H}-\delta Z_{11}^{H}\right)+\frac{1}{2}\left(\delta Z_{12}^{H} s_{\alpha}^{2}-\delta Z_{21}^{H} c_{\alpha}^{2}\right) .
$$

Using this counterterm, the renormalized NLO matrix elements become

$$
\begin{aligned}
\mathcal{M}^{H_{i} \rightarrow \mathrm{ZZ}}= & \mathcal{M}_{0}^{H_{i} \rightarrow \mathrm{ZZ}}\left(1+\delta_{H_{1} Z Z} s_{\alpha}^{2}+\delta_{H_{2} Z Z} c_{\alpha}^{2}+\delta Z_{e}+\frac{1}{2} \frac{\delta M_{\mathrm{W}}^{2}}{M_{\mathrm{W}}^{2}}-\frac{\delta c_{\mathrm{w}}^{2}}{c_{\mathrm{w}}^{2}}-\frac{\delta s_{\mathrm{w}}}{s_{\mathrm{w}}}\right. \\
& \left.+\delta Z_{Z Z}+\frac{1}{2} \delta Z_{11}^{H} s_{\alpha}^{2}+\frac{1}{2} \delta Z_{22}^{H} c_{\alpha}^{2}+\frac{1}{2}\left(\delta Z_{21}^{H}+\delta Z_{12}^{H}\right) c_{\alpha} s_{\alpha}\right), \quad i=1,2 .
\end{aligned}
$$

\footnotetext{
${ }^{9}$ In the THDM the corresponding vertices involve $\alpha-\beta$ instead of $\alpha$ and thus can be used to renormalize this difference.
} 
Since all differences have been incorporated in the renormalization of $\alpha$, the relative corrections to the two different Higgs-boson decays become equal.

The renormalization condition (3.4) has several desirable properties:

- It is gauge independent, because it is based on physical $S$-matrix elements;

- it is symmetric with respect to the scalar fields $H_{1}$ and $H_{2}$;

- it is numerically stable for degenerate masses $M_{\mathrm{H}_{1}} \sim M_{\mathrm{H}_{2}}$;

- it has smooth limits for extreme mixing angles, i.e. for $c_{\alpha} \rightarrow 0$ or $s_{\alpha} \rightarrow 0$.

The singularities for degenerate masses $M_{\mathrm{H}_{1}} \sim M_{\mathrm{H}_{2}}$ cancel in all $S$-matrix elements for the following reason. All appearances of $\alpha$ that result from rewriting parameters of the scalar potential involve a prefactor $M_{\mathrm{H}_{1}}^{2}-M_{\mathrm{H}_{2}}^{2}$ that cancels the singularity. For all appearances of $\alpha$ introduced in the Lagrangian via the rotation (2.1) the counterterm appears always in the combinations (2.10) which after inserting the on-shell counterterm $\delta \alpha$ only depend on $\delta Z_{12}^{H}$ and $\delta Z_{21}^{H}$ via the sum

$$
\delta Z_{12}^{H}+\delta Z_{21}^{H}
$$

Upon using (2.5), this becomes

$$
\delta Z_{12}^{H}+\delta Z_{21}^{H}=2 \frac{\Sigma_{12}^{H}\left(M_{\mathrm{H}_{2}}^{2}\right)-\Sigma_{12}^{H}\left(M_{\mathrm{H}_{1}}^{2}\right)}{M_{\mathrm{H}_{1}}^{2}-M_{\mathrm{H}_{2}}^{2}},
$$

which is finite for degenerate masses, $M_{\mathrm{H}_{1}} \rightarrow M_{\mathrm{H}_{2}}$, and moreover all momentum-independent contributions to the mixing energy, such as tadpole contributions, cancel therein. These statements hold for all other on-shell schemes for $\alpha$ discussed in this section.

Still, the condition (3.4) has some disadvantages:

- It can be directly applied to decay processes involving only electrically neutral external particles. For charged external particles, the renormalization constant $\delta \alpha$ becomes IR singular.

- Depending on the masses of the external particles, the form factors have to be evaluated at phase-space points in the unphysical region. ${ }^{10}$ This is, for instance, the case in (3.6) if $\mathrm{H}_{1}$ or $\mathrm{H}_{2}$ is identified with the observed Higgs state of mass $125 \mathrm{GeV}$.

All these drawbacks can be lifted upon introducing extra neutral fields with a simple coupling structure that allows us to fix the renormalization of the mixing angles while recovering the original theory upon sending the extra couplings to zero.

As an example for this procedure, we consider the HSESM and add an additional fermion singlet field $\psi$ with the Lagrangian

$$
\mathcal{L}_{\psi}=\mathrm{i} \bar{\psi} \not \partial \psi-y_{\psi} \sigma \bar{\psi} \psi
$$

\footnotetext{
${ }^{10}$ At least for decays at the one-loop level this does not constitute an obstacle. In this case the relevant 3-point functions can be analytically continued to the unphysical region as discussed in ref. [44].
} 
In terms of scalar fields in the basis of mass eigenstates, this becomes

$$
\mathcal{L}_{\psi}=\mathrm{i} \bar{\psi} \not \partial \psi-y_{\psi}\left(v_{1}+H_{1} c_{\alpha}-H_{2} s_{\alpha}\right) \bar{\psi} \psi
$$

Considering the limit of a vanishing Yukawa coupling, $y_{\psi} \rightarrow 0$, we recover the original HSESM with an additional massless fermion $\psi\left(m_{\psi}=y_{\psi} v_{1} \rightarrow 0\right)$, which completely decouples from all other particles, i.e. we effectively recover the original theory. We require that the ratio of matrix elements for the decays of the two scalar Higgs bosons into a $\psi \bar{\psi}$ pair of singlets is equal to its leading-order value $-c_{\alpha} / s_{\alpha}$ in the limit of vanishing coupling $y_{\psi}$ :

$$
\frac{\mathcal{M}^{H_{1} \rightarrow \psi \psi}}{\mathcal{M}^{H_{2} \rightarrow \psi \psi}} \stackrel{!}{=} \frac{\mathcal{M}_{0}^{H_{1} \rightarrow \psi \psi}}{\mathcal{M}_{0}^{H_{2} \rightarrow \psi \psi}} \propto-\frac{c_{\alpha}}{s_{\alpha}},
$$

where the proportionality factor, which is not spelled out, only contains the ratio of spinor chains (with different kinematics), but no other model parameters. Since the ratio is based on matrix elements for the decay of massive scalars into massless neutral fermions these are in the physical region, and no IR singularities occur. Moreover, since all NLO vertex corrections in $\mathcal{M}^{H_{i} \rightarrow \psi \psi}$ tend to zero at least quadratically in $y_{\psi}$ and thus drop out in the limit $y_{\psi} \rightarrow 0,{ }^{11}$ we obtain for the counterterm

$$
\delta \alpha=\frac{1}{2}\left(\delta Z_{11}^{H}-\delta Z_{22}^{H}\right) c_{\alpha} s_{\alpha}+\frac{1}{2}\left(\delta Z_{12}^{H} c_{\alpha}^{2}-\delta Z_{21}^{H} s_{\alpha}^{2}\right) .
$$

Thus, owing to the simple structure of the model, all vertex corrections drop out, and the mixing-angle counterterm is fixed by a gauge-independent combination of field-renormalization constants only. Note also that the spinor chains suppressed in (3.12) do not enter the final result for $\delta \alpha$, since they cancel in the ratios $\mathcal{M}^{H_{i} \rightarrow \psi \psi} / \mathcal{M}_{0}^{H_{i} \rightarrow \psi \psi}$.

\subsubsection{Two-Higgs-doublet model}

In order to formulate on-shell renormalization conditions for the THDM, we add two righthanded fermion singlets to the Lagrangian: $\nu_{1 \mathrm{R}}$ transforming under the extra $\mathbb{Z}_{2}$ symmetry as $\nu_{1 \mathrm{R}} \rightarrow-\nu_{1 \mathrm{R}}$ and $\nu_{2 \mathrm{R}}$ transforming as $\nu_{2 \mathrm{R}} \rightarrow \nu_{2 \mathrm{R}}$, so that $\nu_{i \mathrm{R}}$ can only receive a Yukawa coupling to $\Phi_{i}$. The additional Lagrangian reads

$$
\mathcal{L}_{\nu_{\mathrm{R}}}=\mathrm{i} \bar{\nu}_{1 \mathrm{R}} \not \partial \nu_{1 \mathrm{R}}+\mathrm{i} \bar{\nu}_{2 \mathrm{R}} \not \partial \nu_{2 \mathrm{R}}-\left[y_{\nu_{1}} \bar{L}_{1 \mathrm{~L}}\left(\mathrm{i} \sigma_{2} \Phi_{1}^{*}\right) \nu_{1 \mathrm{R}}+y_{\nu_{2}} \bar{L}_{2 \mathrm{~L}}\left(\mathrm{i} \sigma_{2} \Phi_{2}^{*}\right) \nu_{2 \mathrm{R}}+\text { h.c. }\right],
$$

where $y_{\nu_{i}}$ are new Yukawa couplings that are considered in the limit $y_{\nu_{i}} \rightarrow 0$. The fields $L_{i \mathrm{~L}}=\left(\nu_{i}, l_{i}\right)_{\mathrm{L}}^{\mathrm{T}}$ are left-handed lepton doublets of the SM, say the electron-neutrino and muon-neutrino doublets, $\Phi_{i}$ are the two Higgs-doublet fields, and $\sigma_{2}$ the second Pauli matrix. Upon inserting the representations of the doublet fields this leads to

$$
\begin{aligned}
\mathcal{L}_{\nu_{\mathrm{R}}}= & -\frac{1}{\sqrt{2}}\left[y_{\nu_{1}} \bar{\nu}_{1 \mathrm{~L}} \nu_{1 \mathrm{R}}\left(v c_{\beta}+H_{1} c_{\alpha}-H_{2} s_{\alpha}+\mathrm{i} A_{0} s_{\beta}-\mathrm{i} G_{0} c_{\beta}\right)+\text { h.c. }\right] \\
& -\frac{1}{\sqrt{2}}\left[y_{\nu_{2}} \bar{\nu}_{2 \mathrm{~L}} \nu_{2 \mathrm{R}}\left(v s_{\beta}+H_{1} s_{\alpha}+H_{2} c_{\alpha}-\mathrm{i} A_{0} c_{\beta}-\mathrm{i} G_{0} s_{\beta}\right)+\text { h.c. }\right] \\
& +\ldots,
\end{aligned}
$$

\footnotetext{
${ }^{11}$ No CP-odd effective coupling $\propto \bar{\psi} \gamma_{5} \psi$ is induced by loops.
} 
where we suppressed terms involving charged scalar Higgs and would-be Goldstone-boson fields. For non-zero couplings $y_{\nu_{i}}$, the neutrinos $\nu_{1}$ and $\nu_{2}$ correspond to massive Dirac fermions without generation mixing owing to the conserved lepton number. In the limit $y_{\nu_{i}} \rightarrow 0$, the neutrinos become massless, and the right-handed parts decouple.

Renormalization of $\boldsymbol{\alpha}$. The renormalization of the angle $\alpha$ can be fixed upon requiring that the ratio of the matrix elements for the decays $H_{i} \rightarrow \nu_{1} \bar{\nu}_{1}$ is the same at LO and NLO,

$$
\frac{\mathcal{M}^{H_{1} \rightarrow \nu_{1} \bar{\nu}_{1}}}{\mathcal{M}^{H_{2} \rightarrow \nu_{1} \bar{\nu}_{1}}} \stackrel{!}{=} \frac{\mathcal{M}_{0}^{H_{1} \rightarrow \nu_{1} \bar{\nu}_{1}}}{\mathcal{M}_{0}^{H_{2} \rightarrow \nu_{1} \bar{\nu}_{1}}} .
$$

This leads to the renormalization constant

$$
\delta \alpha=\left(\delta_{H_{1} \nu_{1} \bar{\nu}_{1}}-\delta_{H_{2} \nu_{1} \bar{\nu}_{1}}\right) c_{\alpha} s_{\alpha}+\frac{1}{2}\left(\delta Z_{11}^{H}-\delta Z_{22}^{H}\right) c_{\alpha} s_{\alpha}+\frac{1}{2}\left(\delta Z_{12}^{H} c_{\alpha}^{2}-\delta Z_{21}^{H} s_{\alpha}^{2}\right),
$$

where $\delta_{H_{i} \nu_{1} \bar{\nu}_{1}}$ represent the unrenormalized relative one-loop corrections to the decays $H_{i} \rightarrow \nu_{1} \bar{\nu}_{1}$. Alternatively, if the ratio of the matrix elements for the decays $H_{i} \rightarrow \nu_{2} \bar{\nu}_{2}$ is used to fix the renormalization of $\alpha$, this leads to the renormalization constant

$$
\delta \alpha=\left(\delta_{H_{2} \nu_{2} \bar{\nu}_{2}}-\delta_{H_{1} \nu_{2} \bar{\nu}_{2}}\right) c_{\alpha} s_{\alpha}+\frac{1}{2}\left(\delta Z_{22}^{H}-\delta Z_{11}^{H}\right) c_{\alpha} s_{\alpha}+\frac{1}{2}\left(\delta Z_{12}^{H} s_{\alpha}^{2}-\delta Z_{21}^{H} c_{\alpha}^{2}\right) .
$$

The explicit form of the vertex correction factors $\delta_{H_{1} \nu_{1} \bar{\nu}_{1}}$, etc., is given in appendix D. At $\mathrm{NLO}$, these loop corrections respect the chiral structures of the respective underlying LO couplings; beyond NLO this might not be the case anymore, so that one would have to write the renormalization conditions in terms of the form factors that correspond to the LO couplings.

Renormalization of $\boldsymbol{\beta}$. The renormalization of the angle $\beta$ can be fixed by demanding that the ratio of the matrix elements for the decays $H_{1} \rightarrow \nu_{i} \bar{\nu}_{i}$ and $A_{0} \rightarrow \nu_{i} \bar{\nu}_{i}$ is the same at LO and NLO for one of the two neutrinos $\nu_{i}$. Specifying $\nu_{i}$ to $\nu_{1}$, means

$$
\frac{\mathcal{M}^{A_{0} \rightarrow \nu_{1} \bar{\nu}_{1}}}{\mathcal{M}^{H_{1} \rightarrow \nu_{1} \bar{\nu}_{1}}} \stackrel{!}{=} \frac{\mathcal{M}_{0}^{A_{0} \rightarrow \nu_{1} \bar{\nu}_{1}}}{\mathcal{M}_{0}^{H_{1} \rightarrow \nu_{1} \bar{\nu}_{1}}} \propto \frac{s_{\beta}}{c_{\alpha}},
$$

where again the suppressed proportionality factor is given by a ratio of spinor chains, but does not contain further model parameters. This results in

$$
\delta \beta=\frac{s_{\beta}}{c_{\beta}}\left(\delta_{H_{1} \nu_{1} \bar{\nu}_{1}}-\delta_{A_{0} \nu_{1} \bar{\nu}_{1}}-\frac{1}{2}\left(\delta Z_{A_{0} A_{0}}-\delta Z_{11}^{H}\right)-\frac{1}{2} \frac{s_{\alpha}}{c_{\alpha}} \delta Z_{21}^{H}\right)+\frac{1}{2} \delta Z_{G_{0} A_{0}}-\frac{s_{\beta}}{c_{\beta}} \frac{s_{\alpha}}{c_{\alpha}} \delta \alpha,
$$

where $\delta_{A_{0} \nu_{1} \bar{\nu}_{1}}$ denotes the unrenormalized relative one-loop corrections to the matrix element for the decay $A_{0} \rightarrow \nu_{1} \bar{\nu}_{1}$. Upon inserting the renormalization constant $\delta \alpha$ from (3.17) this becomes

$$
\begin{aligned}
\delta \beta= & \frac{s_{\beta}}{c_{\beta}}\left(c_{\alpha}^{2} \delta_{H_{1} \nu_{1} \bar{\nu}_{1}}+s_{\alpha}^{2} \delta_{H_{2} \nu_{1} \bar{\nu}_{1}}-\delta_{A_{0} \nu_{1} \bar{\nu}_{1}}\right. \\
& \left.-\frac{1}{2}\left[\delta Z_{A_{0} A_{0}}-c_{\alpha}^{2} \delta Z_{11}^{H}-s_{\alpha}^{2} \delta Z_{22}^{H}+c_{\alpha} s_{\alpha}\left(\delta Z_{12}^{H}+\delta Z_{21}^{H}\right)\right]\right)+\frac{1}{2} \delta Z_{G_{0} A_{0}},
\end{aligned}
$$


which involves only vertex corrections for neutrino $\nu_{1}$. Note that we would get the same relation if we had fixed $\delta \beta$ from the ratio of $A_{0} \rightarrow \nu_{1} \bar{\nu}_{1}$ and $H_{2} \rightarrow \nu_{1} \bar{\nu}_{1}$ matrix elements by virtue of (3.16).

Alternatively, $\delta \alpha$ and $\delta \beta$ can be fixed from analogous matrix elements involving only neutrino $\nu_{2}$. In this case, for $\delta \beta$ we demand

$$
\frac{\mathcal{M}^{A_{0} \rightarrow \nu_{2} \bar{\nu}_{2}}}{\mathcal{M}^{H_{1} \rightarrow \nu_{2} \bar{\nu}_{2}}} \stackrel{!}{=} \frac{\mathcal{M}_{0}^{A_{0} \rightarrow \nu_{2} \bar{\nu}_{2}}}{\mathcal{M}_{0}^{H_{1} \rightarrow \nu_{2} \bar{\nu}_{2}}} \propto-\frac{c_{\beta}}{s_{\alpha}},
$$

resulting in

$$
\begin{aligned}
\delta \beta= & \frac{c_{\beta}}{s_{\beta}}\left(\delta_{A_{0} \nu_{2} \bar{\nu}_{2}}-\delta_{H_{1} \nu_{2} \bar{\nu}_{2}}+\frac{1}{2}\left(\delta Z_{A_{0} A_{0}}-\delta Z_{11}^{H}\right)-\frac{1}{2} \frac{c_{\alpha}}{s_{\alpha}} \delta Z_{21}^{H}\right) \\
& +\frac{1}{2} \delta Z_{G_{0} A_{0}}-\frac{c_{\beta}}{s_{\beta}} \frac{c_{\alpha}}{s_{\alpha}} \delta \alpha,
\end{aligned}
$$

which can be further processed with $\delta \alpha$ from (3.18) to yield

$$
\begin{aligned}
\delta \beta= & \frac{c_{\beta}}{s_{\beta}}\left(\delta_{A_{0} \nu_{2} \bar{\nu}_{2}}-s_{\alpha}^{2} \delta_{H_{1} \nu_{2} \bar{\nu}_{2}}-c_{\alpha}^{2} \delta_{H_{2} \nu_{2} \bar{\nu}_{2}}\right. \\
& \left.+\frac{1}{2}\left[\delta Z_{A_{0} A_{0}}-s_{\alpha}^{2} \delta Z_{11}^{H}-c_{\alpha}^{2} \delta Z_{22}^{H}-c_{\alpha} s_{\alpha}\left(\delta Z_{12}^{H}+\delta Z_{21}^{H}\right)\right]\right)+\frac{1}{2} \delta Z_{G_{0} A_{0}} .
\end{aligned}
$$

The conditions (3.21) and (3.24) become singular for $c_{\beta} \rightarrow 0$ or $s_{\beta} \rightarrow 0$, respectively. Since in the phenomenological applications of the THDM, $c_{\beta}$ and $s_{\beta}$ are always non-vanishing, this does not lead to a singularity but can cause artificial enhancements.

The renormalization scheme based on the conditions (3.17) and (3.21) involving only $\nu_{1}$ is called OS1 in the following, the one based on (3.18) and (3.24) involving only $\nu_{2}$ is called OS2.

The conditions (3.19) and (3.22) do not directly apply to $\beta$, but to a combination of $\alpha$ and $\beta$. A condition that fixes $\beta$ directly can be obtained by using the decays into both neutrino singlets and requiring

$$
\frac{\mathcal{M}^{H_{1} \rightarrow \nu_{1} \bar{\nu}_{1}}}{\mathcal{M}^{H_{1} \rightarrow \nu_{2} \bar{\nu}_{2}}} \frac{\mathcal{M}^{H_{2} \rightarrow \nu_{1} \bar{\nu}_{1}}}{\mathcal{M}^{H_{2} \rightarrow \nu_{2} \bar{\nu}_{2}}}\left(\frac{\mathcal{M}^{A_{0} \rightarrow \nu_{2} \bar{\nu}_{2}}}{\mathcal{M}^{A_{0} \rightarrow \nu_{1} \bar{\nu}_{1}}}\right)^{2} \stackrel{!}{=} \frac{\mathcal{M}_{0}^{H_{1} \rightarrow \nu_{1} \bar{\nu}_{1}}}{\mathcal{M}_{0}^{H_{1} \rightarrow \nu_{2} \bar{\nu}_{2}}} \frac{\mathcal{M}_{0}^{H_{2} \rightarrow \nu_{1} \bar{\nu}_{1}}}{\mathcal{M}_{0}^{H_{2} \rightarrow \nu_{2} \bar{\nu}_{2}}}\left(\frac{\mathcal{M}_{0}^{A_{0} \rightarrow \nu_{2} \bar{\nu}_{2}}}{\mathcal{M}_{0}^{A_{0} \rightarrow \nu_{1} \bar{\nu}_{1}}}\right)^{2}=-\frac{c_{\beta}^{2}}{s_{\beta}^{2}} .
$$

Note that the spinor chains all cancel within the multiple ratio. This condition leads to the counterterm

$$
\begin{aligned}
\delta \beta= & \frac{1}{2} c_{\beta} s_{\beta}\left(\delta_{H_{1} \nu_{1} \bar{\nu}_{1}}+\delta_{H_{2} \nu_{1} \bar{\nu}_{1}}-2 \delta_{A_{0} \nu_{1} \bar{\nu}_{1}}-\delta_{H_{1} \nu_{2} \bar{\nu}_{2}}-\delta_{H_{2} \nu_{2} \bar{\nu}_{2}}+2 \delta_{A_{0} \nu_{2} \bar{\nu}_{2}}\right) \\
& -\frac{c_{\beta} s_{\beta}}{4 c_{\alpha} s_{\alpha}}\left(\delta Z_{12}^{H}+\delta Z_{21}^{H}\right)+\frac{1}{2} \delta Z_{G_{0} A_{0}},
\end{aligned}
$$

which is regular for $s_{\beta} \rightarrow 0$ of $c_{\beta} \rightarrow 0$, but potentially singular for $s_{\alpha} \rightarrow 0$ or $c_{\alpha} \rightarrow 0$.

A condition leading to a counterterm that is regular in all these limits can be constructed upon using linear combinations of observable quantities from different processes. 
To this end, we consider on-shell form factors instead of complete matrix elements. For the scalar decays into (nearly) massless fermions we write the matrix elements as

$$
\mathcal{M}^{H_{i} \rightarrow \nu_{j} \bar{\nu}_{j}}=\left[\bar{u}_{\nu} v_{\nu}\right]_{H_{i}} F^{H_{i} \rightarrow \nu_{j} \bar{\nu}_{j}}, \quad \mathcal{M}^{A_{0} \rightarrow \nu_{j} \bar{\nu}_{j}}=\left[\bar{u}_{\nu} \mathrm{i} \gamma_{5} v_{\nu}\right]_{A_{0}} F^{A_{0} \rightarrow \nu_{j} \bar{\nu}_{j}}
$$

where $\bar{u}_{\nu}$ and $v_{\nu}$ are the spinors of the final-state fermions and antifermions, and $[\ldots]_{H_{i} / A_{0}}$ indicates the decay kinematics of the spinor chain. The functions $F^{H_{i} \rightarrow \nu_{j} \bar{\nu}_{j}}$ and $F^{A_{0} \rightarrow \nu_{j} \bar{\nu}_{j}}$ denote the formfactors for the decays into neutrinos $\nu_{j}$ of type $j=1,2$. The LO formfactors follow directly from the Lagrangian (3.15):

$$
\begin{array}{lll}
F_{0}^{H_{1} \rightarrow \nu_{1} \bar{\nu}_{1}}=-\frac{1}{\sqrt{2}} y_{\nu_{1}} c_{\alpha}, & F_{0}^{H_{2} \rightarrow \nu_{1} \bar{\nu}_{1}}=\frac{1}{\sqrt{2}} y_{\nu_{1}} s_{\alpha}, & F_{0}^{A_{0} \rightarrow \nu_{1} \bar{\nu}_{1}}=-\frac{1}{\sqrt{2}} y_{\nu_{1}} s_{\beta}, \\
F_{0}^{H_{1} \rightarrow \nu_{2} \bar{\nu}_{2}}=-\frac{1}{\sqrt{2}} y_{\nu_{2}} s_{\alpha}, & F_{0}^{H_{2} \rightarrow \nu_{2} \bar{\nu}_{2}}=-\frac{1}{\sqrt{2}} y_{\nu_{2}} c_{\alpha}, & F_{0}^{A_{0} \rightarrow \nu_{2} \bar{\nu}_{2}}=\frac{1}{\sqrt{2}} y_{\nu_{2}} c_{\beta} .
\end{array}
$$

As renormalization condition we require that the following LO relation holds also at higher orders:

$$
\begin{aligned}
& 0=\frac{F_{0}^{A_{0} \rightarrow \nu_{1} \bar{\nu}_{1}}}{c_{\alpha} F_{0}^{H_{1} \rightarrow \nu_{1} \bar{\nu}_{1}}-s_{\alpha} F_{0}^{H_{2} \rightarrow \nu_{1} \bar{\nu}_{1}}} c_{\beta}+\frac{F_{0}^{A_{0} \rightarrow \nu_{2} \bar{\nu}_{2}}}{s_{\alpha} F_{0}^{H_{1} \rightarrow \nu_{2} \bar{\nu}_{2}}+c_{\alpha} F_{0}^{H_{2} \rightarrow \nu_{2} \bar{\nu}_{2}}} s_{\beta} \\
& \stackrel{!}{=} \frac{F^{A_{0} \rightarrow \nu_{1} \bar{\nu}_{1}}}{c_{\alpha} F^{H_{1} \rightarrow \nu_{1} \bar{\nu}_{1}}-s_{\alpha} F^{H_{2} \rightarrow \nu_{1} \bar{\nu}_{1}}} c_{\beta}+\frac{F^{A_{0} \rightarrow \nu_{2} \bar{\nu}_{2}}}{s_{\alpha} F^{H_{1} \rightarrow \nu_{2} \bar{\nu}_{2}}+c_{\alpha} F^{H_{2} \rightarrow \nu_{2} \bar{\nu}_{2}}} s_{\beta} .
\end{aligned}
$$

This fixes the counterterm for the mixing angle $\beta$ to

$$
\begin{aligned}
\delta \beta= & \frac{1}{2} c_{\beta} s_{\beta}\left[\left(c_{\alpha}^{2}-s_{\alpha}^{2}\right)\left(\delta Z_{11}^{H}-\delta Z_{22}^{H}\right)-2 c_{\alpha} s_{\alpha}\left(\delta Z_{12}^{H}+\delta Z_{21}^{H}\right)\right]+\frac{1}{2} \delta Z_{G_{0} A_{0}} \\
& +c_{\beta} s_{\beta}\left(\delta_{A_{0} \nu_{2} \bar{\nu}_{2}}+c_{\alpha}^{2} \delta_{H_{1} \nu_{1} \bar{\nu}_{1}}+s_{\alpha}^{2} \delta_{H_{2} \nu_{1} \bar{\nu}_{1}}-\delta_{A_{0} \nu_{1} \bar{\nu}_{1}}-s_{\alpha}^{2} \delta_{H_{1} \nu_{2} \bar{\nu}_{2}}-c_{\alpha}^{2} \delta_{H_{2} \nu_{2} \bar{\nu}_{2}}\right)
\end{aligned}
$$

This result is non-singular in all limits $s_{\alpha} \rightarrow 0, c_{\alpha} \rightarrow 0, s_{\beta} \rightarrow 0$, or $c_{\beta} \rightarrow 0$.

The above on-shell renormalization conditions for mixing angles in the HSESM and THDM depend on the introduction of specific auxiliary fields. While this method can in principle be generalized to more general theories, we are not able to provide a simple specific recipe for the on-shell renormalization of mixing angles or mixing matrices in general. As far as we can see, this has to be investigated anew for each theory, but the shown examples can certainly serve as guidelines.

\subsection{Renormalization of mixing angles based on symmetries}

\subsubsection{Rigid symmetry and wave-function renormalization for physical states}

The renormalization of mixing matrices can be related to the wave-function renormalization of external fields upon using rigid symmetry, i.e. the symmetry under global gauge transformations, of the Lagrangian. Again, we specifically consider the THDM and the HSESM, where the CP-even scalar fields in the symmetric and mass-eigenstate bases are related via (2.1).

A theory with a spontaneously broken gauge symmetry can be renormalized using the renormalization constants for fields and dimensionless parameters from the symmetric 
phase [45-51]. In particular, the counterterms for the dimensionless parameters and fields can be directly taken from the symmetric formulation. Once the counterterms for the mass parameters are adjusted appropriately, all UV divergences cancel while the external lines in $S$-matrix elements still require additional finite wave-function renormalization. For the SM such a renormalization scheme was used in ref. [52]. In the case considered here, the relevant renormalization transformations read

$$
\begin{aligned}
\alpha_{\mathrm{B}} & =\alpha+\delta \alpha \\
\boldsymbol{\eta}_{\mathrm{B}} & =\left(Z^{\eta}\right)^{1 / 2} \boldsymbol{\eta}, \\
\left(Z^{\eta}\right)^{1 / 2} & =\left(\begin{array}{cc}
\left(Z_{1}^{\eta}\right)^{1 / 2} & 0 \\
0 & \left(Z_{2}^{\eta}\right)^{1 / 2}
\end{array}\right)=1+\frac{1}{2} \delta Z^{\eta}=\left(\begin{array}{cc}
1+\frac{1}{2} \delta Z_{1}^{\eta} & 0 \\
0 & 1+\frac{1}{2} \delta Z_{2}^{\eta}
\end{array}\right),
\end{aligned}
$$

where the components $\eta_{1}$ and $\eta_{2}$ of $\boldsymbol{\eta}$ belong to different multiplets of the gauge group.

Consistency of eqs. (2.1) and (3.33) requires that at least the divergent parts of the renormalization constants in both schemes are related via

$$
\left.\left(Z^{H}\right)^{1 / 2}\right|_{\mathrm{UV}}=\left.R^{\mathrm{T}}(\alpha+\delta \alpha)\left(Z^{\eta}\right)^{1 / 2} R(\alpha)\right|_{\mathrm{UV}}=\left.\left[R^{\mathrm{T}}(\alpha)+\delta R^{\mathrm{T}}(\alpha, \delta \alpha)\right]\left(Z^{\eta}\right)^{1 / 2} R(\alpha)\right|_{\mathrm{UV}}
$$

Using $0=\delta\left(R^{\mathrm{T}} R\right)=\delta R^{\mathrm{T}} R+R^{\mathrm{T}} \delta R$, we get

$$
\left.\delta Z^{H}\right|_{\mathrm{UV}}=-\left.2 R^{\mathrm{T}}(\alpha) \delta R(\alpha, \delta \alpha)\right|_{\mathrm{UV}}+\left.R^{\mathrm{T}}(\alpha) \delta Z^{\eta} R(\alpha)\right|_{\mathrm{UV}}
$$

where

$$
\delta R(\alpha, \delta \alpha)=\left(\begin{array}{cc}
-s_{\alpha} & -c_{\alpha} \\
c_{\alpha} & -s_{\alpha}
\end{array}\right) \delta \alpha, \quad R^{\mathrm{T}}(\alpha) \delta R(\alpha, \delta \alpha)=\left(\begin{array}{cc}
0 & -\delta \alpha \\
\delta \alpha & 0
\end{array}\right) .
$$

This implies

$$
\begin{aligned}
\left.\delta Z_{11}^{H}\right|_{\mathrm{UV}} & =\left.c_{\alpha}^{2} \delta Z_{1}^{\eta}\right|_{\mathrm{UV}}+\left.s_{\alpha}^{2} \delta Z_{2}^{\eta}\right|_{\mathrm{UV}} \\
\left.\delta Z_{22}^{H}\right|_{\mathrm{UV}} & =\left.s_{\alpha}^{2} \delta Z_{1}^{\eta}\right|_{\mathrm{UV}}+\left.c_{\alpha}^{2} \delta Z_{2}^{\eta}\right|_{\mathrm{UV}} \\
\left.\delta Z_{12}^{H}\right|_{\mathrm{UV}}+\left.\delta Z_{21}^{H}\right|_{\mathrm{UV}} & =\left.2 c_{\alpha} s_{\alpha}\left(\delta Z_{2}^{\eta}-\delta Z_{1}^{\eta}\right)\right|_{\mathrm{UV}} \\
\left.\delta Z_{12}^{H}\right|_{\mathrm{UV}}-\left.\delta Z_{21}^{H}\right|_{\mathrm{UV}} & =\left.4 \delta \alpha\right|_{\mathrm{UV}}
\end{aligned}
$$

Thus, we find, in particular, a relation between the renormalization constant of the mixing angle $\delta \alpha$ and the non-diagonal field renormalization constants of the scalar Higgs-field pair. While the relations (3.37)-(3.40) hold for the UV-divergent parts, as for instance discussed in ref. [29], not all of them can be required simultaneously for the finite parts if the field renormalization is fixed in the complete on-shell scheme.

On the other hand, (3.40) can be used to fix the renormalization of the mixing angle $\alpha$ in terms of on-shell field renormalization constants of the scalar Higgs fields, i.e. we can define

$$
\delta \alpha=\frac{1}{4}\left(\delta Z_{12}^{H}-\delta Z_{21}^{H}\right)=\frac{\Sigma_{12}^{H}\left(M_{\mathrm{H}_{2}}^{2}\right)+\Sigma_{12}^{H}\left(M_{\mathrm{H}_{1}}^{2}\right)}{2\left(M_{\mathrm{H}_{1}}^{2}-M_{\mathrm{H}_{2}}^{2}\right)},
$$


where we expressed the non-diagonal field renormalization constants by the non-diagonal mixing energy upon using the on-shell renormalization conditions (2.5). This renormalization condition has been introduced by Kanemura et al. in ref. [23] and used in ref. [26] both in the tadpole scheme of ref. [23] and the FJTS.

As discussed in section 2, the counterterm to the mixing angle appears, besides in the regular expression $\left(M_{\mathrm{H}_{1}}^{2}-M_{\mathrm{H}_{2}}^{2}\right) \delta \alpha$, only in the combinations (2.10) in $S$-matrix elements. Thus, when using the renormalization condition (3.41), only the combination

$$
\frac{1}{2} \delta Z_{12}^{H}-\delta \alpha=\delta \alpha+\frac{1}{2} \delta Z_{21}^{H}=\frac{1}{4}\left(\delta Z_{12}^{H}+\delta Z_{21}^{H}\right)
$$

remains. According to (3.9), this is finite for degenerate masses, $M_{\mathrm{H}_{1}} \rightarrow M_{\mathrm{H}_{2}}$, and moreover all momentum-independent contributions to the mixing energy, such as tadpole contributions, cancel therein.

Renormalizing mixing angles through appropriately chosen field renormalization constants has several advantages:

- The symmetry between the different states is respected;

- if the limit of vanishing mixing is protected by a symmetry implying $\Sigma_{12}^{H} \rightarrow 0$ for $\alpha \rightarrow 0$, this is not violated by the renormalization condition, i.e. $\delta \alpha \rightarrow 0$ for $\alpha \rightarrow 0$;

- there is a smooth limit for degenerate masses, i.e. the renormalization is numerically stable and does not lead to enhanced corrections;

- there is no problem with IR singularities since the mixing energies are free of such contributions.

An apparent drawback is the gauge dependence of the field renormalization constants. However, we can choose a specific gauge to calculate the counterterms for the mixing angles and fix these counterterms in this gauge. ${ }^{12}$ Then, we can vary the gauge as usual (but keeping $\delta \alpha$ fixed in the original gauge), and $S$-matrix elements are gauge independent for fixed $\delta \alpha$. It remains to pick a suitable gauge to fix the mixing-angle counterterm. In order not to introduce artificially large parameters, the 't Hooft-Feynman gauge $(\xi=1)$ can be chosen. This can be done in the conventional formalism or, preferably, in the background-field method (BFM) where rigid symmetry holds for the effective action by construction (see section 3.3.3). Once the mixing-angle renormalization is fixed in this way, relations between observables can be calculated as usual. The gauge independence of $S$-matrix elements ensures that no singularities appear if the calculation is done in a different gauge. This procedure relies on the fact that unrenormalized $S$-matrix elements with appropriate LSZ factors are gauge independent as functions of the bare parameters. This requires that all relations between bare parameters are gauge independent which is the case in the FJTS scheme, but not in the tadpole schemes of refs. [9, 39].

\footnotetext{
${ }^{12}$ The suggestions of refs. [12, 17] and the proposal of ref. [30] for the renormalization of $\beta$ follow a similar reasoning.
} 
For the sake of clarity, let us assume that the mixing angle counterterm $\delta \alpha\left(\xi_{0}\right)$ has been fixed from $\delta Z_{i j}^{H}\left(\xi_{0}\right)$ in a specific gauge, with fixed gauge parameter $\xi_{0}$. If the $S$-matrix elements are calculated in a different gauge (with gauge parameter $\xi \neq \xi_{0}$ ), but with the fixed counterterm $\delta \alpha\left(\xi_{0}\right)$, the cancellations of potential singularities for degenerate masses occurring in (3.42) and (3.9) are not obvious anymore. The contributions of such terms can be studied by considering the gauge dependence of (3.41). The gauge-dependent parts of the one-loop mixing energy obey the Nielsen identity [53]

$$
\partial_{\xi} \Sigma_{i j}(s)=\Lambda_{i j}(s)\left(s-M_{\mathrm{H}_{j}}^{2}\right)+\left(s-M_{\mathrm{H}_{i}}^{2}\right) \Lambda_{i j}^{\prime}(s),
$$

where $\Lambda_{i j}^{(\prime)}$ are one-loop Green functions involving the operators for the BRST transformations of the fields $H_{i}$. This implies,

$$
\partial_{\xi} \frac{\Sigma_{i j}\left(M_{\mathrm{H}_{i}}^{2}\right)+\Sigma_{i j}\left(M_{\mathrm{H}_{j}}^{2}\right)}{M_{\mathrm{H}_{i}}^{2}-M_{\mathrm{H}_{j}}^{2}}=\Lambda_{i j}\left(M_{\mathrm{H}_{i}}^{2}\right)-\Lambda_{i j}^{\prime}\left(M_{\mathrm{H}_{j}}^{2}\right),
$$

i.e. the gauge-dependent part is regular for $M_{\mathrm{H}_{i}} \rightarrow M_{\mathrm{H}_{j}}$ and free of momentum-independent contributions.

Using (2.5) and (3.35) for higher-dimensional matrices, the renormalization condition (3.41) can be straightforwardly generalized to models with more physical scalar fields as

$$
R^{\mathrm{T}}(\boldsymbol{\alpha}) \delta R(\boldsymbol{\alpha}, \delta \boldsymbol{\alpha})=\frac{1}{4}\left[\left(\delta Z^{H}\right)^{\mathrm{T}}-\delta Z^{H}\right]
$$

which fixes the finite parts in $\delta \boldsymbol{\alpha}$ by definition. Moreover, (3.35) implies

$$
\left.\frac{1}{2}\left[\left(\delta Z^{H}\right)^{\mathrm{T}}+\delta Z^{H}\right]\right|_{\mathrm{UV}}=\left.R^{\mathrm{T}}(\boldsymbol{\alpha}) \delta Z^{\eta} R(\boldsymbol{\alpha})\right|_{\mathrm{UV}} .
$$

In the light of the discussion of this section, the original proposal for the renormalization of the quark-mixing matrix [8] in the SM turns out to be viable. Strictly speaking, this requires the use of the gauge-independent FJTS scheme. However, since there are no tadpole contributions to the quark mixing energies and thus to the non-diagonal fermion field renormalization constants in the SM, the renormalization condition of ref. [8] for the quarkmixing matrix is not affected by the tadpole scheme. Thus, after fixing the counterterms for the quark-mixing matrix in the 't Hooft-Feynman gauge, (gauge-independent) observables can be calculated as usual. Moreover, for the relevant one-loop fermion self-energies the results of the BFM are equivalent to those of the conventional formalism.

\subsubsection{Rigid symmetry and wave-function renormalization for unphysical states}

The renormalization condition (3.41) can also be used for the mixing with the wouldbe Goldstone bosons as for instance in the pseudoscalar and charged-scalar sector of the THDM. Using the fact that would-be Goldstone bosons are massless upon disregarding the gauge-fixing term that is not renormalized in linear gauges, we obtain for instance for mixing of the pseudoscalar with the would-be Goldstone boson,

$$
\begin{aligned}
\delta Z_{G_{0} A_{0}} & =-\frac{2}{M_{A_{0}}^{2}} \Sigma^{G_{0} A_{0}}\left(M_{A_{0}}^{2}\right), \\
\delta Z_{A_{0} G_{0}} & =\frac{2}{M_{A_{0}}^{2}\left(M^{2}-M_{A_{0}}^{2}\right)}\left(M^{2} \Sigma^{G_{0} A_{0}}\left(M_{A_{0}}^{2}\right)-M_{A_{0}}^{2} \Sigma^{G_{0} A_{0}}\left(M^{2}\right)\right),
\end{aligned}
$$


where $M^{2}$ is an arbitrary scale at which the $G_{0} A_{0}$ mixing energy $\Sigma^{G_{0} A_{0}}$ is required to vanish. Choosing, for instance, $M=0$ leads to

$$
\delta Z_{A_{0} G_{0}}=\frac{2}{M_{A_{0}}^{2}} \Sigma^{G_{0} A_{0}}(0)
$$

and [in analogy to (3.41)]

$$
\delta \beta=\frac{1}{4}\left(\delta Z_{G_{0} A_{0}}-\delta Z_{A_{0} G_{0}}\right)=-\frac{1}{2 M_{A_{0}}^{2}}\left(\Sigma^{G_{0} A_{0}}\left(M_{A_{0}}^{2}\right)+\Sigma^{G_{0} A_{0}}(0)\right) .
$$

In the BFM (see section 3.3.3) this simplifies owing to the Ward identity ${ }^{13}$ (E.27), $\Sigma \hat{G}_{0} \hat{A}_{0}(0)=0$, to

$$
\delta \beta=\frac{1}{4}\left(\delta Z_{\hat{G}_{0} \hat{A}_{0}}-\delta Z_{\hat{A}_{0} \hat{G}_{0}}\right)=-\frac{1}{2 M_{A_{0}}^{2}} \Sigma^{\hat{G}_{0} \hat{A}_{0}}\left(M_{A_{0}}^{2}\right) .
$$

This expression formally coincides with the renormalization of $\beta$ proposed in ref. [23] within the Landau gauge of the conventional formalism. We stress that the simple form (3.50) for the counterterm $\delta \beta$ holds only in special gauges, while in general (3.49) has to be used. Moreover, (3.50) requires the PRTS scheme of ref. [9], while in the FJTS scheme extra tadpole contributions appear [see eq. (3.82) below].

\subsubsection{Background-field gauge invariance}

The method of the previous sections is not applicable to the renormalization of parameters that are not directly related to the mixing of fields. An example is the renormalization of the singlet sector of the HSESM. Besides the mass of the second Higgs scalar, a second parameter has to be selected. One can choose one of the quartic couplings $\lambda_{1}$ or $\lambda_{3}$ of the Higgs-singlet field, its vev $v_{1}$, or the quantity $\tan \beta=v_{2} / v_{1}$, in analogy to the THDM. In such cases, the background-field gauge invariance that appears when quantizing in the BFM can be exploited to fix renormalization constants. The BFM (see, e.g., refs. [54-56]) for the EW SM was introduced in ref. [34], and its application to the THDM and the HSESM was described in ref. [28]. Following these references we denote background fields with carets. All relations discussed in sections 3.3.1 and 3.3.2 hold in the BFM for the corresponding quantities of background fields as well.

In the conventional formalism, the gauge-fixing term breaks rigid invariance. ${ }^{14}$ In the $\mathrm{BFM}$, rigid gauge invariance is maintained for the background fields upon choosing an appropriate gauge-fixing term for the quantum fields [28, 34]. Rigid invariance for the background field gives rise to Ward identities (cf. appendix E) and restrictions on the renormalization constants for the background fields. In particular, in the BFM the relations (3.37)-(3.40) can all be maintained including the finite parts. The relations resulting from rigid gauge invariance in the BFM were presented for the SM in the renormalization

\footnotetext{
${ }^{13}$ For the validity of (E.27) it is crucial that the self-energy is the quantity appearing in the inverse of the propagator, i.e. that it includes tadpole contributions and corresponding counterterms.

${ }^{14}$ This does not affect the discussion in sections 3.3.1 and 3.3.2, since it relies only on the fact that a symmetric field renormalization is possible in the spontaneously broken phase.
} 
scheme of ref. [9] in ref. [34]. In the FJTS scheme, as defined in refs. [27, 31], additional tadpole contributions $\propto \Delta v$ appear in the Ward identities and thus also in these relations. For instance, using the conventions of ref. [28] the last line of eq. (46) of ref. [34] for the $\mathrm{SM}$ in the FJTS scheme becomes

$$
\delta Z_{\hat{\Phi}}=\delta Z_{\hat{\eta}}=\delta Z_{\hat{\chi}}=\delta Z_{\hat{\phi}}=-2 \delta Z_{e}-\frac{c_{\mathrm{w}}^{2}}{s_{\mathrm{w}}^{2}} \frac{\delta c_{\mathrm{w}}^{2}}{c_{\mathrm{w}}^{2}}+\frac{\delta M_{\mathrm{W}}^{2}}{M_{\mathrm{W}}^{2}}+2 \frac{\Delta v}{v},
$$

where the extra term, the shift $\Delta v$ in the vev, is related to the tadpole by

$$
\Delta v=\frac{-\delta t_{\hat{H}}}{M_{\mathrm{H}}^{2}}=\frac{T^{\hat{H}}}{M_{\mathrm{H}}^{2}},
$$

$\delta t_{\hat{H}}$ denotes the tadpole counterterm and $T^{\hat{H}}$ the unrenormalized tadpole, i.e. the renormalized Higgs one-point vertex function is given by $\Gamma^{\hat{H}}=\left(T^{\hat{H}}+\delta t_{\hat{H}}\right)=0$. The counterterms $\delta Z_{e}$ and $\delta c_{\mathrm{w}}^{2}$ are fixed according to (48) and (46) of ref. [34] and can be shown to be independent of the tadpole counterterm scheme. In the FJTS scheme the W-boson mass counterterm gets additional implicit tadpole contributions $\propto \Delta v$ as described in ref. [27] which is equivalent to including explicit tadpoles in the self-energy that determines the counterterm, i.e.

$$
\delta M_{\mathrm{W}}^{2}=\Sigma_{\mathrm{T}}^{\hat{W} \hat{W}}\left(M_{\mathrm{W}}^{2}\right)=\Sigma_{1 \mathrm{PI}, \mathrm{T}}^{\hat{W}}\left(M_{\mathrm{W}}^{2}\right)-\frac{e M_{\mathrm{W}}}{s_{\mathrm{w}}} \Delta v=\Sigma_{1 \mathrm{PI}, \mathrm{T}}^{\hat{W}}\left(M_{\mathrm{W}}^{2}\right)-\frac{e M_{\mathrm{W}}}{s_{\mathrm{W}} M_{\mathrm{H}}^{2}} T^{\hat{H}} .
$$

As a consequence, in the FJTS scheme the W-boson mass counterterm is gauge independent, and the gauge dependence of $\delta Z_{\hat{\eta}}$ matches the one of $\Delta v$. In the renormalization scheme of ref. [9] the tadpole contribution in (3.53) is absent, and the mass counterterm is gauge dependent (since the definition of the bare mass involves the shifted vev, see the discussion in ref. [28]).

Using

$$
M_{\mathrm{W}}=\frac{e}{2 s_{\mathrm{w}}} v, \quad M_{\mathrm{W}, \mathrm{B}}=\frac{e_{\mathrm{B}}}{2 s_{\mathrm{w}, \mathrm{B}}} v_{\mathrm{B}}
$$

the definition

$$
v_{\mathrm{B}}=Z_{v} v=v\left(1+\delta Z_{v}\right),
$$

as well as the renormalization transformations of the SM parameters, the relation (3.51) implies

$$
\delta Z_{\hat{\Phi}}=\delta Z_{\hat{\eta}}=\delta Z_{\hat{\chi}}=\delta Z_{\hat{\phi}}=2 \delta Z_{v}+2 \frac{\Delta v}{v} .
$$

Thus, the vev is renormalized as the corresponding scalar doublet field apart from the explicit shift $\Delta v$ introduced to ensure a vanishing tadpole at one-loop order.

In the rest of this section we use the FJTS scheme.

Higgs singlet extension of the Standard Model. In the HSESM within the BFM, (3.51) and (3.56) still hold with the components of the SM doublet replaced by those of the doublet of this model,

$$
\delta Z_{\hat{\Phi}}=\delta Z_{2}^{\hat{\eta}}=\delta Z_{\hat{\eta}_{2}}=\delta Z_{\hat{\chi}}=\delta Z_{\hat{\phi}}=2 \delta Z_{v_{2}}+2 \frac{\Delta v_{2}}{v_{2}}
$$


where

$$
\delta Z_{v_{2}}=-\delta Z_{e}-\frac{1}{2} \frac{c_{\mathrm{w}}^{2}}{s_{\mathrm{w}}^{2}} \frac{\delta c_{\mathrm{w}}^{2}}{c_{\mathrm{w}}^{2}}+\frac{1}{2} \frac{\delta M_{\mathrm{W}}^{2}}{M_{\mathrm{W}}^{2}} .
$$

In addition, we obtain a corresponding relation between the renormalization of the component $\eta_{1}$ of the singlet field $\sigma$ and the corresponding vev $v_{1}$,

$$
\delta Z_{\hat{\sigma}}=\delta Z_{1}^{\hat{\eta}}=\delta Z_{\hat{\eta}_{1}}=2 \delta Z_{v_{1}}+2 \frac{\Delta v_{1}}{v_{1}} .
$$

The shifts of the vevs can be expressed in terms of the tadpole counterterms as

$$
\Delta v_{1}=-\frac{\delta t_{\hat{H}_{1}}}{M_{\mathrm{H}_{1}}^{2}} c_{\alpha}+\frac{\delta t_{\hat{H}_{2}}}{M_{\mathrm{H}_{2}}^{2}} s_{\alpha}, \quad \Delta v_{2}=-\frac{\delta t_{\hat{H}_{1}}}{M_{\mathrm{H}_{1}}^{2}} s_{\alpha}-\frac{\delta t_{\hat{H}_{2}}}{M_{\mathrm{H}_{2}}^{2}} c_{\alpha}
$$

Fixing $\delta Z_{2}^{\hat{\eta}}$ from (3.57), using the on-shell field renormalization constants (2.5) to determine $\delta Z_{1}^{\hat{\eta}}$ from (3.39) including the finite parts,

$$
\delta Z_{12}^{H}+\delta Z_{21}^{H}=2 c_{\alpha} s_{\alpha}\left(\delta Z_{2}^{\eta}-\delta Z_{1}^{\eta}\right)
$$

where $\delta Z_{i j}^{\hat{H}}$ are the elements of the field renormalization matrix for the scalars as defined in (2.4), we can use (3.59) to fix the renormalization of the singlet vev. This results in

$$
\begin{aligned}
\delta Z_{v_{1}} & =\frac{1}{2}\left(\delta Z_{1}^{\hat{\eta}}-\delta Z_{2}^{\hat{\eta}}\right)+\delta Z_{v_{2}}+\frac{\Delta v_{2}}{v_{2}}-\frac{\Delta v_{1}}{v_{1}} \\
& =-\frac{1}{4 c_{\alpha} s_{\alpha}}\left(\delta Z_{12}^{\hat{H}}+\delta Z_{21}^{\hat{H}}\right)+\delta Z_{v_{2}}+\frac{\Delta v_{2}}{v_{2}}-\frac{\Delta v_{1}}{v_{1}} .
\end{aligned}
$$

Defining

$$
\tan \beta_{\mathrm{B}}=\frac{v_{2, \mathrm{~B}}}{v_{1, \mathrm{~B}}}, \quad \tan \beta=\frac{v_{2}}{v_{1}}
$$

this translates to

$$
\begin{aligned}
\delta \tan \beta & =\frac{1}{2} \tan \beta\left(\delta Z_{2}^{\hat{\eta}}-\delta Z_{1}^{\hat{\eta}}+2 \frac{\Delta v_{1}}{v_{1}}-2 \frac{\Delta v_{2}}{v_{2}}\right) \\
& =\frac{1}{4} \frac{\tan \beta}{c_{\alpha} s_{\alpha}}\left(\delta Z_{12}^{\hat{H}}+\delta Z_{21}^{\hat{H}}\right)+\tan \beta\left(\frac{\Delta v_{1}}{v_{1}}-\frac{\Delta v_{2}}{v_{2}}\right),
\end{aligned}
$$

where

$$
\frac{\Delta v_{1}}{v_{1}}-\frac{\Delta v_{2}}{v_{2}}=\frac{e}{2 s_{\mathrm{W}} M_{\mathrm{W}}}\left[\frac{\delta t_{\hat{H}_{1}}}{M_{\mathrm{H}_{1}}^{2}}\left(s_{\alpha}-c_{\alpha} \tan \beta\right)+\frac{\delta t_{\hat{H}_{2}}}{M_{\mathrm{H}_{2}}^{2}}\left(c_{\alpha}+s_{\alpha} \tan \beta\right)\right] .
$$

Alternative definitions of the counterterms can be obtained upon using (3.37) and (3.38) instead of (3.39) to fix $\left(\delta Z_{1}^{\hat{\eta}}-\delta Z_{2}^{\hat{\eta}}\right)$. In particular, upon using an appropriate linear combination of (3.37), (3.38) and (3.39), one finds

$$
\delta Z_{1}^{\hat{\eta}}-\delta Z_{2}^{\hat{\eta}}=\left(c_{\alpha}^{2}-s_{\alpha}^{2}\right)\left(\delta Z_{11}^{\hat{H}}-\delta Z_{22}^{\hat{H}}\right)-2 s_{\alpha} c_{\alpha}\left(\delta Z_{12}^{\hat{H}}+\delta Z_{21}^{\hat{H}}\right)
$$


Using this expression avoids the potential singularity for $c_{\alpha} \rightarrow 0$ or $s_{\alpha} \rightarrow 0$ in (3.64) and leads to

$$
\delta Z_{v_{1}}=\frac{1}{2}\left[\left(c_{\alpha}^{2}-s_{\alpha}^{2}\right)\left(\delta Z_{11}^{\hat{H}}-\delta Z_{22}^{\hat{H}}\right)-2 s_{\alpha} c_{\alpha}\left(\delta Z_{12}^{\hat{H}}+\delta Z_{21}^{\hat{H}}\right)\right]+\delta Z_{v_{2}}+\frac{\Delta v_{2}}{v_{2}}-\frac{\Delta v_{1}}{v_{1}}
$$

or

$$
\begin{aligned}
\delta \tan \beta= & \frac{1}{2} \tan \beta\left[\left(s_{\alpha}^{2}-c_{\alpha}^{2}\right)\left(\delta Z_{11}^{\hat{H}}-\delta Z_{22}^{\hat{H}}\right)+2 s_{\alpha} c_{\alpha}\left(\delta Z_{12}^{\hat{H}}+\delta Z_{21}^{\hat{H}}\right)\right] \\
& +\tan \beta\left(\frac{\Delta v_{1}}{v_{1}}-\frac{\Delta v_{2}}{v_{2}}\right)
\end{aligned}
$$

instead of (3.62) and (3.64).

Equations (3.62)-(3.68) can be used to fix the renormalization of $v_{1}$ or $\beta$ upon using the field renormalization constants $\delta Z_{i j}^{H}$ for the scalar fields (2.5) in the complete on-shell scheme. Since these are gauge dependent, a gauge needs to be fixed. A convenient choice is the 't Hooft-Feynman gauge of the BFM. The renormalization scheme based on (3.68) and (3.65) for $\beta$ and on (3.41) for $\alpha$ in the HSESM is denoted as BFMS in the following. ${ }^{15}$

Two-Higgs-doublet model. Considering now the THDM and using again the BFM and the corresponding background-field gauge invariance, we obtain instead of (3.51):

$$
\begin{aligned}
& \delta Z_{1}^{\hat{\eta}}=-2 \delta Z_{e}-\frac{c_{\mathrm{w}}^{2}}{s_{\mathrm{w}}^{2}} \frac{\delta c_{\mathrm{w}}^{2}}{c_{\mathrm{w}}^{2}}+\frac{\delta M_{\mathrm{W}}^{2}}{M_{\mathrm{W}}^{2}}+2 \frac{\Delta v_{1}}{v_{1}}+2 \frac{\delta c_{\beta}}{c_{\beta}}, \\
& \delta Z_{2}^{\hat{\eta}}=-2 \delta Z_{e}-\frac{c_{\mathrm{w}}^{2}}{s_{\mathrm{w}}^{2}} \frac{\delta c_{\mathrm{w}}^{2}}{c_{\mathrm{w}}^{2}}+\frac{\delta M_{\mathrm{W}}^{2}}{M_{\mathrm{W}}^{2}}+2 \frac{\Delta v_{2}}{v_{2}}+2 \frac{\delta s_{\beta}}{s_{\beta}},
\end{aligned}
$$

where the shifts of the vevs can be expressed via the tadpole counterterms as

$$
\Delta v_{1}=-\frac{\delta t_{\hat{H}_{1}}}{M_{\mathrm{H}_{1}}^{2}} c_{\alpha}+\frac{\delta t_{\hat{H}_{2}}}{M_{\mathrm{H}_{2}}^{2}} s_{\alpha}, \quad \Delta v_{2}=-\frac{\delta t_{\hat{H}_{1}}}{M_{\mathrm{H}_{1}}^{2}} s_{\alpha}-\frac{\delta t_{\hat{H}_{2}}}{M_{\mathrm{H}_{2}}^{2}} c_{\alpha} .
$$

Equations (3.69) and (3.70) imply

$$
\begin{gathered}
\delta \beta=\frac{1}{2} c_{\beta} s_{\beta}\left(\delta Z_{2}^{\hat{\eta}}-\delta Z_{1}^{\hat{\eta}}\right)+\frac{e}{2 s_{\mathrm{w}} M_{\mathrm{W}}}\left(s_{\beta} \Delta v_{1}-c_{\beta} \Delta v_{2}\right), \\
c_{\beta}^{2} \delta Z_{1}^{\hat{\eta}}+s_{\beta}^{2} \delta Z_{2}^{\hat{\eta}}=-2 \delta Z_{e}-\frac{c_{\mathrm{w}}^{2}}{s_{\mathrm{w}}^{2}} \frac{\delta c_{\mathrm{w}}^{2}}{c_{\mathrm{w}}^{2}}+\frac{\delta M_{\mathrm{W}}^{2}}{M_{\mathrm{W}}^{2}}+\frac{e}{s_{\mathrm{w}} M_{\mathrm{W}}}\left(c_{\beta} \Delta v_{1}+s_{\beta} \Delta v_{2}\right) .
\end{gathered}
$$

Using (3.39) including finite parts, this yields

$$
\delta \beta=\frac{1}{4} \frac{c_{\beta} s_{\beta}}{c_{\alpha} s_{\alpha}}\left(\delta Z_{12}^{\hat{H}}+\delta Z_{21}^{\hat{H}}\right)+\frac{e}{2 s_{\mathrm{w}} M_{\mathrm{W}}}\left(s_{\beta} \Delta v_{1}-c_{\beta} \Delta v_{2}\right) .
$$

The terms involving shifts in the vevs in (3.74) can be expressed by the tadpoles as

$$
s_{\beta} \Delta v_{1}-c_{\beta} \Delta v_{2}=\frac{\delta t_{\hat{H}_{1}}}{M_{\mathrm{H}_{1}}^{2}} \sin (\alpha-\beta)+\frac{\delta t_{\hat{H}_{2}}}{M_{\mathrm{H}_{2}}^{2}} \cos (\alpha-\beta) .
$$

\footnotetext{
${ }^{15}$ Note that in the first preprint version of this paper the scheme BFMS in the HSESM was based on (3.64) and (3.65). For the scenarios considered in section 4 the differences between the two choices in the numerical results are marginal.
} 
As for the HSESM, alternative counterterms can be obtained using (3.37) and (3.38) instead of (3.39) to fix $\left(\delta Z_{1}^{\hat{\eta}}-\delta Z_{2}^{\hat{\eta}}\right)$. If $s_{\alpha}$ or $c_{\alpha}$ become small, the counterterm defined by (3.74) becomes artificially large, which is actually the case in the THDM scenarios B1 and B2 considered in section 4 below. This can be avoided by using (3.66) in eq. (3.72), resulting in

$$
\begin{aligned}
\delta \beta= & \frac{1}{2} c_{\beta} s_{\beta}\left[\left(s_{\alpha}^{2}-c_{\alpha}^{2}\right)\left(\delta Z_{11}^{\hat{H}}-\delta Z_{22}^{\hat{H}}\right)+2 c_{\alpha} s_{\alpha}\left(\delta Z_{12}^{\hat{H}}+\delta Z_{21}^{\hat{H}}\right)\right] \\
& +\frac{e}{2 s_{\mathrm{W}} M_{\mathrm{W}}}\left(s_{\beta} \Delta v_{1}-c_{\beta} \Delta v_{2}\right)
\end{aligned}
$$

The renormalization scheme based on (3.76) and (3.75) for $\beta$ and (3.41) within the BFM for $\alpha$ in the THDM is denoted as BFMS in the following. ${ }^{16}$ We note that the renormalization of $\alpha$ in the "on-shell tadpole-pinched scheme" of ref. [26] is equivalent to the one based on (3.41) within the BFM.

There are a number of further possibilities to fix the counterterm $\delta \beta$ using rigid invariance in the BFM. The discussion of sections 3.3.1 and 3.3.2 holds also for the mixing between the pseudoscalar or charged scalar fields. This gives rise to the relations

$$
\begin{aligned}
\delta Z_{11} & =c_{\beta}^{2} \delta Z_{1}^{\hat{\eta}}+s_{\beta}^{2} \delta Z_{2}^{\hat{\eta}}, \\
\delta Z_{22} & =s_{\beta}^{2} \delta Z_{1}^{\hat{\eta}}+c_{\beta}^{2} \delta Z_{2}^{\hat{\eta}}, \\
\delta Z_{12}+\delta Z_{21} & =2 c_{\beta} s_{\beta}\left(\delta Z_{2}^{\hat{\eta}}-\delta Z_{1}^{\hat{\eta}}\right), \\
\delta Z_{12}-\delta Z_{21} & =4 \delta \beta,
\end{aligned}
$$

where $\delta Z_{i j}$ refer to the field renormalization constants of the pseudoscalar $\left(G_{0}, A_{0}\right)^{\mathrm{T}}$ or of the charged scalar $\left(G^{ \pm}, H^{ \pm}\right)^{\mathrm{T}}$ fields.

From eqs. (3.72), (3.73), (3.77)-(3.80) we can derive

$$
\begin{aligned}
& \delta Z_{11}=-2 \delta Z_{e}-\frac{c_{\mathrm{w}}^{2}}{s_{\mathrm{w}}^{2}} \frac{\delta c_{\mathrm{w}}^{2}}{c_{\mathrm{w}}^{2}}+\frac{\delta M_{\mathrm{W}}^{2}}{M_{\mathrm{W}}^{2}}+\frac{e}{s_{\mathrm{w}} M_{\mathrm{W}}}\left(c_{\beta} \Delta v_{1}+s_{\beta} \Delta v_{2}\right), \\
& \delta Z_{21}=\frac{e}{s_{\mathrm{w}} M_{\mathrm{W}}}\left(c_{\beta} \Delta v_{2}-s_{\beta} \Delta v_{1}\right),
\end{aligned}
$$

as well as the relations for the counterterm to the mixing angle $\beta$

$$
\begin{aligned}
& \delta \beta=\frac{1}{4}\left(\delta Z_{12}+\delta Z_{21}\right)+\frac{e}{2 s_{\mathrm{W}} M_{\mathrm{W}}}\left(s_{\beta} \Delta v_{1}-c_{\beta} \Delta v_{2}\right), \\
& \delta \beta=\frac{1}{4} \delta Z_{12}+\frac{e}{4 s_{\mathrm{W}} M_{\mathrm{W}}}\left(s_{\beta} \Delta v_{1}-c_{\beta} \Delta v_{2}\right),
\end{aligned}
$$

where we can use the field renormalization constants of either $\left(G_{0}, A_{0}\right)^{\mathrm{T}}$ or $\left(G^{ \pm}, H^{ \pm}\right)^{\mathrm{T}}$ in the complete on-shell scheme. Note that (3.82) results from (2.5) and the Ward identity (E.31) valid in the FJTS scheme.

Equations (3.74), (3.83), or (3.84) provide all the same divergent parts for $\delta \beta$, but differ in the finite parts. Moreover, all conditions are gauge dependent, since the on-shell

\footnotetext{
${ }^{16}$ Note that in the first preprint version of this paper the scheme BFMS in the THDM was based on eq. (3.74) and (3.75).
} 


\begin{tabular}{|c|c|c|c|}
\hline Scheme & $\alpha$ & $\lambda_{1}, v_{1}$, or $\tan \beta$ & comments \\
\hline$\overline{\mathrm{MS}}(\mathrm{PRTS})$ & $\overline{\mathrm{MS}}$ & $\overline{\mathrm{MS}}$ for $\lambda_{1}$ & no tadpoles in mass terms \\
\hline$\overline{\mathrm{MS}}$ (FJTS) & $\overline{\mathrm{MS}}$ & $\overline{\mathrm{MS}}$ for $\lambda_{1}$ & FJ Tadpole Scheme \\
\hline OS & $(3.13)$ & $\overline{\mathrm{MS}}$ for $\lambda_{1}$ & on-shell renormalization of $\alpha$ \\
\hline BFMS & $(3.41)$ & $\begin{array}{c}v_{1} \text { via }(3.62), \\
\text { or equivalently } \tan \beta \text { via }(3.64)\end{array}$ & $\Sigma_{12}^{\hat{H}}, T^{\hat{H}_{i}}$ for $\delta \alpha$ from BFM \\
\hline
\end{tabular}

Table 1. Summary of renormalization schemes used in the HSESM.

\begin{tabular}{|c|c|c|c|c|}
\hline Scheme & $\alpha$ & $\beta$ & $\lambda_{5}$ & comments \\
\hline$\overline{\mathrm{MS}}$ (PRTS) & $\overline{\mathrm{MS}}$ & $\overline{\mathrm{MS}}$ & $\overline{\mathrm{MS}}$ & no tadpoles in mass terms; $\overline{\mathrm{MS}}(\alpha)$ in ref. [29] \\
\hline$\overline{\mathrm{MS}}$ (FJTS) & $\overline{\mathrm{MS}}$ & $\overline{\mathrm{MS}}$ & $\overline{\mathrm{MS}}$ & FJ Tadpole Scheme; FJ $(\alpha)$ in ref. [29] \\
\hline OS1 & $(3.17)$ & $(3.21)$ & $\overline{\mathrm{MS}}$ & on-shell renormalization of $\alpha$ and $\beta$ \\
\hline OS2 & $(3.18)$ & $(3.24)$ & $\overline{\mathrm{MS}}$ & on-shell renormalization of $\alpha$ and $\beta$ \\
\hline OS12 & $(3.18)$ & $(3.30)$ & $\overline{\mathrm{MS}}$ & on-shell renormalization of $\alpha$ and $\beta$ \\
\hline BFMS & $(3.41)$ & $(3.74)$ & $\overline{\mathrm{MS}}$ & $\Sigma_{12}^{\hat{H}}, T^{\hat{H}_{i}}$ for $\delta \alpha$ and $\delta \beta$ from BFM \\
\hline
\end{tabular}

Table 2. Summary of renormalization schemes used in the THDM.

field renormalization constants are gauge dependent. Specific renormalization schemes can be fixed upon choosing a specific gauge, such as the 't Hooft-Feynman gauge (within the $\mathrm{BFM})$ and a specific equation.

For the gauge dependence of the renormalization schemes based on the BFM the same remarks as in section 3.3.1 apply.

\subsection{Summary of renormalization schemes}

The various renormalization schemes used for the HSESM in this paper are summarized in table 1. The $\overline{\mathrm{MS}}$ and FJ schemes discussed in ref. [37] are identical to the $\overline{\mathrm{MS}}$ (PRTS) and $\overline{\mathrm{MS}}$ (FJTS) schemes of this paper, up to the point that $\lambda_{12}^{[37]}=\lambda_{3} / 2$ is used as $\overline{\mathrm{MS}}$ renormalized parameter instead of $\lambda_{1}$. The $\overline{\mathrm{MS}}$ scheme of ref. [28] is identical to the $\overline{\mathrm{MS}}$ (FJTS) scheme of this paper.

The renormalization schemes used for the THDM in this paper are summarized in table 2. The $\overline{\mathrm{MS}}\left(\lambda_{3}\right)$ and $\mathrm{FJ}\left(\lambda_{3}\right)$ schemes of ref. [29], where the scalar coupling $\lambda_{3}$ replaces the angle $\alpha$ as $\overline{\mathrm{MS}}$-renormalized parameter of the $\overline{\mathrm{MS}}(\alpha)$ and $\mathrm{FJ}(\alpha)$ schemes, are not considered in this paper. The $\overline{\mathrm{MS}}$ scheme of ref. [27] coincides with the $\overline{\mathrm{MS}}$ (FJTS) scheme of this paper up to the fact that $\lambda_{5}$ is used as $\overline{\mathrm{MS}}$-renormalized parameter instead of $M_{\mathrm{sb}}^{2}=M_{A_{0}}^{2}+4 M_{\mathrm{W}}^{2} s_{\mathrm{w}}^{2} \lambda_{5} / e^{2}$.

\subsection{Parameter conversion between renormalization schemes}

\subsubsection{Matching procedure and running couplings}

For a comparison of predictions based on different renormalization schemes a conversion of renormalized parameters is necessary. The matching between different schemes is based on 
the fact that the bare parameters defining the model are renormalization-scheme independent. Following ref. [29], we describe two variants that can be used to convert renormalized parameters at NLO.

Denoting a set of input parameters generically as $\left\{p_{i}\right\}$ defined in two different renormalization schemes "(1)" and "(2)", the two different renormalization schemes are connected via

$$
p_{\mathrm{B}, i}=p_{i}^{(1)}+\delta p_{i}^{(1)}\left(\left\{p_{j}^{(1)}\right\}\right)=p_{i}^{(2)}+\delta p_{i}^{(2)}\left(\left\{p_{j}^{(2)}\right\}\right),
$$

where $\left\{p_{\mathrm{B}, i}\right\}$ is the set of bare parameters which are by definition renormalization-scheme independent. There are basically two possibilities to translate the renormalized parameters from scheme (1) into (2): performing a "full conversion" upon solving (3.85) numerically for $\left\{p_{j}^{(2)}\right\}$ with a given set of parameters $\left\{p_{j}^{(1)}\right\}$, or linearizing (3.85) in $p_{i}^{(2)}$ by replacing $\left\{p_{j}^{(2)}\right\}$ by $\left\{p_{j}^{(1)}\right\}$ in the last term, so that

$$
p_{i}^{(2)}=p_{i}^{(1)}+\delta p_{i}^{(1)}\left(\left\{p_{j}^{(1)}\right\}\right)-\delta p_{i}^{(2)}\left(\left\{p_{j}^{(1)}\right\}\right)+\ldots,
$$

which is valid up to terms beyond NLO. The results of the two versions agree in NLO accuracy. The advantage of the full conversion is mainly the exact invertibility, i.e. converting from scheme (1) into (2) and back into (1), reproduces the parameters $\left\{p_{j}^{(1)}\right\}$ exactly, while the linearized version reproduces those parameters only in NLO accuracy.

In the subsequent section, we make use of the full conversion to translate benchmark scenarios between the various renormalization schemes. For the THDM, this means that up to three parameters are converted at a time; for the HSESM, only up to two parameters are concerned. For each benchmark scenario we perform the conversion at a chosen central scale $\mu=\mu_{0}$, where $\mu_{0}$ is chosen according to the process and model. In the extension of Prophecy4F [57, 58] to the HSESM [37] and THDM [29, 41], which is used to calculate Higgs decay widths into four fermions at NLO, (3.85) is solved numerically in double precision by minimizing the $\chi^{2}$ of the error when solving

$$
p_{i}^{(2)}=p_{i}^{(1)}+\delta p_{i}^{(1)}\left(\left\{p_{j}^{(1)}\right\}\right)-\delta p_{i}^{(2)}\left(\left\{p_{j}^{(2)}\right\}\right)
$$

for $\left\{p_{j}^{(2)}\right\}$ by iteration. ${ }^{17}$ In RECOLA2, quasi-Newton methods (based on the L-BFGS algorithm) are used to find the full solution compatible in double precision. This procedure is used to prepare run cards for the Monte Carlo program HAWK 2.0 [59], with the parameters already converted from a specific input scheme and evolved to the scale, which are then used to evaluate Higgs-production cross sections at NLO.

In order discuss scale uncertainties, we perform the scale variation by including effects of running $\overline{\mathrm{MS}}$-renormalized parameters. The running is given by the following coupled system of differential equations

$$
\frac{\partial}{\partial \mu^{2}} p_{i}\left(\mu^{2}\right)=\beta_{p_{i}}\left(\left\{p_{j}\left(\mu^{2}\right)\right\}\right),
$$

\footnotetext{
${ }^{17}$ For the mixing angles $\alpha$ and $\beta$, the matching equation is written in the (NLO-correct) form $\alpha^{\overline{\mathrm{MS}(\mathrm{PRTS})}}=$ $\alpha^{\overline{\mathrm{MS}}(\mathrm{FJTS})}-\left.\Delta \alpha^{t}\left(T^{H_{1}}, T^{H_{2}}\right)\right|_{\text {finite }}$ with finite tadpole terms quantified by the function $\Delta \alpha^{t}$, and similarly for $\beta$.
} 
where $\beta_{p_{i}}$ denotes the $\beta$-function of $p_{i}$ which is non-zero if $p_{i}$ is defined in an $\overline{\mathrm{MS}}$ scheme. For the integration we use standard Runge-Kutta techniques.

\subsubsection{Higher-order ambiguities in the conversion}

The "full" parameter conversion described in the previous section suffers from ambiguities that are connected to higher-order contributions beyond NLO accuracy. Firstly, the whole renormalization programs addressed in this paper are worked out to NLO, i.e. many relations between renormalizations constants are worked out to linear order only. Beyond NLO, many missing terms should be completed. Secondly, taking the matching equation (3.85) at NLO (or any fixed order) in the "full" conversion, it should be noted that not all UV divergences cancel exactly, because the parameters in the coefficients in front of the UVdivergent contributions in $\delta p^{(i)}$ are not the same (but defined in different schemes). That means that some UV scale has to be fixed in the evaluation of $\delta p^{(i)}$ (which is typically set to the renormalization scale), the effect of which, however, is beyond NLO.

We, thus, cannot claim that the full conversion is more precise than the linearized version; both are equivalent at NLO. As already mentioned, the full conversion has the advantage of being exactly invertible. Another benefit of supporting both versions is the fact that the comparison of the respective results gives the typical size of effects beyond NLO, which often helps to identify scenarios which are perturbatively unstable.

Looking beyond NLO, it should be mentioned that again specific care has to be taken with respect to the inclusion of tadpole contributions in the matching equation (3.85). For instance, in the PRTS scheme tadpole contributions enter the relations between bare parameters, while in the FJTS scheme they do not. Thus, if the matching of the schemes is done in the original parametrization of the theory (i.e. at the level of $\mu^{2}$ and $\lambda$ parameters in the scalar potential), in general there will be tadpole contributions in $\delta p_{i}^{(\mathrm{PRTS})}$, but not in $\delta p_{i}^{(\mathrm{FJTS})}$, so that

$$
\delta p_{i}^{(\mathrm{FJTS})}=\delta p_{i}^{(\mathrm{PRTS})}+\Delta T_{i}\left(\delta t_{j}^{(\mathrm{PRTS})}\right)
$$

where $\Delta T_{i}\left(\delta t_{j}^{\text {(PRTS })}\right)$ is some function of PRTS tadpole counterterms $\delta t_{j}^{\mathrm{PRTS}}$.

Besides the treatment of tadpoles other sources of ambiguities exist. Since the conversion is performed at fixed loop order the precise choice of the independent parameters matters in higher orders. For instance, the matching of bare parameters for the mixing angles can be performed directly on the mixing angles

$$
\alpha_{\mathrm{B}}=\alpha+\delta \alpha, \quad \beta_{\mathrm{B}}=\beta+\delta \beta,
$$

or it can be performed on derived parameters such as

$$
\tan \alpha_{\mathrm{B}}=\tan \alpha+\delta \tan \alpha, \quad \tan \beta_{\mathrm{B}}=\tan \beta+\delta \tan \beta,
$$

which will result in a conversion equivalent only at NLO. In appendix $\mathrm{F}$ the conversion tables for the input parameters of section 4 are given. The conversion is performed in two variants which exemplify the before-mentioned ambiguities and their impact on final results. 


\section{Phenomenological results}

\subsection{Higgs-boson decays $\mathrm{H}_{1,2} \rightarrow \mathrm{WW} / \mathrm{ZZ} \rightarrow 4$ fermions}

The Monte Carlo program Prophecy4F [57, 58] provides a "PROPer description of the Higgs dECaY into 4 Fermions" and calculates observables for the decay process Higgs $\rightarrow \mathrm{WW} / \mathrm{ZZ} \rightarrow 4$ fermions at NLO EW+QCD. Its first version [57, 58] was designed for the SM and slightly generalized to include a possible fourth fermion generation in ref. [60]. In refs. [29, 41] and [37], PropheCy4F was extended to the corresponding decays of the light CP-even Higgs bosons of the THDM and the HSESM, respectively, keeping the functionality and applicability of the program basically the same. The THDM and the HSESM were renormalized using $\overline{\mathrm{MS}}$ schemes for the mixing angles $\alpha$ and $\beta$. In the following, we present first results from a further extension of PROPHECY $4 \mathrm{~F}^{18}$ which covers the decays of the heavy CP-even scalar bosons as well and which additionally supports the on-shell and symmetry-inspired renormalization schemes described in this paper. All Higgs-boson decays via intermediate on- or off-shell EW gauge bosons W/Z into all light fermions (all other than top quarks) are supported, but potential decays of a heavy Higgs boson into a pair of light Higgs bosons such as $\mathrm{H}_{1} \rightarrow \mathrm{H}_{2} \mathrm{H}_{2}$, or to t $\overline{\mathrm{t}}$ pairs are considered as separate processes and not included in the calculation.

\subsubsection{Higgs-singlet extension of the Standard Model}

In ref. [37], the HSESM was renormalized in two schemes, called $\overline{\mathrm{MS}}$ and FJ there, which are identical with the $\overline{\mathrm{MS}}$ (PRTS) and $\overline{\mathrm{MS}}$ (FJTS) schemes of this paper up to the point that different $\overline{\mathrm{MS}}$-renormalized quartic scalar couplings $\lambda_{i}$ are used to parametrize the Higgs sector. In ref. [37], the coupling $\lambda_{12}=\lambda_{3} / 2$, which mixes the doublet and singlet scalars was chosen, while we choose the quartic coupling $\lambda_{1}$ of the singlet sector in this paper instead. For the application to the decays $\mathrm{H}_{1,2} \rightarrow 4 f$, this choice makes only a marginal difference, which is even beyond NLO, since the renormalization of those quartic Higgs couplings does not enter in this case. Only a minor effect from the different running of the couplings in some one-loop corrections remains.

In ref. [37], five different HSESM scenarios were considered, which are still compatible with current LHC results. These scenarios, which are called BHM200 ${ }^{ \pm}$BHM400, BHM600, and BHM800, identify the light Higgs boson $\mathrm{H}_{2}$ with the discovered state with a mass of $\sim 125 \mathrm{GeV}$ and contain a heavy Higgs boson $\mathrm{H}_{1}$ of mass $M_{\mathrm{H}_{1}}=200 \mathrm{GeV}, \ldots, 800 \mathrm{GeV}$, as suggested by the names of the scenarios. The HSESM parameters of scenarios BHM200 ${ }^{ \pm}$, BHM400, and BHM600 are summarized in table 3. In the following, we take over these scenarios and refer to ref. [37] for the precise values of the SM-like input parameters.

Table 4 shows a comparison of the LO and NLO results for the decay width of the light Higgs boson, $\mathrm{H}_{2} \rightarrow 4 f$, obtained in the different renormalization schemes for each scenario. Figure 1 illustrates the scale dependence of $\Gamma^{\mathrm{H}_{2} \rightarrow 4 f}$ on the 1.h.s. for scenario BHM200+; the results for the other scenarios look qualitatively similar. The input parameters of the

\footnotetext{
${ }^{18}$ The corresponding version of PROPHECY4F can be obtained from the authors on request and will be available via www.hepforge.org soon.
} 


\begin{tabular}{|c|c|c|c|}
\hline Scenario & $M_{\mathrm{H}_{1}}[\mathrm{GeV}]$ & $s_{\alpha}$ & $\lambda_{3} / 2=\lambda_{12}^{[37]}$ \\
\hline BHM200 $^{ \pm}$ & 200 & \pm 0.29 & \pm 0.07 \\
\hline BHM400 & 400 & 0.26 & 0.17 \\
\hline BHM600 & 600 & 0.22 & 0.23 \\
\hline
\end{tabular}

Table 3. HSESM input parameters in the considered HSESM scenarios. The light Higgs boson has mass $M_{\mathrm{H}_{2}}=125.1 \mathrm{GeV}$.

\begin{tabular}{|c|c|c|c|c|}
\hline & \multicolumn{2}{|c|}{ BHM200 $^{+}$} & \multicolumn{2}{c|}{ BHM200 $^{-}$} \\
Scheme & LO & \multicolumn{1}{|c|}{ NLO } & LO & NLO \\
\hline$\overline{\mathrm{MS}(\text { PRTS })}$ & $0.83361(3)_{-4.4 \%}^{+3.2 \%}$ & $0.90539(6)_{+0.5 \%}^{+0.5 \%}$ & $0.83261(3)_{-4.5 \%}^{+3.3 \%}$ & $0.90546(7)_{+0.6 \%}^{+0.5 \%}$ \\
\hline$\overline{\mathrm{MS}}$ (FJTS) & $0.82292(3)_{+2.7 \%}^{-3.5 \%}$ & $0.90550(7)_{+0.0 \%}^{+0.7 \%}$ & $0.82614(3)_{+0.7 \%}^{-0.6 \%}$ & $0.90558(7)_{-0.1 \%}^{+0.0 \%}$ \\
\hline OS & $0.84034(3)$ & $0.90553(6)_{-0.0 \%}^{+0.0 \%}$ & $0.84034(3)$ & $0.90552(6)_{-0.0 \%}^{+0.0 \%}$ \\
\hline BFMS & $0.84036(3)$ & $0.90553(6)$ & $0.84035(3)$ & $0.90552(6)$ \\
\hline
\end{tabular}

\begin{tabular}{|c|l|l|l|l|}
\hline & \multicolumn{2}{|c|}{ BHM400 } & \multicolumn{2}{c|}{ BHM600 } \\
Scheme & \multicolumn{1}{|c|}{ LO } & \multicolumn{1}{c|}{ NLO } & LO & NLO \\
\hline$\overline{\mathrm{MS}}$ (PRTS) & $0.85209(3)_{-0.5 \%}^{+0.5 \%}$ & $0.92159(7)_{-0.0 \%}^{+0.0 \%}$ & $0.87067(3)_{-0.1 \%}^{+0.1 \%}$ & $0.94060(7)_{-0.0 \%}^{+0.0 \%}$ \\
\hline$\overline{\mathrm{MS}}$ (FJTS) & $0.85349(3)_{+1.6 \%}^{-2.1 \%}$ & $0.92166(7)_{+0.3 \%}^{+0.1 \%}$ & $0.87608(3)_{+1.2 \%}^{-1.5 \%}$ & $0.94106(7)_{+0.3 \%}^{-0.0 \%}$ \\
\hline OS & $0.85548(3)$ & $0.92178(6)_{-0.0 \%}^{+0.0 \%}$ & $0.87309(3)$ & $0.94078(7)_{-0.0 \%}^{+0.0 \%}$ \\
\hline BFMS & $0.85663(3)$ & $0.92206(6)$ & $0.87381(3)$ & $0.94118(7)$ \\
\hline
\end{tabular}

Table 4. LO and NLO decay widths $\Gamma^{\mathrm{H}_{2} \rightarrow 4 f}[\mathrm{MeV}]$ of the light HSESM Higgs boson $\mathrm{H}_{2}$ for various HSESM scenarios in different renormalization schemes, with the OS scheme as input scheme (and full conversion of the input parameters into the other schemes). The scale variation (given in percent) corresponds to the scales $\mu=\mu_{0} / 2$ and $\mu=2 \mu_{0}$ with central scale $\mu_{0}=M_{\mathrm{H}_{2}}$.

scenarios are defined in the OS scheme and consistently converted into the other schemes, as described in section 3.5 (full conversion). The residual dependence of the LO and NLO decay widths on the renormalization scale $\mu$, as obtained from a rescaling of the central scale $\mu_{0}=M_{\mathrm{H}_{2}}$ by factors of $1 / 2$ and 2 , is shown in percent as lower and upper suffixes, respectively. One crucial observation in table 4 is that all renormalization schemes deliver central NLO results differing only below the permille level, while the LO results deviate significantly by up to $2 \%$, i.e. the renormalization-scheme dependence reduces drastically in the transition from LO to NLO. Another important observation concerns the dependence on the renormalization scale. For the $\overline{\mathrm{MS}}$ (PRTS) and $\overline{\mathrm{MS}}$ (FJTS) schemes, in which the $\overline{\mathrm{MS}}$ definitions of the angle $\alpha$ lead to a running $\alpha(\mu)$, the residual scale dependence of the decay widths is reduced from $\lesssim 5 \%$ at LO to $\lesssim 0.7 \%$ at NLO. This observation was already made in ref. [37], since the $\overline{\mathrm{MS}}$ and FJ schemes from there almost coincide with the $\overline{\mathrm{MS}}$ (PRTS) and $\overline{\mathrm{MS}}$ (FJTS) schemes used here. The residual NLO scale uncertainty 

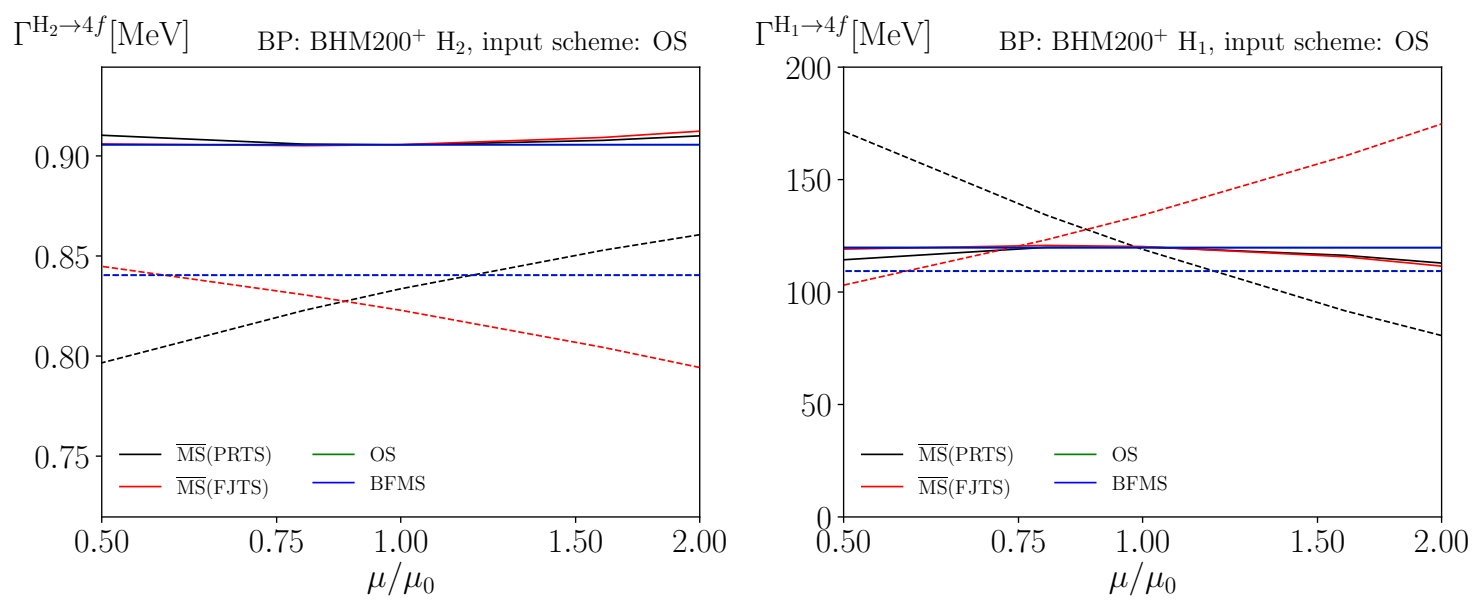

Figure 1. Scale dependence of the decay widths $\Gamma^{\mathrm{H}_{2} \rightarrow 4 f}$ (left) and $\Gamma^{\mathrm{H}_{1} \rightarrow 4 f}$ (right) of the light and heavy HSESM Higgs bosons $\mathrm{H}_{2}$ and $\mathrm{H}_{1}$ for the HSESM scenario BHM200 ${ }^{+}$in different renormalization schemes, with the OS scheme as input scheme (and full conversion of the input parameters into the other schemes). LO results are shown as dashed, NLO as full lines; the central scale is set to $\mu_{0}=M_{\mathrm{H}_{2}}$.

\begin{tabular}{|c|l|l|l|l|}
\hline & \multicolumn{2}{|c|}{ BHM200 $^{+}$} & \multicolumn{2}{c|}{ BHM200 $^{-}$} \\
Scheme & \multicolumn{1}{|c|}{ LO } & \multicolumn{1}{c|}{ NLO } & LO & NLO \\
\hline$\overline{\mathrm{MS}}$ (PRTS $)$ & $118.976(4)_{+43.6 \%}^{-31.9 \%}$ & $120.240(7)_{-4.7 \%}^{-5.9 \%}$ & $120.397(4)_{+44.6 \%}^{-32.4 \%}$ & $120.114(7)_{-5.6 \%}^{-5.7 \%}$ \\
\hline$\overline{\mathrm{MS}}$ (FJTS $)$ & $134.126(4)_{-23.2 \%}^{+30.1 \%}$ & $120.403(9)_{-1.0 \%}^{-7.1 \%}$ & $129.570(4)_{-6.0 \%}^{+5.6 \%}$ & $120.132(8)_{+0.6 \%}^{-0.1 \%}$ \\
\hline OS & $109.430(4)$ & $119.847(8)_{+0.0 \%}^{-0.0 \%}$ & $109.430(4)$ & $119.812(8)_{+0.0 \%}^{-0.0 \%}$ \\
\hline BFMS & $109.393(4)$ & $119.846(8)$ & $109.419(4)$ & $119.811(8)$ \\
\hline
\end{tabular}

\begin{tabular}{|c|l|l|l|l|}
\hline & \multicolumn{2}{|c|}{ BHM400 } & \multicolumn{2}{c|}{ BHM600 } \\
Scheme & \multicolumn{1}{|c|}{ LO } & \multicolumn{1}{c|}{ NLO } & LO & NLO \\
\hline$\overline{\mathrm{MS}}$ (PRTS $)$ & $1617.26(4)_{+6.3 \%}^{-6.2 \%}$ & $1648.62(8)_{+0.6 \%}^{-0.6 \%}$ & $4530.1(1)_{+2.1 \%}^{-2.3 \%}$ & $4546.0(2)_{+0.6 \%}^{-0.4 \%}$ \\
\hline$\overline{\mathrm{MS}}$ (FJTS $)$ & $1582.44(4)_{-21.7 \%}^{+27.6 \%}$ & $1646.83(8)_{-3.6 \%}^{-1.5 \%}$ & $4007.1(1)_{-24.8 \%}^{+32.5 \%}$ & $4509.4(3)_{-6.0 \%}^{-0.3 \%}$ \\
\hline OS & $1533.42(4)$ & $1643.86(8)_{+0.0 \%}^{-0.0 \%}$ & $4295.9(1)$ & $4532.4(2)_{+0.0 \%}^{-0.0 \%}$ \\
\hline BFMS & $1505.02(4)$ & $1636.86(9)$ & $4226.6(1)$ & $4493.8(2)$ \\
\hline
\end{tabular}

Table 5. As in table 4, but for the decay width $\Gamma^{\mathrm{H}_{1} \rightarrow 4 f}[\mathrm{MeV}]$ of the heavy HSESM Higgs boson $\mathrm{H}_{1}$. 
of $\lesssim 0.7 \%$, as estimated from rescaling $\mu_{0}$ by factors of $1 / 2$ and 2 , is, thus, of the order of magnitude typically expected from a well-behaved EW NLO calculation. Clearly, the higher-order scale dependence of the OS and BFMS schemes cannot be used as an estimate for the theoretical uncertainty, while the difference of these schemes reflects part of the renormalization-scheme dependence.

Table 5 shows a comparison of the LO and NLO results for the decay width of the heavy Higgs boson, $\mathrm{H}_{1} \rightarrow 4 f$, obtained in the different renormalization schemes for each scenario. Figure 1 illustrates the scale dependence of $\Gamma^{\mathrm{H}_{1} \rightarrow 4 f}$ on the r.h.s. for scenario BHM200 ${ }^{+}$, while again the results for the other scenarios look qualitatively similar. Note that the phenomenology of the decays $\mathrm{H}_{2} \rightarrow 4 f$ and $\mathrm{H}_{1} \rightarrow 4 f$ is rather different. Firstly, the chosen values for the mass $M_{\mathrm{H}_{1}}$ of the heavy Higgs boson are larger than $2 M_{\mathrm{W}}$ and $2 M_{\mathrm{Z}}$, so that the decays can proceed via two resonant $\mathrm{W}$ or $\mathrm{Z}$ bosons, while the decays $\mathrm{H}_{2} \rightarrow 4 f$ involve at least one off-shell $\mathrm{W} / \mathrm{Z}$ boson. This effect and the larger phase space for $\mathrm{H}_{1}$ leads to a large enhancement of the decay width for $\mathrm{H}_{1} \rightarrow 4 f$. This enhancement is somewhat damped by the second difference of the two decay types. While the LO decay width of $\mathrm{H}_{2} \rightarrow 4 f$ involves an explicit factor of $c_{\alpha}^{2}$ w.r.t. the $\mathrm{SM}$ case, the LO decay width of $\mathrm{H}_{1} \rightarrow 4 f$ contains the complementary factor $s_{\alpha}^{2}$. Since $s_{\alpha} \sim 0.2-0.3$ in the considered scenarios, $\Gamma^{\mathrm{H}_{1} \rightarrow 4 f}$ is reduced by a factor of $\sim 0.04-0.1$ w.r.t. to the $\mathrm{SM}$ decay width with the same (hypothetical) Higgs-boson mass $M_{\mathrm{H}_{1}}$. The renormalizationscale dependence in the $\overline{\mathrm{MS}}$ (PRTS) and $\overline{\mathrm{MS}}$ (FJTS) schemes is reduced by a factor $5-10$ at NLO as compared to LO. A similar reduction is observed for the differences between the renormalization schemes.

\subsubsection{Two-Higgs-doublet model}

In refs. $[29,41]$, the THDM was renormalized in four schemes, called $\overline{\operatorname{MS}}(\alpha), \mathrm{FJ}(\alpha), \overline{\operatorname{MS}}\left(\lambda_{3}\right)$, and $\operatorname{FJ}\left(\lambda_{3}\right)$ there, where the first two are identical with the $\overline{\mathrm{MS}}(\mathrm{PRTS})$ and $\overline{\mathrm{MS}}$ (FJTS) schemes of this paper, respectively. In the other two schemes, $\overline{\operatorname{MS}}\left(\lambda_{3}\right)$ and $\operatorname{FJ}\left(\lambda_{3}\right)$, the angle $\alpha$ is replaced by the scalar self-coupling $\lambda_{3}$ as $\overline{\mathrm{MS}}$-renormalized input parameter; these two schemes are not considered in the following.

In refs. [29, 41], following suggestions in the literature, various different THDM scenarios were considered, which are still compatible with current LHC results and identify the light $\mathrm{CP}$-even Higgs boson $\mathrm{H}_{2}$ with the discovered state with a mass of $125 \mathrm{GeV}$. In the following, we pick four of those scenarios, covering typical cases with light or heavy Higgs bosons in addition to the known of mass $125 \mathrm{GeV}$. Table 6 summarizes the corresponding THDM input parameters, while the remaining SM-like parameters are taken over from refs. $[29,41]$.

Table 7 shows a comparison of the LO and NLO results for the $\mathrm{H}_{2} \rightarrow 4 f$ decay width obtained in the different renormalization schemes for each scenario. Figure 2 illustrates the scale dependence of $\Gamma^{\mathrm{H}_{2} \rightarrow 4 f}$ in greater detail.

Among the two schemes with $\overline{\mathrm{MS}}$-renormalized mixing angles $\alpha$ and $\beta$, the $\overline{\mathrm{MS}}$ (PRTS) scheme delivers NLO results with a sufficiently well reduced renormalization-scale dependence of $1-2 \%$ in scenarios A1, A2, and B1, while this is the case for the $\overline{\mathrm{MS}}$ (FJTS) scheme only in scenarios A1 and B1. For scenario B2, neither the $\overline{\mathrm{MS}}$ (PRTS), nor the $\overline{\mathrm{MS}}$ (FJTS) 


\begin{tabular}{|c|c|c|c|c|c|c|}
\hline Scenario & $\begin{array}{c}M_{\mathrm{H}_{1}} \\
{[\mathrm{GeV}]}\end{array}$ & $\begin{array}{c}M_{\mathrm{H}^{+}}, M_{A_{0}} \\
{[\mathrm{GeV}]}\end{array}$ & $\lambda_{5}$ & $t_{\beta}$ & $c_{\alpha \beta}$ & comment \\
\hline A1 & 300 & 460 & -1.9 & 2 & 0.1 & Aa in refs. [29, 41] \\
\hline A2 & 300 & 460 & -1.9 & 2 & 0.2 & $\begin{array}{c}\mathrm{A}\left(c_{\alpha \beta}=0.2\right) \\
\text { in refs. }[29,41]\end{array}$ \\
\hline B1 & 600 & 690 & -1.9 & 4.5 & 0.15 & $\begin{array}{c}\text { B1 }\left(c_{\alpha \beta}=0.15\right) \\
\text { in refs. }[29,41]\end{array}$ \\
\hline B2 & 200 & 420 & -2.5746 & 3 & 0.3 & $\begin{array}{c}\text { BP3 } 3_{B 1} \text { in refs. }[29,41], \\
\text { BP3B1 in ref. [28] }\end{array}$ \\
\hline
\end{tabular}

Table 6. THDM input parameters in the considered scenarios of a THDM of Type I. The light CP-even Higgs boson has mass $M_{\mathrm{H}_{2}}=125 \mathrm{GeV}$, and $c_{\alpha \beta}=\cos (\alpha-\beta)$.

\begin{tabular}{|c|l|c|l|l|}
\hline \multirow{2}{*}{ Scheme } & \multicolumn{2}{|c|}{ L1 } & \multicolumn{2}{c|}{ A2 } \\
\hline$\overline{\mathrm{MS}}$ (PRTS) & $0.89035(3)_{+0.9 \%}^{-2.8 \%}$ & $0.96107(7)_{+0.4 \%}^{+1.2 \%}$ & $0.86130(3)_{+2.3 \%}^{-6.1 \%}$ & $0.92784(7)_{+1.3 \%}^{+1.3 \%}$ \\
\hline$\overline{\mathrm{MS}}$ (FJTS) & $0.89996(3)_{-7.4 \%}^{+0.7 \%}$ & $0.96286(7)_{-0.2 \%}^{+0.8 \%}$ & $0.88508(3)_{-10.0 \%}^{+2.2 \%}$ & $0.93605(7)_{-11.0 \%}^{+3.1 \%}$ \\
\hline OS1 & $0.89801(3)$ & $0.96218(7)_{+0.1 \%}^{-0.1 \%}$ & $0.86917(3)$ & $0.92968(7)_{+0.0 \%}^{-0.1 \%}$ \\
\hline OS2 & $0.89911(3)$ & $0.96221(7)_{+0.1 \%}^{-0.1 \%}$ & $0.87295(3)$ & $0.92995(7)_{+0.1 \%}^{-0.2 \%}$ \\
\hline OS12 & $0.89832(3)$ & $0.96197(7)_{+0.1 \%}^{-0.1 \%}$ & $0.87110(3)$ & $0.92947(7)_{+0.1 \%}^{-0.2 \%}$ \\
\hline BFMS & $0.89647(3)$ & $0.96177(7)_{+0.1 \%}^{-0.1 \%}$ & $0.86764(3)$ & $0.92914(7)_{+0.1 \%}^{-0.1 \%}$ \\
\hline
\end{tabular}

\begin{tabular}{|c|l|l|l|l|}
\hline & \multicolumn{2}{|c|}{ B1 } & \multicolumn{2}{c|}{ B2 } \\
Scheme & \multicolumn{1}{|c|}{ LO } & \multicolumn{1}{|c|}{ NLO } & LO & NLO \\
\hline$\overline{\mathrm{MS}(\mathrm{PRTS})}$ & $0.88609(3)_{+0.4 \%}^{-4.9 \%}$ & $0.94053(7)_{+1.0 \%}^{+1.1 \%}$ & $0.87941(3)_{+2.6 \%}^{-42.7 \%}$ & $0.924184(7)_{+4.2 \%}^{-39.2 \%}$ \\
\hline$\overline{\mathrm{MS}}$ (FJTS) & $0.90406(3)_{+0.4 \%}^{+0.4 \%}$ & $0.96073(7)_{-1.1 \%}^{+1.5 \%}$ & $0.90654(3)_{-2.5 \%}^{-87.0 \%}$ & $0.96980(7)_{-0.0 \%}^{++100 \%}$ \\
\hline OS1 & $0.86829(3)$ & $0.9428(1)_{-0.2 \%}^{-0.1 \%}$ & $0.82069(3)$ & $0.87179(6)_{+0.3 \%}^{-1.3 \%}$ \\
\hline OS2 & $0.88838(3)$ & $0.94136(7)_{+0.2 \%}^{-0.4 \%}$ & $0.82684(3)$ & $0.87242(6)_{+0.3 \%}^{-0.4 \%}$ \\
\hline OS12 & $0.88698(3)$ & $0.94074(7)_{+0.2 \%}^{-0.5 \%}$ & $0.82573(3)$ & $0.87189(6)_{+0.3 \%}^{-0.5 \%}$ \\
\hline BFMS & $0.88721(3)$ & $0.94113(8)_{+0.1 \%}^{-0.2 \%}$ & $0.81262(3)$ & $0.86741(6)_{+0.0 \%}^{+0.1 \%}$ \\
\hline
\end{tabular}

Table 7. LO and NLO decay widths $\Gamma^{\mathrm{H}_{2} \rightarrow 4 f}[\mathrm{MeV}]$ of the light CP-even Higgs boson $\mathrm{H}_{2}$ of the THDM for various scenarios in different renormalization schemes, with the OS12 scheme as input scheme (and full conversion of the input parameters into the other schemes). The scale variation (given in percent) corresponds to the scales $\mu=\mu_{0} / 2$ and $\mu=2 \mu_{0}$ with central scale $\mu_{0}=$ $\left(M_{\mathrm{H}_{2}}+M_{\mathrm{H}_{1}}+M_{A_{0}}+2 M_{\mathrm{H}^{+}}\right) / 5$. 

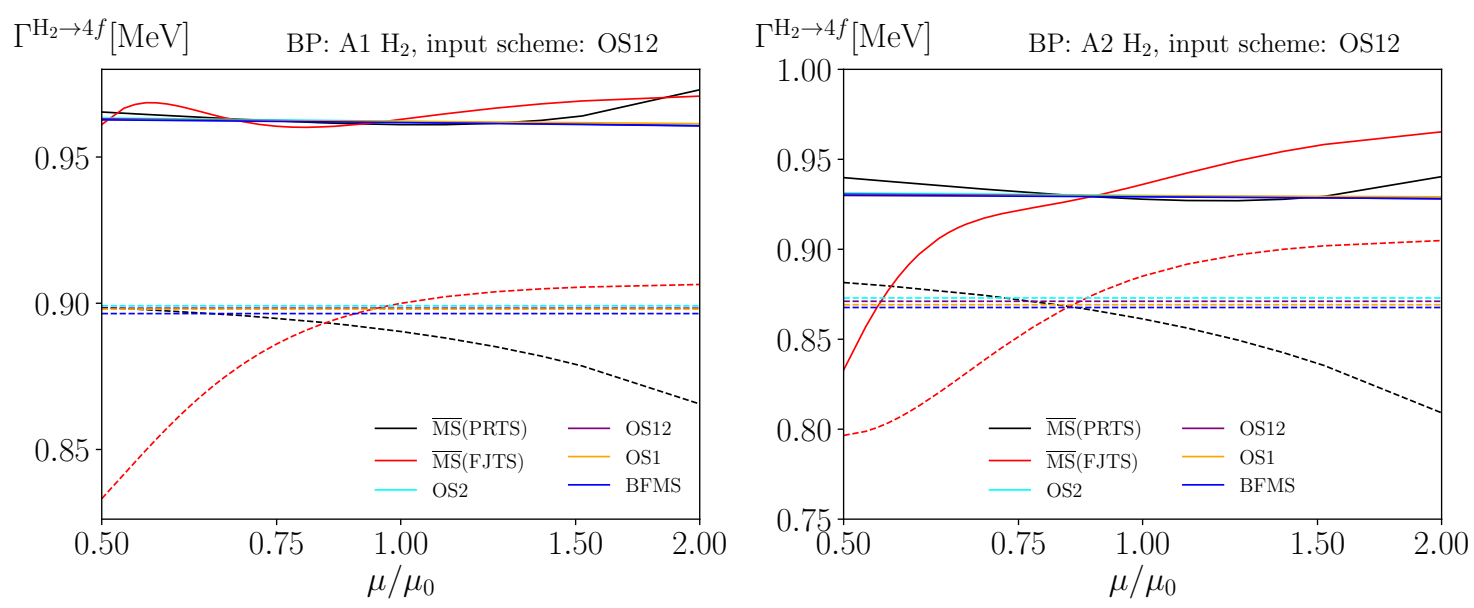

Figure 2. Scale dependence of the decay widths $\Gamma^{\mathrm{H}_{2} \rightarrow 4 f}$ of the light THDM Higgs boson $\mathrm{H}_{2}$ for the THDM scenarios A1 (left) and A2 (right) in different renormalization schemes, with the OS12 scheme as input scheme (and full conversion of the input parameters into the other schemes). LO results are shown as dashed, NLO as full lines; the central scale is set to $\mu_{0}=\left(M_{\mathrm{H}_{2}}+M_{\mathrm{H}_{1}}+M_{A_{0}}+\right.$ $\left.2 M_{\mathrm{H}^{+}}\right) / 5$.

scheme produces results with a visible stability region in $\mu$. A possible reason is the relatively small difference $M_{\mathrm{H}_{1}}-M_{\mathrm{H}_{2}}$ in the scalar masses in scenario B2 which can lead to enhanced corrections owing to the mixing renormalization constants eq. (2.5) in the $\overline{\mathrm{MS}}$ schemes. Moreover, the problems of those schemes in B2 can already be expected from the parameter conversion (cf. table 15 in appendix F) into the schemes, which involves very large corrections and shows significant differences between linearized and full conversions. It should be mentioned, however, that the versions of these $\overline{\mathrm{MS}}$-like renormalization schemes in which $\alpha$ is replaced as input parameter by the coupling parameter $\lambda_{3}$ (the schemes $\overline{\mathrm{MS}}\left(\lambda_{3}\right)$ and $\mathrm{FJ}\left(\lambda_{3}\right)$ of refs. $\left.[29,41]\right)$ behave somewhat better and at least produce some narrow plateau regions in the vicinity of the scale $\mu_{0}$ (not shown in this paper).

The OS schemes and the BFMS variant, on the other hand, deliver NLO results which agree within very few per mille, which is also the size of the residual scale dependence resulting from the running of $\lambda_{5}$ in the loop corrections. An exception is scenario B2, which shows a residual scale dependence of up to $1 \%$.

Now we turn to the discussion of the decays of the heavy CP-even Higgs boson $\mathrm{H}_{1}$. Table 8 and figure 3 show the LO and NLO results and their scale dependence for $\mathrm{H}_{1} \rightarrow 4 f$ decay width obtained in the different renormalization schemes for each scenario. Not unexpectedly, the renormalization schemes based on $\overline{\mathrm{MS}}$-renormalized mixing angles deliver problematic results. At least for the scenarios A1, A2, and B1, the NLO results of the $\overline{\mathrm{MS}}$ (PRTS) scheme still show extrema in the $\mu$ dependence that may be interpreted as plateaus of stability, but those regions are rather small and do not cover a range in $\mu$ from $\mu_{0} / 2$ to $2 \mu_{0}$. Though the extrema are located near $\mu_{0}$, and the NLO results for $\Gamma^{\mathrm{H}_{1} \rightarrow 4 f}$ in this region are compatible with the results obtained in the other stable schemes. The $\overline{\mathrm{MS}}$ (FJTS) scheme fails to produce stability regions in general, as a result of the very strong running of $\beta-\alpha$. For scenario B2, both the $\overline{\mathrm{MS}}(\mathrm{PRTS})$ and the $\overline{\mathrm{MS}}$ (FJTS) scheme fail 


\begin{tabular}{|c|l|l|l|l|}
\hline \multirow{2}{*}{ Scheme } & \multicolumn{2}{|c|}{ A1 } & \multicolumn{2}{c|}{ A2 } \\
\hline$\overline{\mathrm{MS}}$ (PRTS) & $147.102(4)_{-47.8 \%}^{>+100 \%}$ & \multicolumn{1}{|c|}{ NLO } & \multicolumn{1}{c|}{ LO } & NLO \\
\hline$\overline{\mathrm{MS}}$ (FJTS) & $64.096(2)_{>+100 \%}^{-86.9 \%}$ & $92.17(1)_{+5.6 \%}^{-81.4 \%}$ & $192.524(5)_{>+100 \%}^{-88.6 \%}$ & $318.95(5)_{>+100 \%}^{-80.2 \%}$ \\
\hline OS1 & $80.992(2)$ & $97.145(7)_{+5.1 \%}^{-5.2 \%}$ & $329.800(9)$ & $370.93(2)_{+2.7 \%}^{-2.8 \%}$ \\
\hline OS2 & $71.429(2)$ & $96.95(1)_{-0.2 \%}^{+0.1 \%}$ & $297.253(8)$ & $367.80(3)_{+0.1 \%}^{-0.3 \%}$ \\
\hline OS12 & $78.304(2)$ & $98.812(8)_{+0.7 \%}^{-0.8 \%}$ & $313.217(8)$ & $371.86(3)_{+0.6 \%}^{-0.7 \%}$ \\
\hline BFMS & $94.265(2)$ & $100.117(5)_{+1.6 \%}^{-2.2 \%}$ & $343.049(9)$ & $375.22(2)_{+1.3 \%}^{-1.7 \%}$ \\
\hline
\end{tabular}

\begin{tabular}{|c|c|c|c|c|}
\hline \multirow[b]{2}{*}{ Scheme } & \multicolumn{2}{|c|}{ B1 } & \multicolumn{2}{|c|}{$\mathrm{B} 2$} \\
\hline & $\mathrm{LO}$ & NLO & $\mathrm{LO}$ & NLO \\
\hline$\overline{\mathrm{MS}}(\mathrm{PRTS})$ & $2083.68(5)_{-17.9 \%}^{>+100 \%}$ & $2162.3(1)_{-27.0 \%}^{<-100 \%}$ & $40.122(1)_{-82.7 \%}^{>+100 \%}$ & $57.73(1)_{-82.7 \%}^{>+100 \%}$ \\
\hline$\overline{\mathrm{MS}}(\mathrm{FJTS})$ & $325.923(7)_{-100 \%}^{-99.6 \%}$ & $1179.8(3)_{-99.8 \%}^{-99.5 \%}$ & $1.21460(4)_{>+100 \%}^{>+100 \%}$ & $\begin{aligned}-5.226(3)_{>+100 \%}^{<-100 \%} \\
\end{aligned}$ \\
\hline OS1 & $3824.52(9)$ & $\begin{array}{l}1542.9(9)_{+}^{-21.5 \%} \\
+28.2 \% \\
\end{array}$ & $124.325(4)$ & $132.307(8)_{+1.9 \%}^{+4.6 \%}$ \\
\hline OS2 & $1860.03(4)$ & $2130.3(1)_{+0.1 \%}^{-1.6 \%}$ & $115.515(4)$ & $131.09(1)_{+2.4 \%}^{-4.2 \%}$ \\
\hline OS12 & $1997.07(5)$ & $2155.2(1)_{+0.7 \%}^{-1.4 \%}$ & $117.108(4)$ & $131.88(1)_{+2.2 \%}^{-2.9 \%}$ \\
\hline BFMS & $1973.68(5)$ & $2145.0(1)_{+3.6 \%}^{-9.5 \%}$ & $135.904(5)$ & $138.729(8)_{+4.7 \%}^{-7.7 \%}$ \\
\hline
\end{tabular}

Table 8. As in table 7, but for the decay width $\Gamma^{\mathrm{H}_{1} \rightarrow 4 f}[\mathrm{MeV}]$ of the heavy CP-even Higgs boson $\mathrm{H}_{1}$ of the THDM.
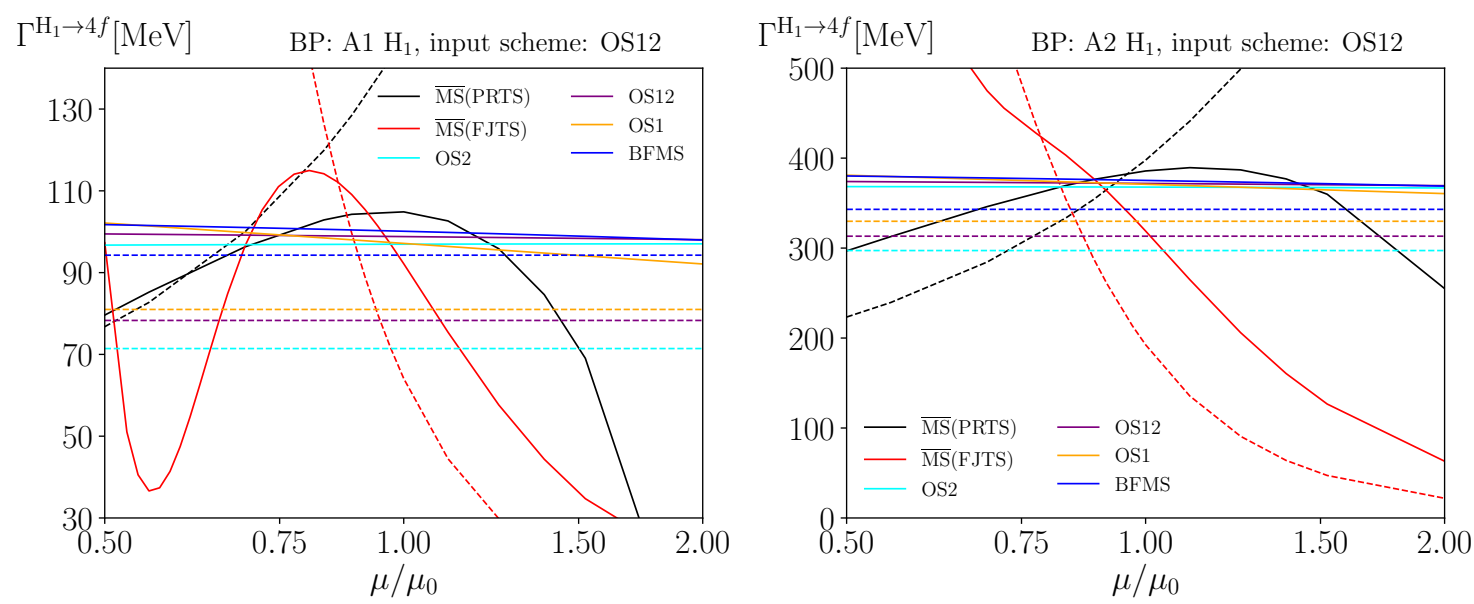

Figure 3. As in figure 2, but for the decay width $\Gamma^{\mathrm{H}_{1} \rightarrow 4 f}$ of the heavy CP-even Higgs boson of the THDM. 
badly, as expected already from the bad behaviour observed for the decay of the light Higgs boson $\mathrm{H}_{2}$ above. The OS and BFMS schemes, however, mostly deliver NLO results that are in nice mutual agreement, with the only exception of the OS1 scheme in scenario B1, where the running of $\lambda_{5}$ in the loop corrections introduces a scale uncertainty of the order of $20-30 \%$. Note that also the NLO correction in this scheme is extremely large.

\subsection{Higgs-boson production processes at the LHC}

As a second application of our renormalization schemes, we consider the EW corrections to the production of BSM Higgs bosons in association with a vector boson, also known as Higgs-strahlung, and in association with two jets, usually referred to as vector-boson fusion (VBF). For the numerical integration we have used a modified version of the Monte Carlo program HAwK 2.0 [59]. HAWK 2.0 is a Monte Carlo integrator for Higgs-strahlung and VBF in the SM, including the full fixed-order NLO QCD and EW corrections [61-63]. The modification of HAWK 2.0 concerns an interface with the RECOLA2 library which has been introduced in ref. [27]. RECOLA2, the successor of RECOLA [64], is a highly efficient one-loop amplitude provider for the SM and BSM theories which is publicly available [65]. All necessary ingredients for the integration of the before-mentioned processes for BSM theories can be automatically generated by RECOLA 2 which, in turn, upgrades the original HAWK 2.0 to a general integrator for BSM Higgs production in Higgs-strahlung and VBF as long as no external charged Higgs boson is considered. A new release of HAwk 2.0 will be made available shortly.

\subsubsection{Cut setup and parameters}

For the analysis of Higgs-strahlung we focus on the final state with one charged muon and a muon-neutrino, $\mathrm{pp} \rightarrow \mathrm{H} \mu^{+} \nu_{\mu}+X$ at $13 \mathrm{TeV}$. The muon is not recombined with collinear photons, and is assumed to be perfectly isolated,treated as bare muon as described in ref. [63]. We use similar cuts to the ones given in [66], i.e. we demand the muon

- has transverse momentum $p_{\mathrm{T}, \mu^{+}}>20 \mathrm{GeV}$,

- be central with rapidity $\left|y_{\mu^{+}}\right|<2.4$,

and require a missing transverse momentum of $p_{\mathrm{T}}^{\text {miss }}>25 \mathrm{GeV}$.

For the Higgs-boson production in VBF we require two hards jets emitted from partons $i$ which fulfil

- pseudo-rapidity $\left|\eta_{i}\right|<5$.

The jet definition is performed using the anti- $k_{\mathrm{T}}$ algorithm [67] with jet size $D=0.4$. The jets $\mathrm{j}_{i}, i=1,2$, are required to fulfil typical VBF cuts (see e.g. ref. [68]):

- transverse momentum $p_{\mathrm{T}, \mathrm{j}_{i}}>20 \mathrm{GeV}$,

- rapidity $\left|y_{\mathrm{j}_{i}}\right|<5$,

- rapidity difference $\left|y_{\mathrm{j}_{1}}-y_{\mathrm{j}_{2}}\right|>3$,

- opposite hemispheres $y_{\mathrm{j}_{1}} y_{\mathrm{j}_{2}}<0$, 


\begin{tabular}{|c|c|c|l|c|}
\hline & \multicolumn{2}{|c|}{ BHM200 $^{+}$} & \multicolumn{2}{c|}{ BHM200 $^{-}$} \\
Scheme & LO & \multicolumn{1}{|c|}{ NLO } & LO & NLO \\
\hline$\overline{\mathrm{MS}}$ (PRTS) & $42.61(2)_{-4.5 \%}^{+3.2 \%}$ & $40.80(2)_{+1.2 \%}^{+0.1 \%}$ & $42.55(2)_{-4.6 \%}^{+3.3 \%}$ & $40.80(2)_{+1.3 \%}^{+0.1 \%}$ \\
\hline$\overline{\mathrm{MS}}$ (FJTS) & $42.12(2)_{+2.7 \%}^{-3.5 \%}$ & $40.90(2)_{-0.3 \%}^{+1.3 \%}$ & $42.23(2)_{+0.7 \%}^{-0.6 \%}$ & $40.85(2)_{-0.2 \%}^{+0.1 \%}$ \\
\hline OS & $42.95(2)$ & $40.74(2)_{+0.0 \%}^{+0.0 \%}$ & $42.96(2)$ & $40.74(2)_{-0.0 \%}^{-0.0 \%}$ \\
\hline BFMS & $42.95(2)$ & $40.74(2)$ & $42.96(2)$ & $40.74(2)$ \\
\hline
\end{tabular}

\begin{tabular}{|c|l|l|l|l|}
\hline & \multicolumn{2}{|c|}{ BHM400 } & \multicolumn{2}{c|}{ BHM600 } \\
Scheme & LO & \multicolumn{1}{c|}{ NLO } & \multicolumn{1}{c|}{ LO } & NLO \\
\hline$\overline{\mathrm{MS}}$ (PRTS) & $43.56(2)_{-0.5 \%}^{+0.5 \%}$ & $41.49(2)_{+0.0 \%}^{-0.0 \%}$ & $44.51(2)_{-0.1 \%}^{+0.1 \%}$ & $42.33(3)_{+0.0 \%}^{+0.0 \%}$ \\
\hline$\overline{\mathrm{MS}}$ (FJTS) & $43.63(2)_{+1.7 \%}^{-2.1 \%}$ & $41.48(2)_{+0.1 \%}^{+0.4 \%}$ & $44.79(2)_{+1.2 \%}^{-1.6 \%}$ & $42.33(3)_{+0.2 \%}^{+0.2 \%}$ \\
\hline OS & $43.73(2)$ & $41.42(2)_{+0.0 \%}^{+0.0 \%}$ & $44.63(2)$ & $42.31(3)_{-0.0 \%}^{+0.0 \%}$ \\
\hline BFMS & $43.78(2)$ & $41.48(2)$ & $44.63(2)$ & $42.31(3)$ \\
\hline
\end{tabular}

Table 9. LO and NLO integrated cross sections $\sigma$ [pb] for the production of the light Higgs boson in Higgs-strahlung, $\mathrm{pp} \rightarrow \mathrm{H}_{2} \mu^{+} \nu_{\mu}+X$, for various HSESM scenarios in different renormalization schemes, with the OS scheme as input scheme (and full conversion of the input parameters into the other schemes). The scale variation (given in percent) corresponds to the scales $\mu=\mu_{0} / 2$ and $\mu=2 \mu_{0}$ with central scale $\mu_{0}=M_{\mathrm{H}_{2}}$.

- invariant mass $M_{\mathrm{j}_{1} \mathrm{j}_{2}}>130 \mathrm{GeV}$.

For Higgs-strahlung we investigate all the benchmark scenarios given in table 3 and table 6 in the HSESM and THDM, respectively. For VBF we only consider the points A1 and A2 in table 6 in the THDM, since in general the results look pretty similar to those for Higgs-strahlung.

\subsubsection{Higgs-strahlung in the HSESM}

In tables 9 and 10 we show the results for pp $\rightarrow \mathrm{H} \mu^{+} \nu_{\mu}+X$ for $\mathrm{H}=\mathrm{H}_{2}$ and $\mathrm{H}_{1}$, respectively. For the light-Higgs-boson production in table 9 the LO scale dependence is small, ranging from $\sim 1.5 \%$ for BHM400 and BHM600 to $\sim 3 \%$ for BHM $200^{ \pm}$. The scale uncertainty gets significantly reduced at NLO by a factor of $4-5$. The NLO scale uncertainty for all on-shell schemes and the $\overline{\mathrm{MS}}$ (PRTS) are below 0.05\%. Overall, the observed EW corrections are small, and central results are consistent within all schemes, with a scheme dependence at the permille level. A detailed scale dependence of BHM200 ${ }^{+}$and BHM200- ${ }^{-}$is shown in figure 4.

For heavy-Higgs-boson production $\mathrm{H}_{1}$ in table 10 the picture is qualitatively the same with the difference being that the size of the corrections and the scale uncertainties are amplified with respect to light-Higgs-boson production. However, phenomenologically, the scenario corresponds to an entirely different situation, since the heavy Higgs boson is only 

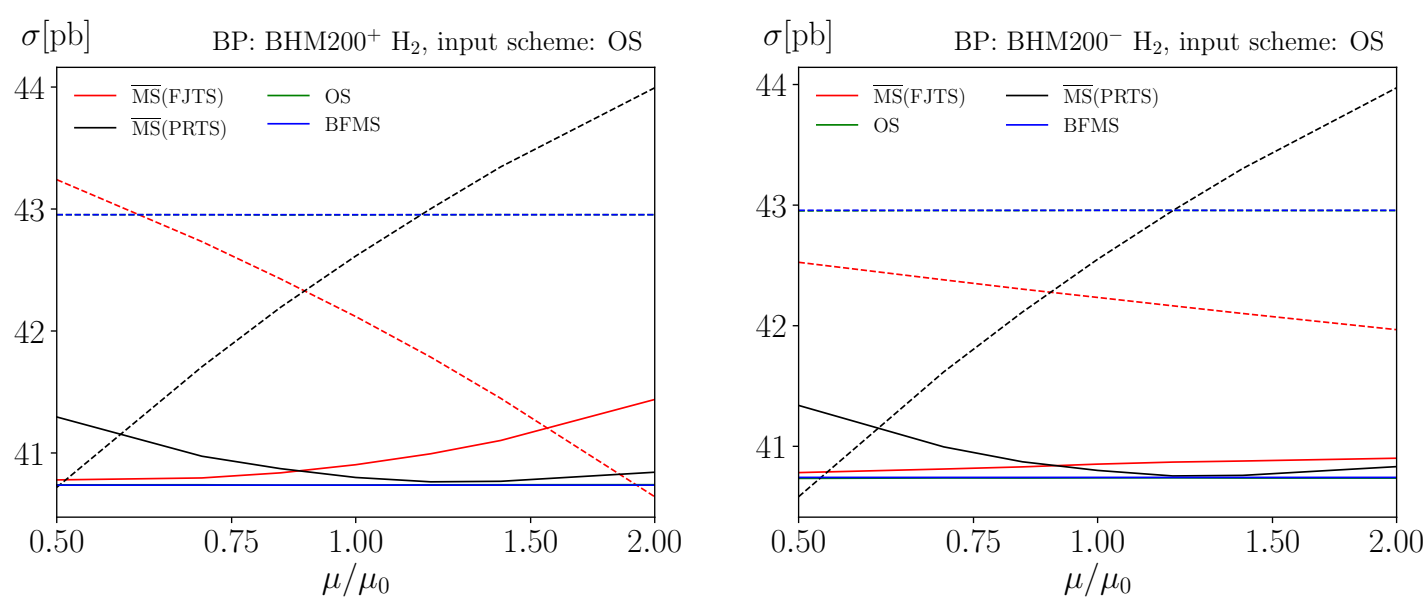

Figure 4. Scale dependence for light-Higgs-boson production in Higgs-strahlung, pp $\rightarrow \mathrm{H}_{2} \mu^{+} \nu_{\mu}+$ $X$, for the HSESM benchmark scenarios BHM200+ (left) and BHM200- (right) in different renormalization schemes, with the OS scheme as input scheme (and full conversion of the input parameters into the other schemes). LO results are shown as dashed, NLO as full lines; the central scale is set to $\mu_{0}=M_{\mathrm{H}_{2}}$.
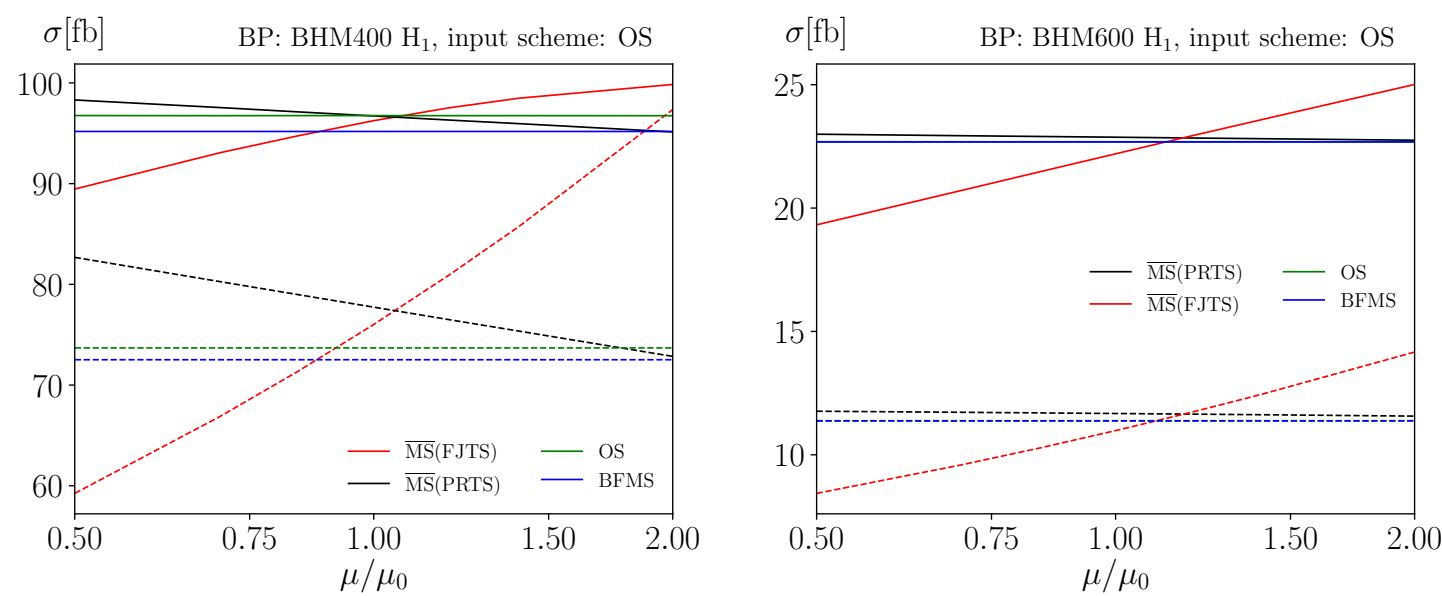

Figure 5. As in figure 4, but for $\mathrm{H}_{1}$ production in the benchmark scenarios BHM400 (left) and BHM600 (right).

coupling weakly to the vector bosons and loop contributions significantly contribute to the production, resulting in large EW corrections. In Figure 5 we depict the scale dependence for BHM400 and BHM600. While for BHM400 the $\overline{\mathrm{MS}}$ schemes show a significant reduction in the scale uncertainty, for BHM600 the absolute scale variation does not change from LO to NLO, though a reduction of the relative scale uncertainty results from the increase of the integrated cross section. It is notable that even though the corrections reach up to $100 \%$ all schemes agree at the central scale within less than about $2 \%$. We conclude that also for the heavy-Higgs-boson production all schemes are mutually consistent with no sign of artificially enhanced corrections. 


\begin{tabular}{|c|l|l|l|l|}
\hline \multirow{2}{*}{ Scheme } & \multicolumn{2}{|c|}{ BHM200 $^{+}$} & \multicolumn{2}{c|}{ BHM200- $^{-}$} \\
\hline$\overline{\mathrm{MS}}$ (PRTS) & $1039.9(4)_{+44.4 \%}^{-32.3 \%}$ & $915(1)_{-11.8 \%}^{-2.3 \%}$ & $1055.4(4)_{+45.3 \%}^{-32.8 \%}$ & $918(1)_{-12.6 \%}^{-2.1 \%}$ \\
\hline$\overline{\mathrm{MS}}$ (FJTS) & $1161.3(5)_{-23.6 \%}^{+30.8 \%}$ & $893(2)_{+2.6 \%}^{-13.3 \%}$ & $1132.5(4)_{-6.2 \%}^{+5.8 \%}$ & $908(2)_{+1.6 \%}^{-1.1 \%}$ \\
\hline OS & $957.8(4)$ & $926.6(7)_{+0.0 \%}^{-0.0 \%}$ & $957.9(4)$ & $928.8(7)_{+0.0 \%}^{+0.0 \%}$ \\
\hline BFMS & $957.5(4)$ & $926.3(7)$ & $957.7(4)$ & $928.7(7)$ \\
\hline
\end{tabular}

\begin{tabular}{|c|l|l|l|l|}
\hline & \multicolumn{2}{|c|}{ BHM400 } & \multicolumn{2}{c|}{ BHM600 } \\
Scheme & \multicolumn{1}{|c|}{ LO } & \multicolumn{1}{c|}{ NLO } & LO & NLO \\
\hline$\overline{\mathrm{MS}}$ (PRTS) & $77.73(3)_{+6.4 \%}^{-6.3 \%}$ & $96.72(9)_{+1.6 \%}^{-1.6 \%}$ & $11.992(4)_{+2.1 \%}^{-2.3 \%}$ & $23.20(1)_{+1.3 \%}^{-1.3 \%}$ \\
\hline$\overline{\mathrm{MS}}$ (FJTS) & $76.01(3)_{-2.1 \%}^{+28.1 \%}$ & $96.25(8)_{-7.1 \%}^{+3.7 \%}$ & $10.586(3)_{-25.2 \%}^{+33.1 \%}$ & $22.769(9)_{-14.8 \%}^{+14.8 \%}$ \\
\hline OS & $72.69(2)$ & $96.75(6)_{+0.0 \%}^{-0.0 \%}$ & $11.380(3)$ & $22.69(1)_{+0.0 \%}^{-0.0 \%}$ \\
\hline BFMS & $72.52(2)$ & $95.18(6)$ & $11.376(3)$ & $22.71(1)$ \\
\hline
\end{tabular}

Table 10. As in table 9, but for the cross section $\sigma$ [fb] of heavy Higgs-boson production in Higgs-strahlung, $\mathrm{pp} \rightarrow \mathrm{H}_{1} \mu^{+} \nu_{\mu}+X$, in the HSESM.

\subsubsection{Higgs-strahlung in the THDM}

The results for Higgs production in Higgs-strahlung in the THDM are shown in tables 11 and 12 for the light $\left(\mathrm{H}_{2}\right)$ and heavy $\left(\mathrm{H}_{1}\right)$ Higgs-boson production, respectively, with the OS12 as input scheme. Figures 6 and 7 show the scale dependence for $\mathrm{pp} \rightarrow \mathrm{H}_{1,2} \mu^{+} \nu_{\mu}+X$ for different renormalization schemes.

As illustrated in figure 6, the on-shell schemes yield stable results for light-Higgs-boson production. Table 11 shows that neglecting B2 for the moment and comparing to Higgsstrahlung in the HSESM, the reduction of the scale uncertainty is either less strong (e.g. for A1, A2, B1 in $\overline{\mathrm{MS}}$ (PRTS), and A1 in $\overline{\mathrm{MS}}$ (FJTS)) or not observed (A2, B1 in $\overline{\mathrm{MS}}$ (FJTS)). Nonetheless, the results for the benchmark scenarios A1, A2, and B1 visibly agree in all schemes within $2 \%$ at NLO, apart from the $\overline{\mathrm{MS}}$ (FJTS) scheme for B1. For B2 the $\overline{\mathrm{MS}}$ schemes show large scale uncertainties, and no stabilization of the results is visible when going from LO to NLO. The central values for the $\overline{\mathrm{MS}}$ schemes differ by up to $9 \%$ from the results for the on-shell schemes, while the latter mutually agree at the permille level.

As can be seen in table 12, for the heavy-Higgs-boson production in general the scale uncertainty within the traditional $\left[\mu_{0} / 2,2 \mu_{0}\right]$ window remains large within the $\overline{\mathrm{MS}}$ schemes. Nonetheless, extrema or at least regions of smaller scale dependence show up for the benchmark scenarios A1 and A2 (cf. figure 7) which can be viewed as narrow plateaus.

Focusing on scenario A1 the scale uncertainty is still large at NLO in the $\overline{\mathrm{MS}}$ schemes, while the results in the on-shell schemes are well consistent and their spread decreases at NLO. Yet uncertainties of $4 \%$ are visible for OS1 which are not unexpected due to the genuinely large corrections for heavy-Higgs-boson production in all on-shell schemes. The 

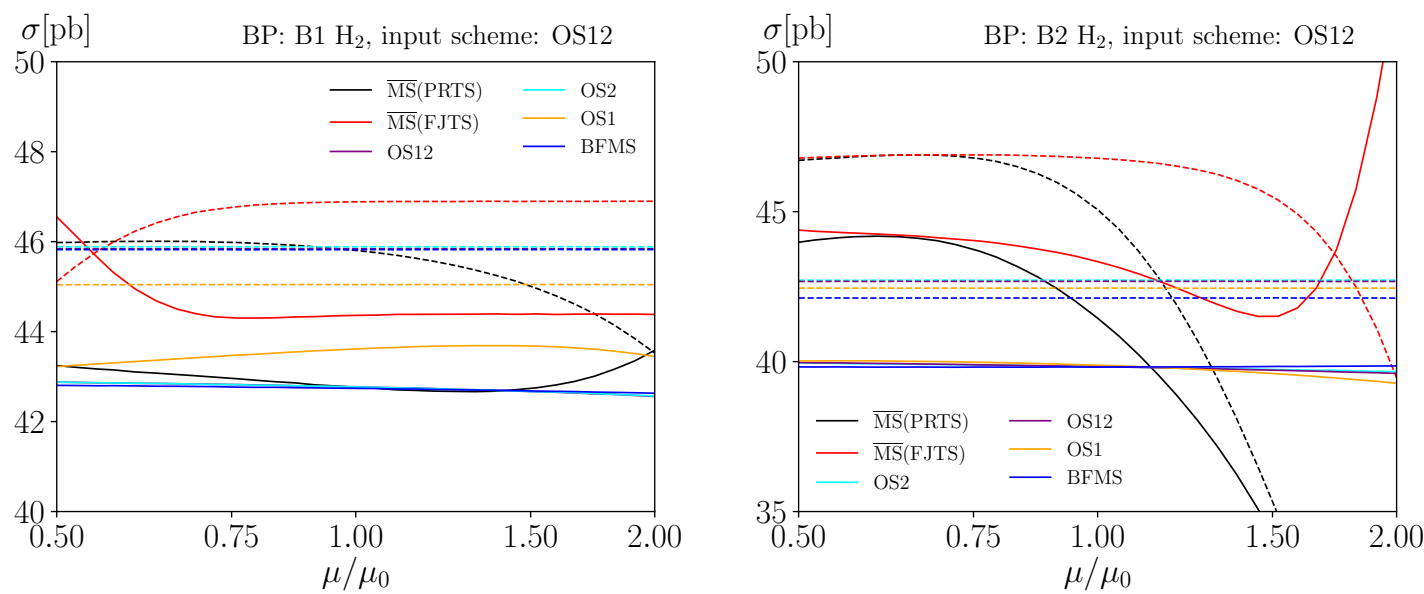

Figure 6. Scale dependence for light-Higgs-boson production in Higgs-strahlung, pp $\rightarrow \mathrm{H}_{2} \mu^{+} \nu_{\mu}+$ $X$, for the THDM benchmark scenarios B1 (left) and B2 (right) in different renormalization schemes, with the OS12 scheme as input scheme (and full conversion of the input parameters into the other schemes). LO results are shown as dashed, NLO as full lines; the central scale is set to $\mu_{0}=$ $\left(M_{\mathrm{H}_{2}}+M_{\mathrm{H}_{1}}+M_{A_{0}}+2 M_{\mathrm{H}^{+}}\right) / 5$.
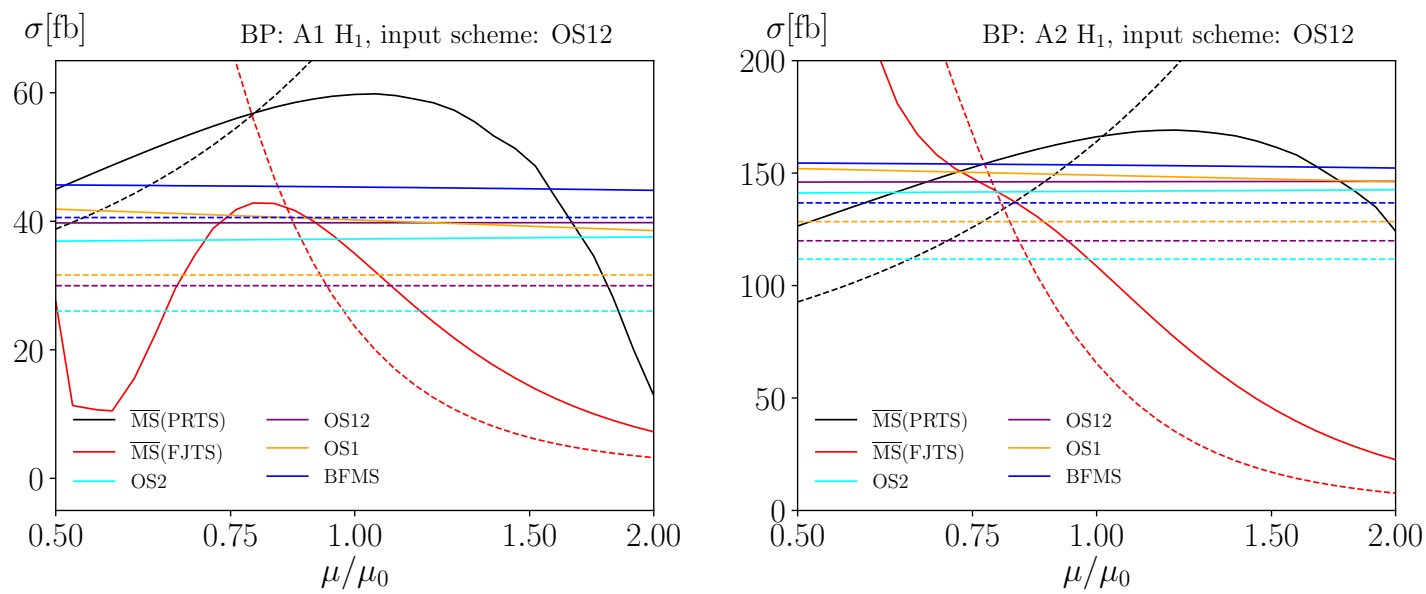

Figure 7. Scale dependence for heavy-Higgs-boson production in Higgs-strahlung, pp $\rightarrow \mathrm{H}_{1} \mu^{+} \nu_{\mu}+$ $X$, for the THDM benchmark scenarios A1 (left) and A2 (right) in different renormalization schemes, with the OS12 scheme as input scheme (and full conversion of the input parameters into the other schemes). LO results are shown as dashed, NLO as full lines; the central scale is set to $\mu_{0}=$ $\left(M_{\mathrm{H}_{2}}+M_{\mathrm{H}_{1}}+M_{A_{0}}+2 M_{\mathrm{H}^{+}}\right) / 5$. 


\begin{tabular}{|c|l|c|l|c|}
\hline & \multicolumn{2}{|c|}{ A1 } & \multicolumn{2}{c|}{ A2 } \\
Scheme & LO & NLO & LO & NLO \\
\hline$\overline{\mathrm{MS}(\text { PRTS })}$ & $45.76(2)_{+1.2 \%}^{-3.4 \%}$ & $43.67(2)_{+0.4 \%}^{+2.1 \%}$ & $44.33(2)_{+2.5 \%}^{-6.4 \%}$ & $42.25(2)_{+1.2 \%}^{+2.5 \%}$ \\
\hline$\overline{\mathrm{MS}}$ (FJTS) & $46.53(2)_{-7.2 \%}^{+0.7 \%}$ & $43.93(3)_{+1.4 \%}^{+0.7 \%}$ & $45.87(2)_{-10.2 \%}^{+2.0 \%}$ & $42.90(3)_{-9.2 \%}^{+2.6 \%}$ \\
\hline OS1 & $46.40(2)$ & $43.88(3)_{+0.0 \%}^{-0.1 \%}$ & $44.88(2)$ & $42.43(3)_{+0.0 \%}^{-0.0 \%}$ \\
\hline OS2 & $46.49(2)$ & $43.90(3)_{+0.1 \%}^{-0.1 \%}$ & $45.15(2)$ & $42.49(3)_{+0.1 \%}^{-0.2 \%}$ \\
\hline OS12 & $46.43(2)$ & $43.87(3)_{+0.1 \%}^{-0.1 \%}$ & $45.02(2)$ & $42.44(3)_{+0.1 \%}^{-0.2 \%}$ \\
\hline BFMS & $46.26(2)$ & $46.82(3)_{+0.1 \%}^{-0.1 \%}$ & $46.76(2)$ & $46.38(3)_{+0.1 \%}^{-0.1 \%}$ \\
\hline
\end{tabular}

\begin{tabular}{|c|l|c|l|c|}
\hline & \multicolumn{2}{|c|}{ B1 } & \multicolumn{2}{c|}{ B2 } \\
Scheme & LO & NLO & LO & NLO \\
\hline$\overline{\mathrm{MS}(\mathrm{PRTS})}$ & $45.81(2)_{+0.4 \%}^{-5.0 \%}$ & $42.76(3)_{+1.1 \%}^{+1.9 \%}$ & $45.07(2)_{+3.6 \%}^{-44.5 \%}$ & $41.45(3)_{+6.1 \%}^{-42.1 \%}$ \\
\hline$\overline{\mathrm{MS}}$ (FJTS) & $46.89(2)_{-3.8 \%}^{+0.0 \%}$ & $44.36(3)_{+4.9 \%}^{+0.0 \%}$ & $46.78(2)_{+0.0 \%}^{-15.6 \%}$ & $43.33(3)_{+2.4 \%}^{+21.4 \%}$ \\
\hline OS1 & $45.04(2)$ & $43.61(2)_{-0.9 \%}^{-0.4 \%}$ & $42.45(2)$ & $39.88(3)_{+0.3 \%}^{-1.5 \%}$ \\
\hline OS2 & $45.88(2)$ & $42.77(3)_{+0.3 \%}^{-0.5 \%}$ & $42.72(2)$ & $39.83(3)_{+0.3 \%}^{-0.4 \%}$ \\
\hline OS12 & $45.84(2)$ & $42.77(3)_{+0.2 \%}^{-0.5 \%}$ & $42.68(2)$ & $39.83(3)_{+0.3 \%}^{-0.6 \%}$ \\
\hline BFMS & $45.82(2)$ & $42.74(3)_{+0.2 \%}^{-0.3 \%}$ & $42.12(2)$ & $39.82(2)_{+0.0 \%}^{+0.1 \%}$ \\
\hline
\end{tabular}

Table 11. LO and NLO integrated cross sections $\sigma[\mathrm{pb}]$ for the production of the light Higgs boson in Higgs-strahlung, pp $\rightarrow \mathrm{H}_{2} \mu^{+} \nu_{\mu}+X$, for various THDM scenarios in different renormalization schemes, with the OS12 scheme as input scheme (and full conversion of the input parameters into the other schemes). The scale variation (given in percent) corresponds to the scales $\mu=\mu_{0} / 2$ and $\mu=2 \mu_{0}$ with central scale $\mu_{0}=\left(M_{\mathrm{H}_{2}}+M_{\mathrm{H}_{1}}+M_{A_{0}}+2 M_{\mathrm{H}^{+}}\right) / 5$.

large corrections are due to the proximity of the scenario A1 to the alignment limit and also cause large conversion effects between the on-shell or BFM and $\overline{\mathrm{MS}}$ schemes (see table 15 in appendix F). For scenario A2, which is further away from the alignment limit, the picture is slightly better.

For scenario B1 the conversion effects between the schemes $\overline{\mathrm{MS}}$ (PRTS), OS2, OS12, and BFMS are small (see table 15). This is reflected in a LO prediction of similar size and a surprisingly good agreement at NLO between those schemes, even though the corrections exceed $100 \%$ in heavy-Higgs-boson production. The $\overline{\mathrm{MS}}$ (FJTS) scheme fails to give any reasonable result in heavy-Higgs-boson production due to the large conversion effects pushing into the alignment limit $c_{\alpha \beta} \approx 0$. The large conversion effects observed for the OS1 scheme can be traced back to the renormalization of $\beta$, and replacing (3.21) with e.g. (3.24), but keeping the renormalization of $\alpha$ fixed, results in small conversion effects. While, we could not completely disentangle the reason for the large effects in (3.21), this is a least partly caused by the enhancement proportional to $t_{\beta}$ in (3.21) which is not present in the OS2 (3.24), OS12 (3.30), and BFMS schemes. While the OS2 scheme might still 


\begin{tabular}{|c|c|c|c|c|}
\hline \multirow[b]{2}{*}{ Scheme } & \multicolumn{2}{|c|}{$\mathrm{A} 1$} & \multicolumn{2}{|c|}{$\mathrm{A} 2$} \\
\hline & $\mathrm{LO}$ & NLO & $\mathrm{LO}$ & NLO \\
\hline$\overline{\mathrm{MS}}$ (PRTS) & $72.47(3)_{-46.5 \%}^{>+100 \%}$ & $59.7(2)_{-24.7 \%}^{-79.4 \%}$ & $164.02(6)_{-43.5 \%}^{>+100 \%}$ & $166.2(2)_{-23.9 \%}^{-25.4 \%}$ \\
\hline$\overline{\mathrm{MS}}$ (FJTS) & $23.647(9)_{>+100 \%}^{-86.4 \%}$ & $34.98(3)_{-27.0 \%}^{-79.3 \%}$ & $65.42(2)_{>+100 \%}^{-88.3 \%}$ & $108.6(1)_{>+100 \%}^{-79.2 \%}$ \\
\hline OS1 & $31.64(1)$ & $40.21(2)_{+4.2 \%}^{-4.1 \%}$ & $128.41(5)$ & $149.10(8)_{+2.0 \%}^{-2.0 \%}$ \\
\hline OS2 & $26.03(1)$ & $37.25(3)_{-0.9 \%}^{+0.8 \%}$ & $111.73(4)$ & $141.89(5)_{-0.5 \%}^{+0.5 \%}$ \\
\hline OS12 & $29.98(1)$ & $39.78(2)_{-0.1 \%}^{+0.0 \%}$ & $119.92(4)$ & $146.18(5)_{-0.1 \%}^{+0.1 \%}$ \\
\hline BFMS & $40.58(1)$ & $\begin{array}{l}45.33(4)_{+0.7 \%}^{-1.1 \%} \\
\end{array}$ & $136.78(5)$ & $153.6(1)_{+0.6 \%}^{-0.9 \%}$ \\
\hline
\end{tabular}

\begin{tabular}{|c|l|l|l|l|}
\hline \multirow{2}{*}{ Scheme } & \multicolumn{2}{|c|}{ B1 } & \multicolumn{2}{c|}{ B2 } \\
\hline$\overline{\mathrm{MS}}$ (PRTS) & $5.458(2)_{-15.5 \%}^{>+100 \%}$ & \multicolumn{1}{|c|}{ NLO } & \multicolumn{1}{c|}{ LO } & \multicolumn{1}{c|}{ NLO } \\
\hline$\overline{\mathrm{MS}}$ (FJTS) & $0.05296(2)_{>++100 \%}^{-99.8 \%}$ & $0.1243(1)_{--100 \%}^{-96.9 \%}$ & $27.85(1)_{-1.3 \%}^{++100 \%}$ & $161.7(7)_{<-100 \%}^{<-100 \%}$ \\
\hline OS1 & $9.316(5)$ & $9.20(6)_{+30.2 \%}^{-7.7 \%}$ & $1079.8(4)$ & $1015.6(9)_{+1.4 \%}^{+6.7 \%}$ \\
\hline OS2 & $5.076(2)$ & $10.954(4)_{-0.4 \%}^{-0.1 \%}$ & $1015.7(4)$ & $1022.6(5)_{+2.1 \%}^{-3.9 \%}$ \\
\hline OS12 & $5.291(2)$ & $11.051(4)_{-0.0 \%}^{+0.0 \%}$ & $1025.1(4)$ & $1023.6(5)_{+1.8 \%}^{-2.5 \%}$ \\
\hline BFMS & $5.386(2)$ & $11.303(4)_{+1.5 \%}^{-4.4 \%}$ & $1159.8(5)$ & $1032(1)_{++4.9 \%}^{-8.4 \%}$ \\
\hline
\end{tabular}

Table 12. As in table 11, but for the cross section $\sigma$ [fb] of heavy Higgs-boson production in Higgs-strahlung, $\mathrm{pp} \rightarrow \mathrm{H}_{1} \mu^{+} \nu_{\mu}+X$, in the THDM.

be affected by similar problems in certain parameter regions, the scheme OS12 is free of such artificial enhancements and therefore preferable. The seemingly fair agreement between the OS1 and the other on-shell and BFM schemes at NLO is just accidental, since for the Higgs decay (table 8) no good agreement is found. We conclude that the schemes OS2, OS12, BFMS, and $\overline{\mathrm{MS}}$ (PRTS) give consistent predictions even though the $K$ factor is about 2 .

For scenario B2, conversion effects between $\overline{\mathrm{MS}}$ and on-shell or BFM schemes (see table 15) are sizeable, while within the on-shell and BFM schemes these are small. For the on-shell and BFM schemes, even for heavy-Higgs-boson production the corrections are well behaved, ranging between $-11 \%$ and $+1 \%$. The $\overline{\mathrm{MS}}$ schemes suffer from very large scale uncertainties and large corrections at the central scale.

\subsubsection{Higgs production via vector-boson fusion in the THDM}

For Higgs production via VBF we provide only some exemplary results. We show the scale dependence for light-Higgs-boson production in A1 and A2 in figure 8, and summarize the usual scale variation in table 13. Since the behaviour of these results resembles closely the one for Higgs-strahlung, apart from the magnitude of the cross sections, we did not investi- 


\begin{tabular}{|c|l|c|l|c|}
\hline & \multicolumn{2}{|c|}{ A1 } & \multicolumn{2}{c|}{ A2 } \\
Scheme & \multicolumn{1}{|c|}{ LO } & NLO & LO & NLO \\
\hline$\overline{\mathrm{MS}}$ (PRTS) & $2145.4(6)_{+1.1 \%}^{-3.4 \%}$ & $2029.7(8)_{+0.5 \%}^{+2.0 \%}$ & $2078.3(6)_{+2.5 \%}^{-6.5 \%}$ & $1960.5(8)_{+1.3 \%}^{+2.4 \%}$ \\
\hline$\overline{\mathrm{MS}}$ (FJTS) & $2181.3(8)_{-7.3 \%}^{+0.7 \%}$ & $2043.9(9)_{+1.3 \%}^{+0.8 \%}$ & $2150.5(6)_{-10.2 \%}^{+2.0 \%}$ & $1993.2(9)_{-9.3 \%}^{+2.8 \%}$ \\
\hline OS1 & $2175.5(8)$ & $2040.6(9)_{+0.1 \%}^{-0.1 \%}$ & $2104.4(6)$ & $1969.4(8)_{+0.0 \%}^{-0.1 \%}$ \\
\hline OS2 & $2179.5(8)$ & $2042.1(9)_{+0.1 \%}^{-0.2 \%}$ & $2116.5(6)$ & $1972.7(8)_{+0.2 \%}^{-0.2 \%}$ \\
\hline OS12 & $2176.5(8)$ & $2040.4(9)_{+0.1 \%}^{-0.2 \%}$ & $2110.5(6)$ & $1970.4(8)_{+0.1 \%}^{-0.2 \%}$ \\
\hline BFMS & $2168.6(8)$ & $2037.3(8)_{+0.1 \%}^{-0.1 \%}$ & $2098.4(6)$ & $1967.0(8)_{+0.1 \%}^{-0.1 \%}$ \\
\hline
\end{tabular}

Table 13. LO and NLO integrated cross sections $\sigma[\mathrm{fb}]$ for the production of the light Higgs boson in VBF, pp $\rightarrow \mathrm{H}_{2} \mathrm{jj}+X$, for the THDM scenarios A1 and A2 in different renormalization schemes, with the OS12 scheme as input scheme (and full conversion of the input parameters into the other schemes). The scale variation (given in percent) corresponds to the scales $\mu=\mu_{0} / 2$ and $\mu=2 \mu_{0}$ with central scale $\mu_{0}=\left(M_{\mathrm{H}_{2}}+M_{\mathrm{H}_{1}}+M_{A_{0}}+2 M_{\mathrm{H}^{+}}\right) / 5$.
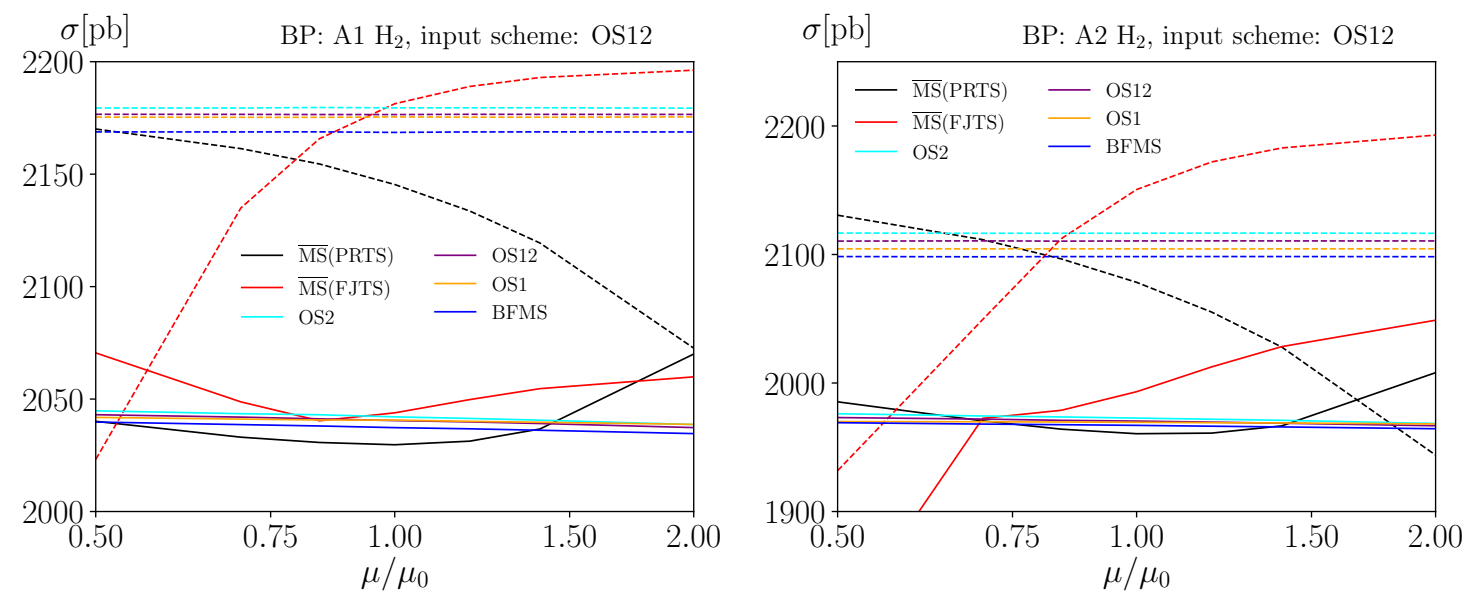

Figure 8. Scale dependence for light-Higgs-boson production in VBF, pp $\rightarrow \mathrm{H}_{2} \mathrm{jj}+X$, for the THDM benchmark scenarios A1 (left) and A2 (right) in different renormalization schemes, with the OS12 scheme as input scheme (and full conversion of the input parameters into the other schemes). LO results are shown as dashed, NLO as full lines; the central scale is set to $\mu_{0}=$ $\left(M_{\mathrm{H}_{2}}+M_{\mathrm{H}_{1}}+M_{A_{0}}+2 M_{\mathrm{H}^{+}}\right) / 5$.

gate any other benchmark scenarios. We plan to provide more detailed phenomenological results on VBF elsewhere.

\section{Conclusions}

Models with extended Higgs sectors are of prime importance for investigating the mechanism of electroweak symmetry breaking. Precision investigations of these models require the inclusion of higher-order corrections at least at NLO and thus renormalization. In particular, prescriptions for the renormalization of mixing angles in the scalar sector are needed. 
In this paper we have discussed a variety of renormalization prescriptions for scalar mixing angles with particular regard to symmetry, gauge independence, and numerical stability. In detail, we have considered and compared three types of renormalization schemes for mixing angles in the Higgs sector:

- $\overline{\mathrm{MS}}$ renormalization conditions for mixing angles are easy to implement. They depend, however, on the treatment of tadpoles and require care in view of gauge dependence, but have the benefit that the size of missing higher-order corrections can be investigated by renormalization scale variation.

- We have formulated on-shell renormalization conditions for the mixing angles in the Two-Higgs-Doublet Model and the Higgs-Singlet Extension of the Standard Model based on combinations of physical observables. More precisely, ratios of matrix elements or formfactors depending on the desired mixing angles only, deliver appropriate renormalization conditions. To obtain such ratios, it is often useful to introduce spurious particles with infinitesimal couplings, which do not change the physical theory.

- Rigid gauge invariance and/or the background-field method allow to introduce renormalization conditions for mixing angles in general theories.

We numerically studied and compared various renormalization conditions for mixing angles in the Two-Higgs-Doublet Model and the Higgs-Singlet Extension of the Standard Model for Higgs decays into four fermions and for Higgs-production in association with a vector boson or in vector-boson fusion. While renormalization schemes based on $\overline{\mathrm{MS}}$ subtraction tend to become unstable in delicate scenarios, in particular for heavy Higgsboson production in the Two-Higgs-Doublet Model, the proposed on-shell schemes and the schemes motivated by the background field method behave decently. They do not allow for a realistic estimate of missing higher-order corrections via scale variation, but instead the renormalization-scheme dependence can be investigated by comparing results obtained with different schemes after a consistent conversion of input parameters between the schemes. In general, one should avoid renormalization schemes that introduce artificial enhancement factors, e.g. for degenerate masses or small mixing angles.

Based on our study, we propose to use on-shell or symmetry-based schemes for the central predictions, as these turn out to be more robust. The reliability of the results can be checked by using two or more different on-shell schemes, where already the consistent parameter conversion between different schemes provides uncertainty estimates. Finally, schemes based on $\overline{\mathrm{MS}}$ renormalization can be used, with due care, to study scale uncertainties.

The schemes introduced in this paper are based on genuine on-shell conditions or simple symmetry principles. Thus, their generalization to higher orders is well-defined (similar to the $\overline{\mathrm{MS}}$ scheme), even if the explicit results for the counterterms might become more involved owing to reducible contributions. This is in contrast to some of the schemes proposed in the literature that rely on the form of the one-loop expressions.

The proposed schemes are not restricted to the considered models, but can be generalized to other extensions of the Standard Model, involving additional scalars, vector bosons, or fermions. 


\section{Acknowledgments}

Heidi Rzehak is gratefully acknowledged for useful discussions. The work of A.D. is supported by the German Science Foundation (DFG) under reference number DE 623/5-1, the work of S.D. by the DFG project DI 784/4-1. S.D. furthermore acknowledges support by the state of Baden-Württemberg through bwHPC and the DFG through grant no INST 39/963-1 FUGG. J.-N. Lang acknowledges support from the Swiss National Science Foundation (SNF) under contract BSCGI0-157722. The authors would like to express special thanks to the Mainz Institute for Theoretical Physics (MITP) for its hospitality and support.

\section{A Translation of conventions for the HSESM}

In order to treat the mixing between two Higgs bosons in a generic way, we deviate somewhat in our notation from the recent literature on the HSESM. Here, we collect translation rules for the fields and parameters from the HSESM formulation of previous works [28, 37]:

$$
\begin{aligned}
& \sigma=-S^{[28]} \sqrt{2}=\sigma^{[37]}, \\
& \eta_{1}=-\rho_{2}^{[28]}=h_{1}^{[37]}, \\
& \eta_{2}=\rho_{1}^{[28]}=h_{2}^{[37]} \text {, } \\
& v_{1}=-v_{s}^{[28]}=v_{1}^{[37]} \text {, } \\
& v_{2}=v^{[28]} \quad=v_{2}^{[37]} \text {, } \\
& H_{1}=H_{\mathrm{h}}^{[28]}=H^{[37]} \text {, } \\
& H_{2}=H_{1}^{[28]}=h^{[37]} \text {, } \\
& \alpha=\alpha^{[28]}+\frac{\pi}{2}=\alpha^{[37]}, \\
& c_{\alpha}=-s_{\alpha}^{[28]}=c_{\alpha}^{[37]} \text {, } \\
& s_{\alpha}=+c_{\alpha}^{[28]}=s_{\alpha}^{[37]} \text {, } \\
& \mu_{2}^{2}=-m_{1}^{2[28]}=\mu_{2}^{2[37]} \text {, } \\
& \mu_{1}^{2}=-m_{2}^{2[28]}=2 \mu_{1}^{2[37]}, \\
& \lambda_{2}=2 \lambda_{1}^{[28]}=\lambda_{2}^{[37]} \text {, } \\
& \lambda_{1}=2 \lambda_{2}^{[28]}=16 \lambda_{1}^{[37]} \text {, } \\
& \lambda_{3}=\lambda_{3}^{[28]}=2 \lambda_{12}^{[37]} \text {. }
\end{aligned}
$$

\section{B Translation of self-energies to the background-field method}

In section 3.3 we have derived renormalization conditions based on the background-field method which requires the evaluation of mixing and self-energies in this framework. Since the expressions differ from those in the conventional formalism, we provide the differences relevant for the renormalization of the mixing angles in the HSESM and the THDM using the following notation

$$
\Delta \Sigma^{X Y}\left(p^{2}\right)=\Sigma_{\mathrm{BFM}}^{\hat{X} \hat{Y}}\left(p^{2}\right)-\Sigma_{\text {conv. }}^{X Y}\left(p^{2}\right) .
$$

Some corresponding results in the SM can be found in ref. [69].

The one-loop tadpoles (one-point functions) do not differ between the BFM and the conventional formalism, although the individual tadpole contributions are not the same 
diagram by diagram. As a consequence also all tadpole contributions to the self-energies are the same in the BFM and the conventional formalism, and the differences reported in the following only result from 1PI contributions.

In order to use the mixing-angle renormalization based on the BFM, only the parameter counterterms have to be calculated in the BFM while the loop diagrams and the wave-function renormalization constants can be calculated in the conventional formalism (owing to gauge independence of the sum of a bare amplitude and its corresponding wavefunction counterterms). As far as parameter counterterms are determined from on-shell field renormalization constants, the latter have to be calculated in the BFM as well. To calculate the counterterms in the BFM formalism it is sufficient to combine the results for the differences (B.1) given in this appendix with the conventional self-energies.

In order to distinguish the electromagnetic coupling $\alpha_{\mathrm{em}}$ from the mixing angle $\alpha$, we label it with the index "em".

\section{B.1 Higgs sector of the HSESM}

In the HSESM the differences for the self-energies relevant for the renormalization of the mixing angle $\alpha$ read for $H_{i, j} \in\left\{H_{1}, H_{2}\right\}$

$$
\Delta \Sigma^{H_{i} H_{j}}\left(p^{2}\right)=\frac{\alpha_{\mathrm{em}}}{16 \pi c_{\mathrm{w}}^{2} s_{\mathrm{w}}^{2}} c^{H_{i} H_{j}}(s)\left[2 c_{\mathrm{w}}^{2} B_{0}\left(p^{2}, M_{\mathrm{W}}, M_{\mathrm{W}}\right)+B_{0}\left(p^{2}, M_{\mathrm{Z}}, M_{\mathrm{Z}}\right)\right],
$$

with

$$
\begin{aligned}
& c^{H_{1} H_{1}}(s)=2 s_{\alpha}^{2}\left(M_{\mathrm{H}_{1}}^{2}-p^{2}\right), \\
& c^{H_{1} H_{2}}(s)=c_{\alpha} s_{\alpha}\left(M_{\mathrm{H}_{1}}^{2}+M_{\mathrm{H}_{2}}^{2}-2 p^{2}\right), \\
& c^{H_{2} H_{2}}(s)=2 c_{\alpha}^{2}\left(M_{\mathrm{H}_{2}}^{2}-p^{2}\right) .
\end{aligned}
$$

For the pseudo-scalar and charged sector we have

$$
\begin{aligned}
\Delta \Sigma^{G_{0} G_{0}}\left(p^{2}\right)= & -\frac{\alpha_{\mathrm{em}}}{8 \pi c_{\mathrm{w}}^{2} s_{\mathrm{w}}^{2}} p^{2}\left[2 c_{\mathrm{w}}^{2} B_{0}\left(p^{2}, M_{\mathrm{W}}, M_{\mathrm{W}}\right)\right. \\
& \left.+c_{\alpha}^{2} B_{0}\left(p^{2}, M_{\mathrm{Z}}, M_{\mathrm{H}_{2}}\right)+s_{\alpha}^{2} B_{0}\left(p^{2}, M_{\mathrm{Z}}, M_{\mathrm{H}_{1}}\right)\right], \\
\Delta \Sigma^{G^{ \pm} G^{\mp}}\left(p^{2}\right)= & -\frac{\alpha_{\mathrm{em}}}{8 \pi s_{\mathrm{w}}^{2}} p^{2}\left[4 s_{\mathrm{w}}^{2} B_{0}\left(p^{2}, 0, M_{\mathrm{W}}\right)+\frac{4 c_{\mathrm{w}}^{4}-3 c_{\mathrm{w}}^{2}+1}{c_{\mathrm{w}}^{2}} B_{0}\left(p^{2}, M_{\mathrm{Z}}, M_{\mathrm{W}}\right)\right. \\
& \left.+c_{\alpha}^{2} B_{0}\left(p^{2}, M_{\mathrm{W}}, M_{\mathrm{H}_{2}}\right)+s_{\alpha}^{2} B_{0}\left(p^{2}, M_{\mathrm{W}}, M_{\mathrm{H}_{1}}\right)\right] .
\end{aligned}
$$

\section{B.2 Higgs sector of THDM}

In the THDM, the differences read for $H_{i, j} \in\left\{H_{1}, H_{2}\right\}$

$$
\begin{gathered}
\Delta \Sigma^{H_{1} H_{1}}\left(p^{2}\right)=\frac{\alpha_{\mathrm{em}}}{8 \pi c_{\mathrm{w}}^{2} s_{\mathrm{w}}^{2}}\left(M_{\mathrm{H}_{1}}^{2}-p^{2}\right)\left[c_{\alpha \beta}^{2} B_{0}\left(p^{2}, M_{\mathrm{Z}}, M_{\mathrm{Z}}\right)+s_{\alpha \beta}^{2} B_{0}\left(p^{2}, M_{\mathrm{Z}}, M_{A_{0}}\right)\right. \\
\left.+2 c_{\mathrm{w}}^{2}\left[c_{\alpha \beta}^{2} B_{0}\left(p^{2}, M_{\mathrm{W}}, M_{\mathrm{W}}\right)+s_{\alpha \beta}^{2} B_{0}\left(p^{2}, M_{\mathrm{W}}, M_{\mathrm{H}^{ \pm}}\right)\right]\right]
\end{gathered}
$$




$$
\begin{aligned}
& \Delta \Sigma^{H_{2} H_{2}}\left(p^{2}\right)=\frac{\alpha_{\mathrm{em}}}{8 \pi c_{\mathrm{w}}^{2} s_{\mathrm{w}}^{2}}\left(M_{\mathrm{H}_{2}}^{2}-p^{2}\right)\left[s_{\alpha \beta}^{2} B_{0}\left(p^{2}, M_{\mathrm{Z}}, M_{\mathrm{Z}}\right)+c_{\alpha \beta}^{2} B_{0}\left(p^{2}, M_{\mathrm{Z}}, M_{A_{0}}\right)\right. \\
& \left.+2 c_{\mathrm{w}}^{2}\left[s_{\alpha \beta}^{2} B_{0}\left(p^{2}, M_{\mathrm{W}}, M_{\mathrm{W}}\right)+c_{\alpha \beta}^{2} B_{0}\left(p^{2}, M_{\mathrm{W}}, M_{\mathrm{H}^{ \pm}}\right)\right]\right], \\
& \Delta \Sigma^{H_{1} H_{2}}\left(p^{2}\right)=-\frac{\alpha_{\mathrm{em}}}{16 \pi c_{\mathrm{w}}^{2} s_{\mathrm{w}}^{2}} c_{\alpha \beta} s_{\alpha \beta}\left(M_{\mathrm{H}_{1}}^{2}+M_{\mathrm{H}_{2}}^{2}-2 p^{2}\right)\left[B_{0}\left(p^{2}, M_{\mathrm{Z}}, M_{\mathrm{Z}}\right)-B_{0}\left(p^{2}, M_{\mathrm{Z}}, M_{A_{0}}\right)\right. \\
& \left.+2 c_{\mathrm{w}}^{2}\left[B_{0}\left(p^{2}, M_{\mathrm{W}}, M_{\mathrm{W}}\right)-B_{0}\left(p^{2}, M_{\mathrm{W}}, M_{\mathrm{H}^{ \pm}}\right)\right]\right],
\end{aligned}
$$

and

$$
c_{\alpha \beta}=\cos (\alpha-\beta), \quad s_{\alpha \beta}=\sin (\alpha-\beta) .
$$

For the pseudo-scalar fields we find

$$
\begin{aligned}
\Delta \Sigma^{G_{0} A_{0}}\left(p^{2}\right)= & \frac{\alpha_{\mathrm{em}}}{16 \pi c_{\mathrm{w}}^{2} s_{\mathrm{w}}^{2}} c_{\alpha \beta} s_{\alpha \beta}\left(M_{A_{0}}^{2}-2 p^{2}\right)\left[B_{0}\left(p^{2}, M_{\mathrm{Z}}, M_{\mathrm{H}_{1}}\right)-B_{0}\left(p^{2}, M_{\mathrm{Z}}, M_{\mathrm{H}_{2}}\right)\right], \\
\Delta \Sigma^{A_{0} A_{0}}\left(p^{2}\right)= & \frac{\alpha_{\mathrm{em}}}{8 \pi c_{\mathrm{w}}^{2} s_{\mathrm{w}}^{2}}\left(M_{A_{0}}^{2}-p^{2}\right)\left[2 c_{\mathrm{w}}^{2} B_{0}\left(p^{2}, M_{\mathrm{W}}, M_{\mathrm{H}^{ \pm}}\right)\right. \\
& \left.+s_{\alpha \beta}^{2} B_{0}\left(p^{2}, M_{\mathrm{Z}}, M_{\mathrm{H}_{1}}\right)+c_{\alpha \beta}^{2} B_{0}\left(p^{2}, M_{\mathrm{Z}}, M_{\mathrm{H}_{2}}\right)\right] \\
\Delta \Sigma^{G_{0} G_{0}}\left(p^{2}\right)= & -\frac{\alpha_{\mathrm{em}}}{8 \pi s_{\mathrm{w}}^{2} c_{\mathrm{w}}^{2}} p^{2}\left[2 c_{\mathrm{w}}^{2} B_{0}\left(p^{2}, M_{\mathrm{W}}, M_{\mathrm{W}}\right)\right. \\
& \left.+s_{\alpha \beta}^{2} B_{0}\left(p^{2}, M_{\mathrm{Z}}, M_{\mathrm{H}_{2}}\right)+c_{\alpha \beta}^{2} B_{0}\left(p^{2}, M_{\mathrm{Z}}, M_{\mathrm{H}_{1}}\right)\right]
\end{aligned}
$$

and for the charged ones we have

$$
\begin{aligned}
\Delta \Sigma^{G^{ \pm} H^{\mp}}\left(p^{2}\right)= & \frac{\alpha_{\mathrm{em}}}{8 \pi s_{\mathrm{w}}^{2}} c_{\alpha \beta} s_{\alpha \beta}\left(M_{\mathrm{H}^{ \pm}}^{2}-2 p^{2}\right)\left[B_{0}\left(p^{2}, M_{\mathrm{W}}, M_{\mathrm{H}_{1}}\right)-B_{0}\left(p^{2}, M_{\mathrm{W}}, M_{\mathrm{H}_{2}}\right)\right], \\
\Delta \Sigma^{H^{+} H^{-}}\left(p^{2}\right)= & \frac{\alpha_{\mathrm{em}}}{8 \pi s_{\mathrm{w}}^{2}}\left(M_{\mathrm{H}^{ \pm}}^{2}-p^{2}\right)\left[B_{0}\left(p^{2}, M_{\mathrm{W}}, M_{A_{0}}\right)\right. \\
& +4 s_{\mathrm{w}}^{2} B_{0}\left(p^{2}, 0, M_{\mathrm{H}^{ \pm}}\right)+\frac{\left(c_{\mathrm{w}}^{2}-s_{\mathrm{w}}^{2}\right)^{2}}{c_{\mathrm{w}}^{2}} B_{0}\left(p^{2}, M_{\mathrm{Z}}, M_{\mathrm{H}^{ \pm}}\right) \\
& \left.+s_{\alpha \beta}^{2} B_{0}\left(p^{2}, M_{\mathrm{W}}, M_{\mathrm{H}_{1}}\right)+c_{\alpha \beta}^{2} B_{0}\left(p^{2}, M_{\mathrm{W}}, M_{\mathrm{H}_{2}}\right)\right] \\
\Delta \Sigma^{G^{+} G^{-}}\left(p^{2}\right)= & -\frac{\alpha_{\mathrm{em}}}{8 \pi s_{\mathrm{w}}^{2}} p^{2}\left[4 s_{\mathrm{w}}^{2} B_{0}\left(p^{2}, 0, M_{\mathrm{W}}\right)+\frac{4 c_{\mathrm{w}}^{4}-3 c_{\mathrm{w}}^{2}+1}{c_{\mathrm{w}}^{2}} B_{0}\left(p^{2}, M_{\mathrm{Z}}, M_{\mathrm{W}}\right)\right. \\
& \left.+s_{\alpha \beta}^{2} B_{0}\left(p^{2}, M_{\mathrm{W}}, M_{\mathrm{H}_{2}}\right)+c_{\alpha \beta}^{2} B_{0}\left(p^{2}, M_{\mathrm{W}}, M_{\mathrm{H}_{1}}\right)\right] .
\end{aligned}
$$

\section{Tadpole contributions to scalar mixing and self-energies}

In general, the vertex functions contain explicit and implicit tadpole contributions. The explicit tadpole contributions, i.e. tadpole loop diagrams, result from the expansion of the effective action about a point that does not correspond to the stationary point. Implicit tadpole contributions or tadpole counterterms originate from tadpole terms in the Lagrangian or shifts in the fields. Since the treatment of tadpoles involves some freedom, 
and as we employ two $\overline{\mathrm{MS}}$ schemes differing in the treatment of tadpole counterterms, we briefly summarize their properties and provide, for completeness, the tadpole-counterterm expressions for mixing and self-energies that are needed in those renormalization schemes.

In the PRTS, the expansion is performed about the stationary point at the one-loop level, and the bare (squared) masses of the physical fields are defined as the coefficients of the terms quadratic in the corresponding fields. Moreover, we require vanishing mixing between the physical (mass-eigenstate) scalar fields, i.e. there are no implicit tadpoles in the mixing energies of these "physical fields". In the THDM we define the mixing angle $\beta$ from the ratio of the true vevs, $\tan \beta=v_{2} / v_{1}$. In this scheme, the tadpole terms result exclusively from tadpole counterterms in the Lagrangian and are absent, by definition, in two-point functions that involve only physical fields.

In the FJTS, all bare parameters are defined in terms of the bare parameters of the symmetric Lagrangian. The bare scalar fields are shifted, $H_{\mathrm{B}, i} \rightarrow H_{\mathrm{B}, i}+\Delta v_{i}$, so that the shifted fields describe excitations about the stationary point. The tadpole terms result exclusively from the shifts $\Delta v_{i}$ of the scalar fields. Tadpole terms appear in almost all vertex functions, apart from the one-point functions.

In the following we provide the implicit tadpole counterterms for the scalar self-energies and mixing energies in the THDM and HSESM both in the PRTS and the FJTS, using the following convention for the tadpole counterterms,

$$
\Sigma_{\text {tadpole }}^{H H^{\prime}}=t_{H H^{\prime}},
$$

corresponding to the second terms on the r.h.s. in (2.6). While in the PRTS the sum of the third and fourth terms in (2.6) is zero owing $\delta t_{\hat{H}}=-T^{\hat{H}}$, in the FJTS this condition must not necessary be fulfilled but is convenient.

\section{C.1 Tadpole counterterms in the FJTS}

In the FJTS the Feynman rules for two-point tadpole counterterms are easily obtained according to the formula

$$
t_{X Y}=-\frac{\delta t_{H_{1}}}{M_{\mathrm{H}_{1}}^{2}} C_{X Y \mathrm{H}_{1}}-\frac{\delta t_{H_{2}}}{M_{\mathrm{H}_{2}}^{2}} C_{X Y \mathrm{H}_{2}},
$$

where $X, Y$ are fields, and $\mathrm{i}_{X Y \mathrm{H}_{1}}, \mathrm{i}_{X Y \mathrm{H}_{2}}$ represent the couplings appearing in the Feynman rules involving those fields and either $\mathrm{H}_{1}$ or $\mathrm{H}_{2}$, respectively.

For example, in the HSESM we derive the following expressions:

$$
\begin{aligned}
& t_{H_{1} H_{1}}=3 \delta t_{H_{1}}\left(\frac{s_{\alpha}^{3}}{v_{2}}+\frac{c_{\alpha}^{3}}{v_{1}}\right)+\delta t_{H_{2}} s_{\alpha} c_{\alpha}\left(\frac{s_{\alpha}}{v_{2}}-\frac{c_{\alpha}}{v_{1}}\right)\left(1+\frac{2 M_{\mathrm{H}_{1}}^{2}}{M_{\mathrm{H}_{2}}^{2}}\right), \\
& t_{H_{2} H_{2}}=3 \delta t_{H_{2}}\left(\frac{c_{\alpha}^{3}}{v_{2}}-\frac{s_{\alpha}^{3}}{v_{1}}\right)+\delta t_{H_{1}} c_{\alpha} s_{\alpha}\left(\frac{c_{\alpha}}{v_{2}}+\frac{s_{\alpha}}{v_{1}}\right)\left(1+\frac{2 M_{\mathrm{H}_{2}}^{2}}{M_{\mathrm{H}_{1}}^{2}}\right), \\
& t_{H_{1} H_{2}}=c_{\alpha} s_{\alpha}\left[\delta t_{H_{1}}\left(\frac{s_{\alpha}}{v_{2}}-\frac{c_{\alpha}}{v_{1}}\right)\left(2+\frac{M_{\mathrm{H}_{2}}^{2}}{M_{\mathrm{H}_{1}}^{2}}\right)+\delta t_{H_{2}}\left(\frac{c_{\alpha}}{v_{2}}+\frac{s_{\alpha}}{v_{1}}\right)\left(2+\frac{M_{\mathrm{H}_{1}}^{2}}{M_{\mathrm{H}_{2}}^{2}}\right)\right], \\
& t_{G_{0} G_{0}}=t_{G^{ \pm} G^{ \pm}}=\frac{\delta t_{H_{1}} s_{\alpha}+\delta t_{H_{2}} c_{\alpha}}{v_{2}} .
\end{aligned}
$$


For the THDM the results for the tadpole counterterms in the FJTS can be found in appendix B of ref. [27].

\section{C.2 Tadpole counterterms in the PRTS}

In the HSESM the tadpole contributions in the PRTS for the scalar self-energies and mixing energies read:

$$
\begin{aligned}
& t_{H_{1} H_{1}}=t_{H_{2} H_{2}}=t_{H_{1} H_{2}}=0, \\
& t_{G_{0} G_{0}}=t_{G^{ \pm} G^{ \pm}}=\frac{\delta t_{H_{1}} s_{\alpha}+\delta t_{H_{2}} c_{\alpha}}{v_{2}},
\end{aligned}
$$

where $v_{2}=2 s_{\mathrm{w}} M_{\mathrm{W}} / e$.

In the THDM they can be extracted from refs. [27, 29] and read:

$$
\begin{aligned}
& t_{H_{1} H_{1}}=t_{H_{1} H_{2}}=t_{H_{2} H_{2}}=t_{A_{0} A_{0}}=t_{H^{ \pm} H^{ \pm}}=0, \\
& t_{G_{0} G_{0}}=t_{G^{ \pm} G^{ \pm}}=\frac{1}{v}\left(\delta t_{H_{1}} c_{\alpha \beta}-\delta t_{H_{2}} s_{\alpha \beta}\right), \\
& t_{G_{0} A_{0}}=t_{G^{ \pm} H^{ \pm}}=\frac{1}{v}\left(\delta t_{H_{1}} s_{\alpha \beta}+\delta t_{H_{2}} c_{\alpha \beta}\right),
\end{aligned}
$$

where $v=\sqrt{v_{1}^{2}+v_{2}^{2}}=2 s_{\mathrm{w}} M_{\mathrm{W}} / e$.

\section{Vertex corrections for on-shell schemes}

In this appendix we provide explicit results for relative vertex corrections entering the on-shell renormalization conditions introduced in section 3.2.2 for the THDM.

$$
\begin{aligned}
\delta_{H_{1} \nu_{1} \nu_{1}}=-\frac{\alpha_{\mathrm{em}} c_{\beta}}{4 \pi s_{\mathrm{w}}^{2} c_{\alpha}}[ & \frac{c_{\alpha \beta}}{2}\left(1-\frac{4 M_{\mathrm{W}}^{2}}{M_{\mathrm{H}_{1}}^{2}}\right) B_{0}\left(M_{\mathrm{H}_{1}}^{2}, M_{\mathrm{W}}, M_{\mathrm{W}}\right) \\
& +\frac{c_{\alpha \beta}}{4 c_{\mathrm{w}}^{2}}\left(1-\frac{4 M_{\mathrm{Z}}^{2}}{M_{\mathrm{H}_{1}}^{2}}\right) B_{0}\left(M_{\mathrm{H}_{1}}^{2}, M_{\mathrm{Z}}, M_{\mathrm{Z}}\right) \\
& -\frac{s_{\alpha \beta}}{2} t_{\beta} B_{0}\left(M_{\mathrm{H}_{1}}^{2}, M_{\mathrm{W}}, M_{\mathrm{H}^{ \pm}}\right)-\frac{s_{\alpha \beta} t_{\beta}}{4 c_{\mathrm{w}}^{2}} B_{0}\left(M_{\mathrm{H}_{1}}^{2}, M_{\mathrm{Z}}, M_{A_{0}}\right) \\
& +\left(-\frac{c_{\alpha}}{c_{\beta}}+\frac{2 M_{\mathrm{W}}^{2}}{M_{\mathrm{H}_{1}}^{2}} c_{\alpha \beta}\right) B_{0}\left(0, M_{\mathrm{W}}, 0\right) \\
& +\frac{1}{2 c_{\mathrm{w}}^{2}}\left(-\frac{c_{\alpha}}{c_{\beta}}+\frac{2 M_{\mathrm{Z}}^{2}}{M_{\mathrm{H}_{1}}^{2}} c_{\alpha \beta}\right) B_{0}\left(0, M_{\mathrm{Z}}, 0\right) \\
& -c_{\alpha \beta} M_{\mathrm{W}}^{2}\left(1-\frac{2 M_{\mathrm{W}}^{2}}{M_{\mathrm{H}_{1}}^{2}}\right) C_{0}\left(M_{\mathrm{H}_{1}}^{2}, 0,0, M_{\mathrm{W}}, M_{\mathrm{W}}, 0\right) \\
& -\frac{M_{\mathrm{Z}}^{2} c_{\alpha \beta}}{2 c_{\mathrm{w}}^{2}}\left(1-\frac{2 M_{\mathrm{Z}}^{2}}{M_{\mathrm{H}_{1}}^{2}}\right) C_{0}\left(M_{\mathrm{H}_{1}}^{2}, 0,0, M_{\mathrm{Z}}, M_{\mathrm{Z}}, 0\right) \\
& +s_{\alpha \beta} t_{\beta} M_{\mathrm{H}^{ \pm}}^{2} C_{0}\left(M_{\mathrm{H}_{1}}^{2}, 0,0, M_{\mathrm{W}}, M_{\mathrm{H}^{ \pm}}, 0\right) \\
& \left.+\frac{M_{A_{0}}^{2} s_{\alpha \beta} t_{\beta}}{2 c_{\mathrm{w}}^{2}} C_{0}\left(M_{\mathrm{H}_{1}}^{2}, 0,0, M_{\mathrm{Z}}, M_{A_{0}}, 0\right)\right]
\end{aligned}
$$




$$
\begin{aligned}
& \delta_{H_{2} \nu_{1} \nu_{1}}=\frac{\alpha_{\mathrm{em}} c_{\beta}}{4 \pi s_{\mathrm{W}}^{2} s_{\alpha}}\left[-\frac{s_{\alpha \beta}}{2}\left(1-\frac{4 M_{\mathrm{W}}^{2}}{M_{\mathrm{H}_{2}}^{2}}\right) B_{0}\left(M_{\mathrm{H}_{2}}^{2}, M_{\mathrm{W}}, M_{\mathrm{W}}\right)\right. \\
& -\frac{s_{\alpha \beta}}{4 c_{\mathrm{w}}^{2}}\left(1-\frac{4 M_{\mathrm{Z}}^{2}}{M_{\mathrm{H}_{2}}^{2}}\right) B_{0}\left(M_{\mathrm{H}_{2}}^{2}, M_{\mathrm{Z}}, M_{\mathrm{Z}}\right) \\
& -\frac{c_{\alpha \beta} t_{\beta}}{2} B_{0}\left(M_{\mathrm{H}_{2}}^{2}, M_{\mathrm{W}}, M_{\mathrm{H}^{ \pm}}\right)-\frac{c_{\alpha \beta} t_{\beta}}{4 c_{\mathrm{w}}^{2}} B_{0}\left(M_{\mathrm{H}_{2}}^{2}, M_{\mathrm{Z}}, M_{A_{0}}\right) \\
& +\left(\frac{s_{\alpha}}{c_{\beta}}-\frac{2 M_{\mathrm{W}}^{2}}{M_{\mathrm{H}_{2}}^{2}} s_{\alpha \beta}\right) B_{0}\left(0, M_{\mathrm{W}}, 0\right) \\
& +\frac{1}{2 c_{\mathrm{w}}^{2}}\left(\frac{s_{\alpha}}{c_{\beta}}-\frac{2 M_{\mathrm{Z}}^{2}}{M_{\mathrm{H}_{2}}^{2}} s_{\alpha \beta}\right) B_{0}\left(0, M_{\mathrm{Z}}, 0\right) \\
& +s_{\alpha \beta} M_{\mathrm{W}}^{2}\left(1-\frac{2 M_{\mathrm{W}}^{2}}{M_{\mathrm{H}_{2}}^{2}}\right) C_{0}\left(M_{\mathrm{H}_{2}}^{2}, 0,0, M_{\mathrm{W}}, M_{\mathrm{W}}, 0\right) \\
& +\frac{s_{\alpha \beta} M_{\mathrm{Z}}^{2}}{2 c_{\mathrm{w}}^{2}}\left(1-\frac{2 M_{\mathrm{Z}}^{2}}{M_{\mathrm{H}_{2}}^{2}}\right) C_{0}\left(M_{\mathrm{H}_{2}}^{2}, 0,0, M_{\mathrm{Z}}, M_{\mathrm{Z}}, 0\right) \\
& +c_{\alpha \beta} t_{\beta} M_{\mathrm{H}^{ \pm}}^{2} C_{0}\left(M_{\mathrm{H}_{2}}^{2}, 0,0, M_{\mathrm{W}}, M_{\mathrm{H}^{ \pm}}, 0\right) \\
& \left.+\frac{c_{\alpha \beta} t_{\beta} M_{A_{0}}^{2}}{2 c_{\mathrm{w}}^{2}} C_{0}\left(M_{\mathrm{H}_{2}}^{2}, 0,0, M_{\mathrm{Z}}, M_{A_{0}}, 0\right)\right], \\
& \delta_{A_{0} \nu_{1} \nu_{1}}=-\frac{\alpha_{\mathrm{em}}}{4 \pi s_{\mathrm{w}}^{2} t_{\beta}}\left[\frac{t_{\beta}}{2} B_{0}\left(M_{A_{0}}^{2}, M_{\mathrm{W}}, M_{\mathrm{H}^{ \pm}}\right)\right. \\
& -\frac{s_{\alpha \beta} c_{\alpha}}{4 c_{\mathrm{w}}^{2} c_{\beta}} B_{0}\left(M_{A_{0}}^{2}, M_{\mathrm{Z}}, M_{\mathrm{H}_{1}}\right)+\frac{c_{\alpha \beta} s_{\alpha}}{4 c_{\mathrm{w}}^{2} c_{\beta}} B_{0}\left(M_{A_{0}}^{2}, M_{\mathrm{Z}}, M_{\mathrm{H}_{2}}\right) \\
& -t_{\beta} B_{0}\left(0, M_{\mathrm{W}}, 0\right)-\frac{t_{\beta}}{2 c_{\mathrm{w}}^{2}} B_{0}\left(0, M_{\mathrm{Z}}, 0\right)-M_{\mathrm{H}^{ \pm}}^{2} t_{\beta} C_{0}\left(M_{A_{0}}^{2}, 0,0, M_{\mathrm{W}}, M_{\mathrm{H}^{ \pm}}, 0\right) \\
& +\frac{M_{\mathrm{H}_{1}}^{2} s_{\alpha \beta} c_{\alpha}}{2 c_{\mathrm{w}}^{2} c_{\beta}} C_{0}\left(M_{A_{0}}^{2}, 0,0, M_{\mathrm{Z}}, M_{\mathrm{H}_{1}}, 0\right) \\
& \left.-\frac{M_{\mathrm{H}_{2}}^{2} c_{\alpha \beta} s_{\alpha}}{2 c_{\mathrm{W}}^{2} c_{\beta}} C_{0}\left(M_{A_{0}}^{2}, 0,0, M_{\mathrm{Z}}, M_{\mathrm{H}_{2}}, 0\right)\right], \\
& \delta_{H_{1} \nu_{2} \nu_{2}}=-\frac{\alpha_{\mathrm{em}} s_{\beta}}{4 \pi s_{\mathrm{w}}^{2} s_{\alpha}}\left[\frac{c_{\alpha \beta}}{2}\left(1-\frac{4 M_{\mathrm{W}}^{2}}{M_{\mathrm{H}_{1}}^{2}}\right) B_{0}\left(M_{\mathrm{H}_{1}}^{2}, M_{\mathrm{W}}, M_{\mathrm{W}}\right)\right. \\
& +\frac{c_{\alpha \beta}}{4 c_{\mathrm{w}}^{2}}\left(1-\frac{4 M_{\mathrm{Z}}^{2}}{M_{\mathrm{H}_{1}}^{2}}\right) B_{0}\left(M_{\mathrm{H}_{1}}^{2}, M_{\mathrm{Z}}, M_{\mathrm{Z}}\right) \\
& +\frac{s_{\alpha \beta}}{2 t_{\beta}} B_{0}\left(M_{\mathrm{H}_{1}}^{2}, M_{\mathrm{W}}, M_{\mathrm{H}^{ \pm}}\right)+\frac{s_{\alpha \beta}}{4 c_{\mathrm{w}}^{2} t_{\beta}} B_{0}\left(M_{\mathrm{H}_{1}}^{2}, M_{\mathrm{Z}}, M_{A_{0}}\right) \\
& +\left(-\frac{s_{\alpha}}{s_{\beta}}+\frac{2 M_{\mathrm{W}}^{2}}{M_{\mathrm{H}_{1}}^{2}} c_{\alpha \beta}\right) B_{0}\left(0, M_{\mathrm{W}}, 0\right) \\
& +\frac{1}{2 c_{\mathrm{w}}^{2}}\left(-\frac{s_{\alpha}}{s_{\beta}}+\frac{2 M_{\mathrm{Z}}^{2}}{M_{\mathrm{H}_{1}}^{2}} c_{\alpha \beta}\right) B_{0}\left(0, M_{\mathrm{Z}}, 0\right) \\
& -M_{\mathrm{W}}^{2} c_{\alpha \beta}\left(1-\frac{2 M_{\mathrm{W}}^{2}}{M_{\mathrm{H}_{1}}^{2}}\right) C_{0}\left(M_{\mathrm{H}_{1}}^{2}, 0,0, M_{\mathrm{W}}, M_{\mathrm{W}}, 0\right) \\
& -\frac{M_{\mathrm{Z}}^{2} c_{\alpha \beta}}{2 c_{\mathrm{w}}^{2}}\left(1-\frac{2 M_{\mathrm{Z}}^{2}}{M_{\mathrm{H}_{1}}^{2}}\right) C_{0}\left(M_{\mathrm{H}_{1}}^{2}, 0,0, M_{\mathrm{Z}}, M_{\mathrm{Z}}, 0\right) \\
& -\frac{s_{\alpha \beta} M_{\mathrm{H}^{ \pm}}^{2}}{t_{\beta}} C_{0}\left(M_{\mathrm{H}_{1}}^{2}, 0,0, M_{\mathrm{W}}, M_{\mathrm{H}^{ \pm}}, 0\right) \\
& \left.-\frac{M_{A_{0}}^{2} s_{\alpha \beta}}{2 c_{\mathrm{w}}^{2} t_{\beta}} C_{0}\left(M_{\mathrm{H}_{1}}^{2}, 0,0, M_{\mathrm{Z}}, M_{A_{0}}, 0\right)\right] \text {, }
\end{aligned}
$$




$$
\begin{aligned}
\delta_{H_{2} \nu_{2} \nu_{2}}=-\frac{\alpha_{\mathrm{em}} s_{\beta}}{4 \pi s_{\mathrm{w}}^{2} c_{\alpha}}[ & -\frac{s_{\alpha \beta}}{2}\left(1-\frac{4 M_{\mathrm{W}}^{2}}{M_{\mathrm{H}_{2}}^{2}}\right) B_{0}\left(M_{\mathrm{H}_{2}}^{2}, M_{\mathrm{W}}, M_{\mathrm{W}}\right) \\
& -\frac{s_{\alpha \beta}}{4 c_{\mathrm{w}}^{2}}\left(1-\frac{4 M_{\mathrm{Z}}^{2}}{M_{\mathrm{H}_{2}}^{2}}\right) B_{0}\left(M_{\mathrm{H}_{2}}^{2}, M_{\mathrm{Z}}, M_{\mathrm{Z}}\right) \\
& +\frac{c_{\alpha \beta}}{2 t_{\beta}} B_{0}\left(M_{\mathrm{H}_{2}}^{2}, M_{\mathrm{W}}, M_{\mathrm{H}^{ \pm}}\right)+\frac{c_{\alpha \beta}}{4 c_{\mathrm{w}}^{2} t_{\beta}} B_{0}\left(M_{\mathrm{H}_{2}}^{2}, M_{\mathrm{Z}}, M_{A_{0}}\right) \\
& -\left(\frac{c_{\alpha}}{s_{\beta}}+\frac{2 M_{\mathrm{W}}^{2}}{M_{\mathrm{H}_{2}}^{2}} s_{\alpha \beta}\right) B_{0}\left(0, M_{\mathrm{W}}, 0\right) \\
& -\frac{1}{2 c_{\mathrm{w}}^{2}}\left(\frac{c_{\alpha}}{s_{\beta}}+\frac{2 M_{\mathrm{Z}}^{2}}{M_{\mathrm{H}_{2}}^{2}} s_{\alpha \beta}\right) B_{0}\left(0, M_{\mathrm{Z}}, 0\right) \\
& +M_{\mathrm{W}}^{2} s_{\alpha \beta}\left(1-\frac{2 M_{\mathrm{W}}^{2}}{M_{\mathrm{H}_{2}}^{2}}\right) C_{0}\left(M_{\mathrm{H}_{2}}^{2}, 0,0, M_{\mathrm{W}}, M_{\mathrm{W}}, 0\right) \\
& +\frac{M_{\mathrm{Z}}^{2} s_{\alpha \beta}}{2 c_{\mathrm{W}}^{2}}\left(1-\frac{2 M_{\mathrm{Z}}^{2}}{M_{\mathrm{H}_{2}}^{2}}\right) C_{0}\left(M_{\mathrm{H}_{2}}^{2}, 0,0, M_{\mathrm{Z}}, M_{\mathrm{Z}}, 0\right) \\
& -\frac{M_{\mathrm{H}^{ \pm}}^{2} c_{\alpha \beta}}{t_{\beta}} C_{0}\left(M_{\mathrm{H}_{2}}^{2}, 0,0, M_{\mathrm{W}}, M_{\mathrm{H}^{ \pm}}, 0\right) \\
& \left.-\frac{M_{A_{0}}^{2} c_{\alpha \beta}}{2 c_{\mathrm{w}}^{2} t_{\beta}} C_{0}\left(M_{\mathrm{H}_{2}}^{2}, 0,0, M_{\mathrm{Z}}, M_{A_{0}}, 0\right)\right], \\
\delta_{A_{0} \nu_{2} \nu_{2}}=-\frac{\alpha_{\mathrm{em}} t_{\beta}}{4 \pi s_{\mathrm{w}}^{2}}[ & \frac{1}{2 t_{\beta}} B_{0}\left(M_{A_{0}}^{2}, M_{\mathrm{W}}, M_{\mathrm{H}^{ \pm}}\right) \\
& +\frac{s_{\alpha \beta} s_{\alpha}}{4 c_{\mathrm{w}}^{2} s_{\beta}} B_{0}\left(M_{A_{0}}^{2}, M_{\mathrm{Z}}, M_{\mathrm{H}_{1}}\right)+\frac{c_{\alpha \beta} c_{\alpha}}{4 c_{\mathrm{w}}^{2} s_{\beta}} B_{0}\left(M_{A_{0}}^{2}, M_{\mathrm{Z}}, M_{\mathrm{H}_{2}}\right) \\
& -\frac{1}{t_{\beta}} B_{0}\left(0, M_{\mathrm{W}}, 0\right)-\frac{1}{2 c_{\mathrm{w}}^{2} t_{\beta}} B_{0}\left(0, M_{\mathrm{Z}}, 0\right) \\
& -\frac{M_{\mathrm{H}^{ \pm}}^{2}}{t_{\beta}} C_{0}\left(M_{A_{0}}^{2}, 0,0, M_{\mathrm{W}}, M_{\mathrm{H}^{ \pm}}, 0\right) \\
& -\frac{M_{\mathrm{H}_{1}}^{2} s_{\alpha \beta} s_{\alpha}}{2 c_{\mathrm{W}}^{2} s_{\beta}} C_{0}\left(M_{A_{0}}^{2}, 0,0, M_{\mathrm{H}_{1}}, M_{\mathrm{Z}}, 0\right) \\
& \left.-\frac{M_{\mathrm{H}_{2}}^{2} c_{\alpha \beta} c_{\alpha}}{2 c_{\mathrm{w}}^{2} s_{\beta}} C_{0}\left(M_{A_{0}}^{2}, 0,0, M_{\mathrm{H}_{2}}, M_{\mathrm{Z}}, 0\right)\right] . \\
&
\end{aligned}
$$

For the $B_{0}$ and $C_{0}$ functions we use the conventions of ref. [9].

\section{E Background-field Ward identities}

In the BFM, the invariance of the effective action under background gauge transformations gives rise to simple Ward identities (WIs) for the vertex functions of the background fields [34]. These WIs depend on the treatment of tadpoles. ${ }^{19}$

In general, the generating functional of vertex functions $\Gamma$ is related to the generating functional $T_{\mathrm{c}}$ of connected Green functions via the Legendre transformation

$$
T_{\mathrm{c}}\left[\left\{J_{\hat{X}}\right\}\right]=\mathrm{i} \sum_{\hat{X}} \int \mathrm{d}^{4} x J_{\hat{X}}(x) \hat{X}(x)+\mathrm{i} \Gamma[\{\hat{X}\}],
$$

\footnotetext{
${ }^{19}$ In this appendix the parameters $M_{\mathrm{Z}}, c_{\mathrm{W}}, s_{\mathrm{w}}$ should be understood as bare parameters and the WIs are those for bare vertex functions.
} 
where

$$
\frac{\delta T_{\mathrm{c}}}{\mathrm{i} \delta J_{\hat{X}}(x)}=\hat{X}(x) \quad \text { or } \quad \frac{\delta \Gamma}{\delta \hat{X}(x)}=-J_{\hat{X}}(x) .
$$

The arguments of $\Gamma$ related to the various (possibly shifted) fields of the theory are denoted by $\hat{X}$ and the corresponding arguments of $T_{\mathrm{c}}$ by $J_{\hat{X}}$. As usual, the connected 2-point Green functions are the inverse of the 2-point vertex functions

$$
\frac{\delta^{2} T_{\mathrm{c}}}{\mathrm{i} \delta J_{\hat{X}}(x) \mathrm{i} \delta J_{\hat{Y}}(y)}=-\left(\frac{\delta^{2} \mathrm{i} \Gamma}{\delta \hat{X}(x) \delta \hat{Y}(y)}\right)^{-1} .
$$

This relation implies, in particular, that the presence of tadpoles in the propagators is directly connected to the presence of tadpoles in the 2-point vertex functions. Accordingly, we define the self-energies $\Sigma$ as the higher-order contributions to the 2-point vertex functions (full inverse propagators) including all relevant tadpole contributions (cf. eq. (2.6)). The 1PI contribution to the self-energies $\Sigma_{1 \mathrm{PI}}$ includes only the first term on the r.h.s. of (2.6).

\section{E.1 A Standard Model example}

We illustrate the influence of the tadpole treatment on the BFM WIs using an example for the SM. Invariance of the effective action $\Gamma$ under background gauge transformations related to the parameter $\hat{\theta}^{Z}$ yields in general

$$
\begin{aligned}
0=\delta_{\hat{\theta}^{Z}} \Gamma= & -\partial_{\mu}^{x} \frac{\delta \Gamma}{\delta \hat{Z}_{\mu}(x)}[\{\hat{X}\}]-\frac{e}{2 c_{\mathrm{w}} s_{\mathrm{w}}}(\bar{v}+\hat{H}(x)) \frac{\delta \Gamma}{\delta \hat{\chi}(x)}[\{\hat{X}\}] \\
& +\frac{e}{2 c_{\mathrm{w}} s_{\mathrm{w}}} \hat{\chi}(x) \frac{\delta \Gamma}{\delta \hat{H}(x)}[\{\hat{X}\}]+\ldots,
\end{aligned}
$$

where $\{\hat{X}\}$ represents the set of all background fields of the SM and we suppressed some terms that are irrelevant in the following. Here, $\bar{v}+\hat{H}(x)$ denotes the decomposition of the Higgs-boson field into a constant vev $\bar{v}$ and the field excitation $\hat{H}(x)$, which will be made more precise for the different renormalization schemes.

The WIs are obtained by taking derivatives of (E.4) with respect to some background fields and evaluating at specific field values $\hat{X}(x)=\overline{\hat{X}}$. Differentiating for instance with respect to the would-be Goldstone-boson field $\hat{\chi}$ yields

$$
\begin{aligned}
0= & -\partial_{\mu}^{x} \frac{\delta^{2} \Gamma}{\delta \hat{Z}_{\mu}(x) \delta \hat{\chi}(y)}[\{\overline{\hat{X}}\}]-\frac{e}{2 c_{\mathrm{w}} s_{\mathrm{w}}}(\bar{v}+\overline{\hat{H}}) \frac{\delta^{2} \Gamma}{\delta \hat{\chi}(x) \delta \hat{\chi}(y)}[\{\overline{\hat{X}}\}] \\
& +\frac{e}{2 c_{\mathrm{w}} s_{\mathrm{w}}} \delta(x-y) \frac{\delta \Gamma}{\delta \hat{H}(x)}[\{\hat{\hat{X}}\}]+\frac{e}{2 c_{\mathrm{w}} s_{\mathrm{w}}} \overline{\hat{\chi}} \frac{\delta^{2} \Gamma}{\delta \hat{H}(x) \delta \hat{\chi}(y)}[\{\overline{\hat{X}}\}]+\ldots
\end{aligned}
$$

Expanding the vertex functional about the point $\hat{X}=\overline{\hat{X}}=0$ for $\bar{v}=v_{\mathrm{B}}$, i.e. for vanishing fields and without any extra shift in the fields, the identity (E.5) turns into

$$
0=-\partial_{\mu}^{x} \frac{\delta^{2} \Gamma}{\delta \hat{Z}_{\mu}(x) \delta \hat{\chi}(y)}[\{0\}]-\frac{e}{2 c_{\mathrm{w}} s_{\mathrm{w}}} v_{\mathrm{B}} \frac{\delta^{2} \Gamma}{\delta \hat{\chi}(x) \delta \hat{\chi}(y)}[\{0\}]+\frac{e}{2 c_{\mathrm{w}} s_{\mathrm{w}}} \delta(x-y) \frac{\delta \Gamma}{\delta \hat{H}(x)}[\{0\}] .
$$


Transforming to momentum space, expanding to one-loop order and using $M_{\mathrm{Z}}=$ $e v /\left(2 c_{\mathrm{w}} s_{\mathrm{w}}\right)$ implies for the one-loop contributions ${ }^{20}$

$$
0=p^{2} \Sigma_{1 \mathrm{PI}}^{\hat{Z} \hat{\hat{\chi}}}\left(p^{2}\right)-\mathrm{i} M_{\mathrm{Z}} \Sigma_{1 \mathrm{PI}}^{\hat{\chi} \hat{\chi}}\left(p^{2}\right)+\mathrm{i} \frac{e}{2 c_{\mathrm{w}} s_{\mathrm{w}}} T^{\hat{H}},
$$

which reproduces the WI (30) of ref. [34]. The Legendre transform (E.2) yields in this case

$$
\left.\frac{\delta T_{\mathrm{c}}}{\mathrm{i} \delta J_{\hat{X}}(x)}\right|_{\hat{Y}=0}=\hat{X}(x)=0
$$

i.e. the connected one-point functions, the explicit tadpoles, vanish and consequently the vertex functions are one-particle irreducible (1PI) as indicated in (E.7). Moreover, no implicit tadpoles occur since we expand about the bare vacuum. Note, however, that the so-defined vertex functions do not correspond to a generating functional of connected Green functions for vanishing sources and cannot be used to calculate the $S$-matrix in a straight-forward way unless additional tadpole contributions are included properly.

In the case of spontaneous symmetry breaking, the correct generating functional of connected Green functions is related to the vertex functional at the stationary point $\overline{\hat{X}}_{\mathrm{s}}$, defined by

$$
\frac{\delta \Gamma}{\delta \hat{X}}\left[\left\{\hat{\hat{X}}_{\mathrm{s}}\right\}\right]=0
$$

which according to (E.2) corresponds to $J_{\hat{X}}(x)=0$. On the other hand, (E.9) implies that all explicit tadpoles vanish. The expansion about the stationary point can be implemented in different ways as detailed in the following.

In the PRTS, the fields are expanded about the one-loop vev $\bar{v}=v$. The vertex functions are defined at the stationary point $\overline{\hat{X}}_{\mathrm{s}} \equiv 0$ for all fields, and consequently all explicit tadpoles vanish. The WI (E.5) becomes

$$
0=-\partial_{\mu}^{x} \frac{\delta^{2} \Gamma}{\delta \hat{Z}_{\mu}(x) \delta \hat{\chi}(y)}[\{0\}]-\frac{e v}{2 c_{\mathrm{w}} s_{\mathrm{w}}} \frac{\delta^{2} \Gamma}{\delta \hat{\chi}(x) \delta \hat{\chi}(y)}[\{0\}] .
$$

Transforming to momentum space implies for the one-loop self-energies

$$
0=p^{2} \Sigma^{\hat{Z}} \hat{\chi}\left(p^{2}\right)-\mathrm{i} M_{\mathrm{Z}} \Sigma^{\hat{\chi} \hat{\chi}}\left(p^{2}\right) .
$$

Splitting the self-energies into irreducible parts and implicit tadpole terms results in

$$
\begin{aligned}
0 & =p^{2} \Sigma_{1 \mathrm{PI}}^{\hat{Z} \hat{\chi}}\left(p^{2}\right)-\mathrm{i} M_{\mathrm{Z}}\left[\Sigma_{1 \mathrm{PI}}^{\hat{\chi} \hat{\chi}}\left(p^{2}\right)+\frac{\delta t_{\hat{H}}}{v}\right] \\
& =p^{2} \Sigma_{1 \mathrm{PI}}^{\hat{Z} \hat{\chi}}\left(p^{2}\right)-\mathrm{i} M_{\mathrm{Z}} \Sigma_{1 \mathrm{PI}}^{\hat{\chi} \hat{\chi}}\left(p^{2}\right)+\mathrm{i} \frac{e}{2 c_{\mathrm{w}} s_{\mathrm{w}}} T^{\hat{H}} .
\end{aligned}
$$

\footnotetext{
${ }^{20}$ For the definition of the Lorentz decomposition of the self-energies we use the conventions of ref. [34]. Note, however, that at variance with ref. [34], where all self-energies $\Sigma$ are 1PI, we use self-energies $\Sigma$ based on complete 2-point functions (cf. eq. (2.6)) and denote their 1PI parts by $\Sigma_{1 \mathrm{PI}}$.
} 
In the last step we used the fact that the Higgs tadpole is cancelled by the corresponding counterterm (implicit tadpole), i.e. $\delta t_{\hat{H}}=-T^{\hat{H}}$. The last line of eq. (E.12) coincides with (E.7) and the WI (30) in ref. [34].

In the FJTS, two different approaches can be used. In the first approach, which is our default, the fields are expanded about the stationary point, but the full vev consists of its tree-level part $v_{\mathrm{B}}$ and the field shift $\Delta v$,

$$
v=v_{\mathrm{B}}+\Delta v .
$$

Attributing this shift to the vev, the stationary point corresponds to $\overline{\hat{X}}_{\mathrm{s}}=0$ for all fields. All explicit tadpoles vanish, but owing to the shift in (E.13) extra terms proportional to $\Delta v$ appear in the WI, and (E.5) becomes

$$
0=-\partial_{\mu}^{x} \frac{\delta^{2} \Gamma}{\delta \hat{Z}_{\mu}(x) \delta \hat{\chi}(y)}[\{0\}]-\frac{e}{2 c_{\mathrm{w}} s_{\mathrm{w}}}\left(v_{\mathrm{B}}+\Delta v\right) \frac{\delta^{2} \Gamma}{\delta \hat{\chi}(x) \delta \hat{\chi}(y)}[\{0\}] .
$$

Transforming to momentum space and expanding to one-loop order yields

$$
0=p^{2} \Sigma^{\hat{Z}} \hat{\chi}\left(p^{2}\right)-\mathrm{i} M_{\mathrm{Z}} \Sigma \hat{\chi} \hat{\chi}\left(p^{2}\right)-\mathrm{i} M_{\mathrm{Z}} p^{2} \frac{\Delta v}{v_{\mathrm{B}}} .
$$

Upon a perturbative expansion and transformation to momentum space, the condition (E.9) for $\overline{\hat{X}}_{\mathrm{s}}=0$ fixes $\Delta v$ :

$$
\Delta v=-\frac{\delta t_{\hat{H}}}{M_{\mathrm{H}}^{2}}=\frac{T^{\hat{H}}}{M_{\mathrm{H}}^{2}} .
$$

Splitting the self-energies into irreducible parts and implicit tadpole terms results in

$$
\begin{aligned}
0 & =p^{2}\left[\Sigma_{1 \mathrm{PI}}^{\hat{Z} \hat{x}}\left(p^{2}\right)+\mathrm{i} M_{\mathrm{Z}} \frac{\Delta v}{v_{\mathrm{B}}}\right]-\mathrm{i} M_{\mathrm{Z}}\left[\Sigma_{1 \mathrm{PI}}^{\hat{\chi} \hat{\chi}}\left(p^{2}\right)-M_{\mathrm{H}}^{2} \frac{\Delta v}{v_{\mathrm{B}}}\right]-\mathrm{i} M_{\mathrm{Z}} p^{2} \frac{\Delta v}{v_{\mathrm{B}}} \\
& =p^{2} \Sigma_{1 \mathrm{PI}}^{\hat{Z} \hat{\hat{X}}}\left(p^{2}\right)-\mathrm{i} M_{\mathrm{Z}} \Sigma_{1 \mathrm{PI}}^{\hat{\hat{\chi}} \hat{\chi}}\left(p^{2}\right)+\mathrm{i} \frac{e}{2 c_{\mathrm{w}} s_{\mathrm{w}}} T^{\hat{H}} .
\end{aligned}
$$

Thus, in terms of irreducible self-energies we again recover (E.7), i.e. the WI (30) in ref. [34].

Alternatively, the FJTS can be used without the shift $\Delta v$, i.e. for $\bar{v}=v_{\mathrm{B}}$. Then, the stationary point of the vertex functional is determined from (E.9), so that

$$
0=\frac{\delta \Gamma}{\delta \hat{H}(x)}\left[\left\{\overline{\hat{X}}_{\mathrm{s}}\right\}\right]=\frac{\delta \Gamma}{\delta \hat{H}(x)}[\{0\}]+\int \mathrm{d}^{4} z \frac{\delta^{2} \Gamma}{\delta \hat{H}(x) \delta \hat{H}(z)}[\{0\}] \overline{\hat{H}}_{\mathrm{s}}(z)+\mathcal{O}\left(\overline{\hat{H}}_{\mathrm{s}}^{2}\right) .
$$

Together with (E.3) and after a perturbative expansion of the propagator this yields

$$
\begin{aligned}
\overline{\hat{H}}_{\mathrm{s}}(x) & =\mathrm{i} \int \mathrm{d}^{4} z \frac{\delta T_{\mathrm{c}}}{\mathrm{i} \delta J_{\hat{H}}(x) \mathrm{i} \delta J_{\hat{H}}(z)}[\{0\}] \frac{\delta \Gamma}{\delta \hat{H}(z)}[\{0\}]+\mathcal{O}\left(\overline{\hat{H}}_{\mathrm{s}}^{2}\right), \\
& =\mathrm{i} \frac{\mathrm{i}}{-M_{\mathrm{H}}^{2}} T^{\hat{H}}+\mathcal{O}\left(\hat{\hat{H}}_{\mathrm{s}}^{2}\right)
\end{aligned}
$$

where the second line results after transformation to momentum space. Thus, $\overline{\hat{H}}_{\mathrm{s}}$ corresponds to the tadpole with external propagator attached, so that we get at NLO

$$
\overline{\hat{H}}_{\mathrm{s}}=\frac{T^{\hat{H}}}{M_{\mathrm{H}}^{2}},
$$


while

$$
\overline{\hat{X}}_{\mathrm{s}}=0, \quad \hat{X} \neq \hat{H} .
$$

In this formulation, (E.5) turns into

$$
0=-\partial_{\mu}^{x} \frac{\delta^{2} \Gamma}{\delta \hat{Z}_{\mu}(x) \delta \hat{\chi}(y)}\left[\left\{\overline{\hat{X}}_{\mathrm{s}}\right\}\right]-\frac{e}{2 c_{\mathrm{w}} s_{\mathrm{w}}}\left(v_{\mathrm{B}}+\overline{\hat{H}}_{\mathrm{s}}\right) \frac{\delta^{2} \Gamma}{\delta \hat{\chi}(x) \delta \hat{\chi}(y)}\left[\left\{\overline{\hat{X}}_{\mathrm{s}}\right\}\right] .
$$

While the WI (E.22) and the vertex functions $\frac{\delta^{2} \Gamma}{\delta \hat{X}(x) \delta \hat{Y}(y)}\left[\left\{\hat{X}_{\mathrm{s}}\right\}\right]$ appearing therein evaluated at the stationary point $\overline{\hat{X}}_{\mathrm{s}}$ do not contain explicit tadpoles, a perturbative expansion yields

$$
\begin{aligned}
\frac{\delta^{2} \Gamma}{\delta \hat{X}(x) \delta \hat{Y}(y)}\left[\left\{\overline{\hat{X}}_{\mathrm{s}}\right\}\right]= & \frac{\delta^{2} \Gamma}{\delta \hat{X}(x) \delta \hat{Y}(y)}[\{0\}] \\
& +\int \mathrm{d}^{4} z \frac{\delta^{3} \Gamma}{\delta \hat{X}(x) \delta \hat{Y}(y) \delta \hat{H}(z)}[\{0\}] \overline{\hat{H}}_{\mathrm{s}}(z)+\mathcal{O}\left(\hat{\hat{H}}_{\mathrm{s}}^{2}\right)
\end{aligned}
$$

where the terms $\mathcal{O}\left(\overline{\hat{H}}_{\mathrm{s}}^{2}\right)$ are beyond one-loop order. The first term on the r.h.s. of (E.23) delivers the two 1PI contributions appearing in (E.6), the second term corresponds to an explicit tadpole contribution to the $\hat{X} \hat{Y}$ self-energy. Thus, in terms of usual building blocks the vertex functions are composed of 1PI terms including contributions from explicit tadpoles. The corresponding WIs in momentum space are obtained upon replacing $\Delta v \rightarrow$ $T^{\hat{H}} / M_{\mathrm{H}}^{2}$ in (E.15) and (E.17), i.e. they are equivalent to those equations.

\section{E.2 A THDM example}

We give a second example ${ }^{21}$ that is relevant for the renormalization of the mixing angle $\beta$ in the THDM. Starting from the analogue of the WI (E.4) in the THDM and differentiating with respect to the physical pseudoscalar field $A_{0}$ yields when expanding about the stationary point:

$$
\begin{aligned}
0= & -\partial_{\mu}^{x} \frac{\delta^{2} \Gamma}{\delta \hat{Z}_{\mu}(x) \delta \hat{A}_{0}(y)}\left[\left\{\overline{\hat{X}}_{\mathrm{s}}\right\}\right]-\frac{e}{2 c_{\mathrm{w}} s_{\mathrm{w}}}\left(c_{\beta} v_{1}+s_{\beta} v_{2}\right) \frac{\delta^{2} \Gamma}{\delta \hat{G}_{0}(x) \delta \hat{A}_{0}(y)}\left[\left\{\hat{\hat{X}}_{\mathrm{s}}\right\}\right] \\
& +\frac{e}{2 c_{\mathrm{w}} s_{\mathrm{w}}}\left(s_{\beta} v_{1}-c_{\beta} v_{2}\right) \frac{\delta^{2} \Gamma}{\delta \hat{A}_{0}(x) \delta \hat{A}_{0}(y)}\left[\left\{\hat{\hat{X}}_{\mathrm{s}}\right\}\right]+\ldots
\end{aligned}
$$

In the PRTS, where $v_{1}$ and $v_{2}$ are the one-loop vevs, $t_{\beta}=v_{2} / v_{1}$, and $\overline{\hat{X}}_{\mathrm{s}}=0$, this becomes

$$
0=-\partial_{\mu}^{x} \frac{\delta^{2} \Gamma}{\delta \hat{Z}_{\mu}(x) \hat{A}_{0}(y)}[\{0\}]-M_{\mathrm{Z}} \frac{\delta^{2} \Gamma}{\delta \hat{G}_{0}(x) \delta \hat{A}_{0}(y)}[\{0\}]
$$

where we used

$$
M_{\mathrm{Z}}=\frac{e}{2 c_{\mathrm{w}} s_{\mathrm{w}}} v=\frac{e}{2 c_{\mathrm{w}} s_{\mathrm{w}}}\left(c_{\beta} v_{1}+s_{\beta} v_{2}\right) .
$$

\footnotetext{
${ }^{21}$ For other examples of BFM Ward identities in the THDM see footnote 5 of ref. [29].
} 
Transforming to momentum space and setting $p^{2}=0$, this leads to

$$
0=\Sigma^{\hat{G}_{0} \hat{A}_{0}}(0),
$$

or in terms of $1 \mathrm{PI}$ mixing energies

$$
0=\Sigma_{1 \mathrm{PI}}^{\hat{G}_{0} \hat{A}_{0}}(0)+\frac{e}{2 M_{\mathrm{W}} s_{\mathrm{w}}}\left(s_{\alpha \beta} \delta t_{H_{1}}+c_{\alpha \beta} \delta t_{H_{2}}\right) .
$$

In the FJTS, on the other hand, where $v_{1}=v_{1 \mathrm{~B}}+\Delta v_{1}, v_{2}=v_{2 \mathrm{~B}}+\Delta v_{2}$, and $\overline{\hat{X}}_{\mathrm{s}}=0$, we find

$$
\begin{aligned}
0= & -\partial_{\mu}^{x} \frac{\delta^{2} \Gamma}{\delta \hat{Z}_{\mu}(x) \hat{A}_{0}(y)}[\{0\}]-M_{\mathrm{Z}}\left(1+c_{\beta} \frac{\Delta v_{1}}{v_{\mathrm{B}}}+s_{\beta} \frac{\Delta v_{2}}{v_{\mathrm{B}}}\right) \frac{\delta^{2} \Gamma}{\delta \hat{G}_{0}(x) \delta \hat{A}_{0}(y)}[\{0\}] \\
& +M_{\mathrm{Z}}\left(s_{\beta} \frac{\Delta v_{1}}{v_{\mathrm{B}}}-c_{\beta} \frac{\Delta v_{2}}{v_{\mathrm{B}}}\right) \frac{\delta^{2} \Gamma}{\delta \hat{A}_{0}(x) \delta \hat{A}_{0}(y)}[\{0\}],
\end{aligned}
$$

where we used

$$
M_{\mathrm{Z}}=\frac{e}{2 c_{\mathrm{w}} s_{\mathrm{w}}} v_{\mathrm{B}}=\frac{e}{2 c_{\mathrm{w}} s_{\mathrm{w}}}\left(c_{\beta} v_{1 \mathrm{~B}}+s_{\beta} v_{2 \mathrm{~B}}\right)
$$

and $c_{\beta}, s_{\beta}, t_{\beta}=v_{2 \mathrm{~B}} / v_{1 \mathrm{~B}}$ are bare parameters.

Transformation to momentum space, setting $p^{2}=0$ and using the explicit LO contributions to the vertex functions, results in

$$
0=\Sigma^{\hat{G}_{0} \hat{A}_{0}}(0)-\frac{1}{v_{\mathrm{B}}}\left(c_{\beta} \Delta v_{2}-s_{\beta} \Delta v_{1}\right) M_{A_{0}}^{2} .
$$

Splitting the mixing energy into 1PI parts and implicit counterterms and expressing $\Delta v_{i}$ by tadpole counterterms, we obtain again (E.28).

\section{F Parameter conversion tables}

In this appendix we give results for the parameter conversion from the on-shell input schemes OS and OS12 in the HSESM and THDM, respectively, to the other renormalization schemes in two different variants, as used for the results presented in section 4 . The two variants are full conversions, as explained in section 3.5, are equivalent at NLO, but differ in higher orders due to the tadpole counterterm scheme and the precise form of the input parameters. For the details see the caption of the tables in the following.

\section{F.1 HSESM scenarios of table 3}

In table 14 the results for the full conversion of parameters from the OS as input scheme to other schemes for the considered benchmark scenarios of table 3 are shown. No large conversion effects are observed, and the two different conversion variants mutually agree. 


\begin{tabular}{|c|c|c|c|c|c|c|c|c|}
\hline Conversion 1 & \multicolumn{2}{|c|}{ BHM200 $^{+}$} & \multicolumn{2}{|c|}{ BHM200 $^{-}$} & \multicolumn{2}{c|}{ BHM400 } & \multicolumn{2}{c|}{ BHM600 } \\
Scheme & $s_{\alpha}$ & $\frac{\lambda_{3}}{2}$ & $s_{\alpha}$ & $\frac{\lambda_{3}}{2}$ & $s_{\alpha}$ & $\frac{\lambda_{3}}{2}$ & $s_{\alpha}$ & $\frac{\lambda_{3}}{2}$ \\
\hline OS & 0.29 & 0.07 & -0.29 & -0.07 & 0.26 & 0.17 & 0.22 & 0.23 \\
\hline$\overline{\mathrm{MS}}$ (PRTS) & 0.302 & 0.073 & -0.304 & -0.073 & 0.267 & 0.175 & 0.226 & 0.236 \\
\hline$\overline{\mathrm{MS}}$ (FJTS) & 0.321 & 0.077 & -0.316 & -0.076 & 0.264 & 0.173 & 0.212 & 0.222 \\
\hline BFMS & 0.290 & 0.070 & -0.290 & -0.070 & 0.258 & 0.172 & 0.218 & 0.237 \\
\hline
\end{tabular}

\begin{tabular}{|c|c|c|c|c|c|c|c|c|}
\hline Conversion 2 & \multicolumn{2}{|c|}{ BHM200 } & \multicolumn{2}{c|}{ BHM200- $^{-}$} & \multicolumn{2}{c|}{ BHM400 } & \multicolumn{2}{c|}{ BHM600 } \\
Scheme & $s_{\alpha}$ & $\frac{\lambda_{3}}{2}$ & $s_{\alpha}$ & $\frac{\lambda_{3}}{2}$ & $s_{\alpha}$ & $\frac{\lambda_{3}}{2}$ & $s_{\alpha}$ & $\frac{\lambda_{3}}{2}$ \\
\hline OS & 0.29 & 0.07 & -0.29 & -0.07 & 0.26 & 0.17 & 0.22 & 0.23 \\
\hline$\overline{\mathrm{MS}}$ (PRTS) & 0.302 & 0.073 & -0.304 & -0.073 & 0.267 & 0.174 & 0.226 & 0.236 \\
\hline$\overline{\mathrm{MS}}$ (FJTS) & 0.319 & 0.077 & -0.315 & -0.076 & 0.264 & 0.172 & 0.212 & 0.222 \\
\hline BFMS & 0.290 & 0.070 & -0.290 & -0.070 & 0.258 & 0.172 & 0.220 & 0.238 \\
\hline
\end{tabular}

Table 14. Conversion of parameters from the OS scheme as input scheme to other renormalization schemes at the central scale $\mu=M_{\mathrm{H}_{2}}$ performed in the PRTS (upper table, used for the Higgs decays in section 4.1.1) and FJTS (lower table, used for Higgs-production processes in section 4.2.2) tadpole scheme. Besides the choice of tadpole counterterm scheme, also the choice of bare parameters for the matching differs. In the upper table $\tan \alpha_{\mathrm{B}}$ and $\lambda_{3}$ are used while in the lower one $\alpha_{\mathrm{B}}$ and $\tan \beta_{\mathrm{B}}$ are used.

\section{F.2 THDM scenarios of table 6}

In table 15 the corresponding conversion in the THDM from the OS12 as input scheme to other renormalization schemes is shown. As compared to the HSESM, the conversion effects for the scenarios in table 6 are more pronounced. Generically the conversion effects are $\lesssim 5 \%$ for the on-shell schemes and for the BFMS scheme, with the only exception being the OS1 scheme in scenario B1 with conversion effects of the order of $\sim 40 \%$. The conversion effects to the $\overline{\mathrm{MS}}$ schemes are typically larger than those to the on-shell schemes, and the conversion to $\overline{\mathrm{MS}}$ (FJTS) becomes perturbatively unstable (and thus fails completely) for scenarios B1 and B2 while for $\overline{\mathrm{MS}}$ (PRTS) only B2 is unstable.

Comparing the two conversions, the differences in the scenarios A1 and A2 to $\overline{\mathrm{MS}}(\mathrm{PRTS})\left(\overline{\mathrm{MS}}(\mathrm{FJTS})\right.$ ) amount to $13 \%(6 \%)$ and $1 \%(1 \%)$ for $c_{\alpha \beta}$ and $t_{\beta}$, respectively. The different conversions to the on-shell schemes and the BFMS scheme agree on the level of $4 \%$, with scenario B1 being again the only exception where we find a difference of $33 \%$ in the conversion of $t_{\beta}$.

Open Access. This article is distributed under the terms of the Creative Commons Attribution License (CC-BY 4.0), which permits any use, distribution and reproduction in any medium, provided the original author(s) and source are credited. 


\begin{tabular}{|c|c|c|c|c|c|c|c|c|}
\hline Conversion 1 & \multicolumn{2}{|c|}{ A1 } & \multicolumn{2}{c|}{$\mathrm{A} 2$} & \multicolumn{2}{c|}{$\mathrm{B} 1$} & \multicolumn{2}{c|}{$\mathrm{B} 2$} \\
Scheme & $c_{\alpha \beta}$ & $t_{\beta}$ & $c_{\alpha \beta}$ & $t_{\beta}$ & $c_{\alpha \beta}$ & $t_{\beta}$ & $c_{\alpha \beta}$ & $t_{\beta}$ \\
\hline OS12 & 0.1 & 2.0 & 0.2 & 2.0 & 0.15 & 4.5 & 0.3 & 3.0 \\
\hline$\overline{\mathrm{MS}}$ (PRTS) & 0.137 & 1.90 & 0.225 & 1.91 & 0.153 & 4.40 & 0.176 & 2.83 \\
\hline$\overline{\mathrm{MS}}$ (FJTS) & 0.090 & 1.93 & 0.157 & 1.91 & 0.061 & 3.82 & -0.031 & 3.70 \\
\hline OS1 & 0.102 & 1.92 & 0.205 & 1.91 & 0.208 & 3.46 & 0.309 & 2.87 \\
\hline OS2 & 0.096 & 2.02 & 0.195 & 2.03 & 0.145 & 4.61 & 0.298 & 3.02 \\
\hline BFMS & 0.110 & 1.91 & 0.209 & 1.92 & 0.149 & 4.42 & 0.323 & 2.82 \\
\hline
\end{tabular}

\begin{tabular}{|c|c|c|c|c|c|c|c|c|}
\hline $\begin{array}{c}\text { Conversion } 2 \\
\text { Scheme }\end{array}$ & \multicolumn{2}{|c|}{$\mathrm{A} 1$} & \multicolumn{2}{c|}{$\mathrm{A} 2$} & \multicolumn{2}{c|}{$\mathrm{B} 1$} & \multicolumn{2}{c|}{$\mathrm{B} 2$} \\
\hline OS12 & $c_{\alpha \beta}$ & $t_{\beta}$ & $c_{\alpha \beta}$ & $t_{\beta}$ & $c_{\alpha \beta}$ & $t_{\beta}$ & $c_{\alpha \beta}$ & $t_{\beta}$ \\
\hline$\overline{\mathrm{MS}}$ (PRTS) & 0.155 & 2.0 & 0.2 & 2.0 & 0.15 & 4.5 & 0.3 & 3.0 \\
\hline$\overline{\mathrm{MS}}$ (FJTS) & 0.089 & 1.93 & 0.148 & 1.89 & -0.015 & 2.34 & 0.049 & 3.22 \\
\hline OS1 & 0.102 & 1.92 & 0.207 & 1.91 & 0.199 & 4.61 & 0.308 & 2.89 \\
\hline OS2 & 0.093 & 2.02 & 0.193 & 2.02 & 0.147 & 4.44 & 0.299 & 3.01 \\
\hline BFMS & 0.116 & 1.92 & 0.214 & 1.92 & 0.151 & 4.44 & 0.319 & 2.83 \\
\hline
\end{tabular}

Table 15. Conversion of parameters from the OS12 scheme as input scheme to other renormalization schemes at the central scale $\mu_{0}$ performed in the PRTS (upper table, used for the Higgs decays in section 4.1.2) and FJTS (lower table, used for Higgs-production processes in sections 4.2.3 and 4.2.4) tadpole scheme. Besides the choice of tadpole counterterm scheme, also the bare parameters for the matching differs. In the upper table $\tan \alpha_{\mathrm{B}}$ and $\tan \beta_{\mathrm{B}}$ are used while in the lower one $\alpha_{\mathrm{B}}$ and $\beta_{\mathrm{B}}$ are used.

\section{References}

[1] CMS collaboration, Observation of a new boson at a mass of $125 \mathrm{GeV}$ with the CMS experiment at the LHC, Phys. Lett. B 716 (2012) 30 [arXiv:1207.7235] [INSPIRE].

[2] ATLAS collaboration, Observation of a new particle in the search for the Standard Model Higgs boson with the ATLAS detector at the LHC, Phys. Lett. B 716 (2012) 1 [arXiv: 1207.7214] [INSPIRE].

[3] J.F. Gunion and H.E. Haber, The CP conserving Two-Higgs-Doublet Model: the approach to the decoupling limit, Phys. Rev. D 67 (2003) 075019 [hep-ph/0207010] [INSPIRE].

[4] G.C. Branco, P.M. Ferreira, L. Lavoura, M.N. Rebelo, M. Sher and J.P. Silva, Theory and phenomenology of Two-Higgs-Doublet Models, Phys. Rept. 516 (2012) 1 [arXiv:1106. 0034] [INSPIRE].

[5] R.M. Schabinger and J.D. Wells, A minimal spontaneously broken hidden sector and its impact on Higgs boson physics at the large hadron collider, Phys. Rev. D 72 (2005) 093007 [hep-ph/0509209] [INSPIRE]. 
[6] B. Patt and F. Wilczek, Higgs-field portal into hidden sectors, hep-ph/0605188 [INSPIRE].

[7] M. Bowen, Y. Cui and J.D. Wells, Narrow trans-TeV Higgs bosons and $H \rightarrow$ hh decays: Two LHC search paths for a hidden sector Higgs boson, JHEP 03 (2007) 036 [hep-ph/0701035] [INSPIRE].

[8] A. Denner and T. Sack, Renormalization of the Quark Mixing Matrix, Nucl. Phys. B 347 (1990) 203 [INSPIRE].

[9] A. Denner, Techniques for calculation of electroweak radiative corrections at the one-loop level and results for W physics at LEP-200, Fortsch. Phys. 41 (1993) 307 [arXiv:0709.1075] [INSPIRE].

[10] P. Gambino, P.A. Grassi and F. Madricardo, Fermion mixing renormalization and gauge invariance, Phys. Lett. B 454 (1999) 98 [hep-ph/9811470] [InSPIRE].

[11] C. Balzereit, T. Mannel and B. Plümper, The renormalization group evolution of the CKM matrix, Eur. Phys. J. C 9 (1999) 197 [hep-ph/9810350] [INSPIRE].

[12] A. Pilaftsis, Gauge and scheme dependence of mixing matrix renormalization, Phys. Rev. D 65 (2002) 115013 [hep-ph/0203210] [INSPIRE].

[13] K.P.O. Diener and B.A. Kniehl, On mass shell renormalization of fermion mixing matrices, Nucl. Phys. B 617 (2001) 291 [hep-ph/0109110] [INSPIRE].

[14] B.A. Kniehl and A. Sirlin, Simple On-Shell Renormalization Framework for the Cabibbo-Kobayashi-Maskawa Matrix, Phys. Rev. D 74 (2006) 116003 [hep-th/0612033] [INSPIRE].

[15] B.A. Kniehl and A. Sirlin, A Novel Formulation of Cabibbo-Kobayashi-Maskawa Matrix Renormalization, Phys. Lett. B 673 (2009) 208 [arXiv:0901.0114] [INSPIRE].

[16] A. Denner, E. Kraus and M. Roth, Physical renormalization condition for the quark mixing matrix, Phys. Rev. D 70 (2004) 033002 [hep-ph/0402130] [INSPIRE].

[17] Y. Yamada, Gauge dependence of the on-shell renormalized mixing matrices, Phys. Rev. D 64 (2001) 036008 [hep-ph/0103046] [INSPIRE].

[18] P.H. Chankowski, S. Pokorski and J. Rosiek, Complete on-shell renormalization scheme for the minimal supersymmetric Higgs sector, Nucl. Phys. B 423 (1994) 437 [hep-ph/9303309] [INSPIRE].

[19] A. Dabelstein, The one loop renormalization of the MSSM Higgs sector and its application to the neutral scalar Higgs masses, Z. Phys. C 67 (1995) 495 [hep-ph/9409375] [INSPIRE].

[20] A. Freitas and D. Stöckinger, Gauge dependence and renormalization of tan beta in the MSSM, Phys. Rev. D 66 (2002) 095014 [hep-ph/0205281] [INSPIRE].

[21] N. Baro, F. Boudjema and A. Semenov, Automatised full one-loop renormalisation of the MSSM. I. The Higgs sector, the issue of $\tan \beta$ and gauge invariance, Phys. Rev. D 78 (2008) 115003 [arXiv: 0807 .4668] [INSPIRE].

[22] N. Baro and F. Boudjema, Automatised full one-loop renormalisation of the MSSM II: The chargino-neutralino sector, the sfermion sector and some applications, Phys. Rev. D 80 (2009) 076010 [arXiv:0906.1665] [INSPIRE].

[23] S. Kanemura, Y. Okada, E. Senaha and C.P. Yuan, Higgs coupling constants as a probe of new physics, Phys. Rev. D 70 (2004) 115002 [hep-ph/0408364] [INSPIRE]. 
[24] D. Lopez-Val and J. Solà, Neutral Higgs-pair production at Linear Colliders within the general 2HDM: Quantum effects and triple Higgs boson self-interactions, Phys. Rev. D 81 (2010) 033003 [arXiv:0908.2898] [INSPIRE].

[25] S. Kanemura, M. Kikuchi and K. Yagyu, Radiative corrections to the Yukawa coupling constants in two Higgs doublet models, Phys. Lett. B 731 (2014) 27 [arXiv:1401.0515] [INSPIRE].

[26] M. Krause, R. Lorenz, M. Mühlleitner, R. Santos and H. Ziesche, Gauge-independent Renormalization of the 2-Higgs-Doublet Model, JHEP 09 (2016) 143 [arXiv:1605.04853] [INSPIRE].

[27] A. Denner, L. Jenniches, J.-N. Lang and C. Sturm, Gauge-independent $\overline{M S}$ renormalization in the 2HDM, JHEP 09 (2016) 115 [arXiv:1607.07352] [INSPIRE].

[28] A. Denner, J.-N. Lang and S. Uccirati, NLO electroweak corrections in extended Higgs Sectors with RECOLA2, JHEP 07 (2017) 087 [arXiv:1705.06053] [INSPIRE].

[29] L. Altenkamp, S. Dittmaier and H. Rzehak, Renormalization schemes for the Two-Higgs-Doublet Model and applications to $h \rightarrow W W / Z Z \rightarrow 4$ fermions, JHEP 09 (2017) 134 [arXiv: 1704.02645] [INSPIRE].

[30] S. Kanemura, M. Kikuchi and K. Yagyu, Fingerprinting the extended Higgs sector using one-loop corrected Higgs boson couplings and future precision measurements, Nucl. Phys. B 896 (2015) 80 [arXiv:1502.07716] [INSPIRE].

[31] J. Fleischer and F. Jegerlehner, Radiative corrections to Higgs decays in the extended Weinberg-Salam Model, Phys. Rev. D 23 (1981) 2001 [INSPIRE].

[32] D. Binosi, Electroweak pinch technique to all orders, J. Phys. G 30 (2004) 1021 [hep-ph/0401182] [INSPIRE].

[33] A. Denner, G. Weiglein and S. Dittmaier, Gauge invariance of Green functions: Background field method versus pinch technique, Phys. Lett. B 333 (1994) 420 [hep-ph/9406204] [INSPIRE].

[34] A. Denner, G. Weiglein and S. Dittmaier, Application of the background field method to the electroweak standard model, Nucl. Phys. B 440 (1995) 95 [hep-ph/9410338] [InSPIRE].

[35] S. Kanemura, M. Kikuchi and K. Yagyu, Radiative corrections to the Higgs boson couplings in the model with an additional real singlet scalar field, Nucl. Phys. B 907 (2016) 286 [arXiv: 1511.06211] [INSPIRE].

[36] F. Bojarski, G. Chalons, D. Lopez-Val and T. Robens, Heavy to light Higgs boson decays at NLO in the Singlet Extension of the Standard Model, JHEP 02 (2016) 147 [arXiv: 1511.08120] [INSPIRE].

[37] L. Altenkamp, M. Boggia and S. Dittmaier, Precision calculations for $h \rightarrow W W / Z Z \rightarrow 4$ fermions in a Singlet Extension of the Standard Model with Prophecy 4 , JHEP 04 (2018) 062 [arXiv: 1801.07291] [INSPIRE].

[38] K.I. Aoki, Z. Hioki, M. Konuma, R. Kawabe and T. Muta, Electroweak Theory. Framework of On-Shell Renormalization and Study of Higher-Order Effects, Prog. Theor. Phys. Suppl. 73 (1982) 1 [INSPIRE].

[39] S. Actis, A. Ferroglia, M. Passera and G. Passarino, Two-loop renormalization in the Standard Model. Part I: Prolegomena, Nucl. Phys. B 777 (2007) 1 [hep-ph/0612122] [INSPIRE]. 
[40] G.M. Pruna and T. Robens, Higgs singlet extension parameter space in the light of the LHC discovery, Phys. Rev. D 88 (2013) 115012 [arXiv:1303.1150] [InSPIRE].

[41] L. Altenkamp, S. Dittmaier and H. Rzehak, Precision calculations for $h \rightarrow W W / Z Z \rightarrow 4$ fermions in the Two-Higgs-Doublet Model with Prophecy f, JHEP 03 (2018) 110 [arXiv: 1710.07598] [INSPIRE].

[42] A. Denner, S. Dittmaier, M. Roth and L.H. Wieders, Electroweak corrections to charged-current $e^{+} e^{-} \rightarrow 4$ fermion processes: Technical details and further results, Nucl. Phys. B 724 (2005) 247 [Erratum ibid. B 854 (2012) 504] [hep-ph/0505042] [INSPIRE].

[43] A. Denner and S. Dittmaier, The complex-mass scheme for perturbative calculations with unstable particles, Nucl. Phys. Proc. Suppl. 160 (2006) 22 [hep-ph/0605312] [INSPIRE].

[44] G. 't Hooft and M.J.G. Veltman, Scalar One Loop Integrals, Nucl. Phys. B 153 (1979) 365 [INSPIRE].

[45] G. 't Hooft, Renormalizable Lagrangians for Massive Yang-Mills Fields, Nucl. Phys. B 35 (1971) 167 [INSPIRE].

[46] G. 't Hooft, Renormalization of Massless Yang-Mills Fields, Nucl. Phys. B 33 (1971) 173 [INSPIRE].

[47] B.W. Lee and J. Zinn-Justin, Spontaneously Broken Gauge Symmetries. 1. Preliminaries, Phys. Rev. D 5 (1972) 3121 [INSPIRE].

[48] B.W. Lee and J. Zinn-Justin, Spontaneously broken gauge symmetries. 2. Perturbation theory and renormalization, Phys. Rev. D 5 (1972) 3137 [Erratum ibid. D 8 (1973) 4654] [INSPIRE].

[49] B.W. Lee and J. Zinn-Justin, Spontaneously broken gauge symmetries. 3. Equivalence, Phys. Rev. D 5 (1972) 3155 [INSPIRE].

[50] B.W. Lee and J. Zinn-Justin, Spontaneously Broken Gauge Symmetries. 4. General Gauge Formulation, Phys. Rev. D 7 (1973) 1049 [INSPIRE].

[51] B.W. Lee, Renormalization of gauge theories: Unbroken and broken, Phys. Rev. D 9 (1974) 933 [INSPIRE].

[52] M. Böhm, H. Spiesberger and W. Hollik, On the One-Loop Renormalization of the Electroweak Standard Model and Its Application to Leptonic Processes, Fortsch. Phys. 34 (1986) 687 [INSPIRE].

[53] P. Gambino and P.A. Grassi, The Nielsen identities of the SM and the definition of mass, Phys. Rev. D 62 (2000) 076002 [hep-ph/9907254] [INSPIRE].

[54] L.F. Abbott, The Background Field Method Beyond One Loop, Nucl. Phys. B 185 (1981) 189 [INSPIRE].

[55] S. Weinberg, The quantum theory of fields. Vol. 2: Modern applications, Cambridge University Press, (2013).

[56] M. Böhm, A. Denner and H. Joos, Gauge theories of the strong and electroweak interaction, Teubner, (2001).

[57] A. Bredenstein, A. Denner, S. Dittmaier and M.M. Weber, Precise predictions for the Higgs-boson decay $H \rightarrow W W / Z Z \rightarrow 4$ leptons, Phys. Rev. D 74 (2006) 013004 [hep-ph/0604011] [INSPIRE].

[58] A. Bredenstein, A. Denner, S. Dittmaier and M.M. Weber, Radiative corrections to the semileptonic and hadronic Higgs-boson decays $H \rightarrow W W / Z Z \rightarrow 4$ fermions, JHEP 02 (2007) 080 [hep-ph/0611234] [INSPIRE]. 
[59] A. Denner, S. Dittmaier, S. Kallweit and A. Mück, HAWK 2.0: A Monte Carlo program for Higgs production in vector-boson fusion and Higgs strahlung at hadron colliders, Comput. Phys. Commun. 195 (2015) 161 [arXiv:1412.5390] [INSPIRE].

[60] A. Denner et al., Higgs production and decay with a fourth standard-model-like fermion generation, Eur. Phys. J. C 72 (2012) 1992 [arXiv:1111.6395] [INSPIRE].

[61] M. Ciccolini, A. Denner and S. Dittmaier, Strong and electroweak corrections to the production of Higgs +2 jets via weak interactions at the LHC, Phys. Rev. Lett. 99 (2007) 161803 [arXiv: 0707.0381 ] [INSPIRE].

[62] M. Ciccolini, A. Denner and S. Dittmaier, Electroweak and QCD corrections to Higgs production via vector-boson fusion at the LHC, Phys. Rev. D 77 (2008) 013002 [arXiv: 0710.4749] [INSPIRE].

[63] A. Denner, S. Dittmaier, S. Kallweit and A. Mück, Electroweak corrections to Higgs-strahlung off $W / Z$ bosons at the Tevatron and the LHC with HAWK, JHEP 03 (2012) 075 [arXiv: 1112.5142] [INSPIRE].

[64] S. Actis, A. Denner, L. Hofer, J.-N. Lang, A. Scharf and S. Uccirati, RECOLA: REcursive Computation of One-Loop Amplitudes, Comput. Phys. Commun. 214 (2017) 140 [arXiv: 1605.01090] [INSPIRE].

[65] A. Denner, J.-N. Lang and S. Uccirati, RECOLA2: REcursive Computation of One-Loop Amplitudes 2, Comput. Phys. Commun. 224 (2018) 346 [arXiv:1711.07388] [InSPIRE].

[66] CMS collaboration, Search for the standard model Higgs boson produced in association with a W or a $Z$ boson and decaying to bottom quarks, Phys. Rev. D 89 (2014) 012003 [arXiv: 1310.3687] [INSPIRE].

[67] M. Cacciari, G.P. Salam and G. Soyez, The anti- $k_{t}$ jet clustering algorithm, JHEP 04 (2008) 063 [arXiv: 0802.1189] [INSPIRE].

[68] LHC Higgs Cross Section Working Group collaboration, D. de Florian et al., Handbook of LHC Higgs Cross Sections: 4. Deciphering the Nature of the Higgs Sector, arXiv: 1610.07922 [INSPIRE].

[69] A. Denner, S. Dittmaier and G. Weiglein, Green functions from a gauge invariant effective action for the electroweak standard model, Nucl. Phys. Proc. Suppl. B 37 (1994) 87 [hep-ph/9406400] [INSPIRE]. 